\title{
Skyrme pseudopotentials at next-to-next-to-leading order Construction of local densities and first symmetry-breaking calculations.
}

\author{
Wouter Ryssens* \\ Institut d'Astronomie et d'Astrophysique, Université Libre de Bruxelles, \\ Campus de la Plaine CP 226, BE-1050 Brussels, Belgium \\ Center for Theoretical Physics, Sloane Physics Laboratory, Yale University, New Haven, USA and \\ Université de Lyon, Université Claude Bernard Lyon 1, \\ CNRS, IP2I Lyon / IN2P3, UMR 5822, F-69622, Villeurbanne, France \\ Michael Bender \\ Université de Lyon, Université Claude Bernard Lyon 1, \\ CNRS, IP2I Lyon / IN2P3, UMR 5822, F-69622, Villeurbanne, France
}

(Dated: 30 June 2021)

\begin{abstract}
There is an ongoing quest to improve on the spectroscopic quality of nuclear energy density functionals (EDFs) of the Skyrme type through extensions of its traditional form. One direction for such activities is the inclusion of terms of higher order in gradients in the EDF. We report on exploratory symmetry-breaking calculations performed for an extension of the Skyrme EDF that includes central terms with four gradients at next-to-next-to-leading order (N2LO) and for which the high-quality parametrization SN2LO1 has been constructed recently [P. Becker et al, Phys. Rev. C 96, 044330 (2017)]. Up to now, the investigation of such functionals with higherorder terms was limited to infinite matter and spherically symmetric configurations of singly- and doubly-magic nuclei. We address here nuclei and phenomena that require us to consider axial and non-axial deformation, both for reflection-symmetric and also reflection-asymmetric shapes, as well as the breaking of time-reversal invariance. Achieving these calculations demanded a number of formal developments. These all resulted from the formulation of the N2LO EDF requiring the introduction of new local densities with additional gradients that are not present in the EDF at NLO. Their choice is not unique, but can differ in the way the gradients are coupled. While designing a numerical implementation of N2LO EDFs in Cartesian 3d coordinate-space representation, we have developed a novel definition and a new unifying notation for normal and pair densities that contain gradients at arbitrary order. Besides having mnemonic advantages, the new notation allows for the easy identification of redundancies and reducibilities in a given set of local densities, and the new definition makes it straightforward to construct densities that automatically adopt the symmetries of the many-body state they are constructed from. The resulting scheme resolves several issues with some of the choices that have been made for local densities in the past, in particular when breaking time-reversal symmetry. Guided by general practical considerations, we propose an alternative form of the N2LO contribution to the Skyrme EDF that is built from a different set of densities. It has exactly the same physics content, but is much more efficient to handle in formal discussions and, compared to the original formulation, leads to a substantial reduction of computational cost and memory requirements in deformed codes. As representative examples for the performance of SN2LO1, we have chosen the ground states of even-even $\mathrm{Kr}$ and $\mathrm{Nd}$ isotopes, the fission barrier of ${ }^{240} \mathrm{Pu}$, as well as the superdeformed rotational band of ${ }^{194} \mathrm{Hg}$. Overall, for the nuclei and phenomena studied here, the SN2LO1 parametrization does not yet present a systematic improvement over standard NLO parametrizations. This finding calls for improved fit protocols that better discriminate between NLO and N2LO terms and better exploit the unique features of the additional degrees of freedom offered by the latter.
\end{abstract}

\section{INTRODUCTION}

Methods employing nuclear energy density functionals (EDFs) have found wide-spread use in the nuclear physics community, providing the means to study both nuclear ground and excited states across the entire nuclear chart at a manageable numerical cost [1]. Functionals based on the non-relativistic zero-range Skyrme pseudopotential [1, 2] are arguably the most

\footnotetext{
* wryssens@ulb.ac.be

† bender@ip2i.in2p3.fr
}

widely used, and the description of bulk properties, such as the overall trends of nuclear binding energies and charge radii across the nuclear chart, can be pushed very far [3].

Despite this, there are inherent limitations to the standard form of the Skyrme functional. While generally on par with other types of functionals, the spectroscopic properties of Skyrme EDFs still leave much to be desired: examples include the description of the ground states of odd-mass nuclei [4] and the reproduction of shell gaps in the superheavy region [5]. A series of systematic studies [6 -8] led the UNEDF-Scidac collaboration to the conclusion that the spectroscopic qualities of the 
traditional Skyrme functional can no longer be improved in systematic fashion by changing the optimization procedure. For further advancements, one has to look at extensions of its traditional form instead.

Several possible avenues have been proposed in the literature: the addition of tensor terms to the Skyrme pseudopotential has been systematically explored in Refs. 9 13]. Another direction is the introduction of additional density dependencies for the coupling constants, both for terms without [14, 15] and with gradients [16 18]. We also mention the construction of Skyrme functionals that are strictly generated by a density-independent pseudopotential including threeand four-body terms with gradients that aim at spuriosity-free multi-reference calculations [19, 20].

The approach we will explore here is based on original work by the Jyväskylä group 21 24]. They proposed to revisit the possibility of adding terms with increasing number of gradients to the Skyrme EDF, some of which were in fact already considered in the original papers by Skyrme 25 28]. This type of extension can be made in a systematic fashion by studying all possible terms that can be added up to a given number of gradients: for a maximum of $2 \ell$ gradients, the resulting EDF is labelled N $\ell L O$. The traditional Skyrme pseudopotential, which includes terms up to two gradients, is labelled NLO, while Refs. 21 24 explored the possibilities of N3LO EDFs, including up to six gradients. Carlsson et al [21] formulated the most general bilinear form of such EDF that is compatible with the usual symmetry requirements, whereas Raimondi et al constructed the most general contact two-body pseudopotential with up to six gradients 24]. Both approaches yield an EDF of the same form, but the one obtained from the latter has a smaller number of independent coupling constants, see Appendix B, All of these developments were formulated in terms of gradients coupled to spherical tensors, which is well suited for a numerical implementation in spherical symmetry as the one documented in Ref. [22]. Using this code and a new strategy for density matrix expansion in terms of gradients instead of density dependencies, the authors of Ref. [23] demonstrated that the physics of finite-range interactions, including that of nonlocal exchange potentials, can be successfully mapped onto a Skyrme EDF in a systematically improvable manner when increasing the order in gradients. No other parameterization of a higher-order Skyrme EDF has been published by this group, though.

The further development of N $\ell$ LO EDFs was taken up by a collaboration of researchers from Lyon, Valencia, and York 29 37], who opted for a formulation in terms of Cartesian tensors as traditionally employed for the standard NLO EDF, and who limited themselves so far to the subset of pseudopotential-generated terms that are invariant under local gauge transformations. These terms are the ones that contribute to the equation of state of homogeneous infinite nuclear matter, and their influence on the latter was studied in detail in Refs. 29 34].
Again, it was found that the properties of nuclear matter obtained with various other approaches and interactions can be successfully mapped onto a Skyrme EDF when increasing the order in gradients. Adjusting parameters to infinite matter, however, is not sufficient to obtain a predictive parametrization for calculations of finite nuclei.

In the next step, this group constructed a spherical mean-field code that can handle higher-order terms in the Skyrme EDF [35, 36], and which has been subsequently used for the adjustment of the parameters of a Skyrme EDF augmented by central N2LO terms to the properties of finite (magic) nuclei 35 37. Named SN2LO1, this parameterization was adjusted with a protocol that is very similar to the one used for the adjustment of the SLy5 38, 39] and SLy5* 40] parametrizations of the NLO EDF. Like SLy5*, SN2LO1 has been fitted with additional stability constraints imposed via linearresponse calculations $34-36$. For masses and radii of spherical nuclei, the overall performance of these parametrizations is comparable. There is, however, the possibility that other observables and other nuclei that are usually not considered in a fit protocol might be more sensitive to the new terms, and that such information can be used to adjust N2LO EDFs that systematically outperform standard functionals.

For these reasons, there is an interest to extend calculations with the N2LO EDF to non-spherical nuclei. On general grounds, it is important to have a possibility to calculate observables of more complex nuclei that can inform future fits, and more specifically it will be instructive to benchmark the performance of SN2LO1 for deformed and rotating nuclei. For this reason, we study here for the first time the properties of deformed nuclei using an N2LO EDF. To this end, the N2LO EDF has been implemented into a 3d code in coordinate-space representation [41, 42], that allows for the exploration of numerous shape degrees of freedom including non-axial and non-reflection-symmetric ones, and finite angular momentum. As representative examples, we will discuss deformation properties of eveneven krypton and neodymium isotopes, the fission barrier of ${ }^{240} \mathrm{Pu}$, and the superdeformed rotational band of ${ }^{194} \mathrm{Hg}$. The latter also serves as a verification that time-reversal breaking calculations using the SN2LO1 parametrization are stable with respect to unphysical finite-size instabilities that plague many older Skyrme functionals at NLO [11, 43].

Designing the implementation of the additional terms into our numerical codes prompted us to examine in some detail the formulation of the N2LO terms in the Skyrme $\mathrm{EDF}$, and in particular the possible definitions of the local densities used in its construction. There are seven real normal densities and the same number of complex pair densities needed to define the most general Skyrme EDF at NLO [4], which are all well established in the literature. Going to higher-order EDFs requires one to introduce additional local densities at each order [21, 34]. 
There are several possible choices for their definition that are related to one another through the recoupling of gradient operators. These choices are not without consequences. On a formal and practical level, it is highly desirable to be able to split the EDF into parts built out of time-even and time-odd densities as it is the case for the traditional choice of densities entering the Skyrme EDF at NLO. Also, the NLO functional is traditionally formulated through local densities that also enter the expectation values of frequently-used onebody operators. For the densities that are specific to the EDF at N2LO and higher orders there is no such guidance anymore, and some of the definitions used in the literature lead to tensor densities whose components do not all transform in the same way under time-reversal. It is also not automatically guaranteed that the higher-order densities are linearly independent from one another. On a practical level, certain choices imply a larger computational burden than others. While designing an almost symmetry-unrestricted $3 \mathrm{~d}$ numerical implementation of N2LO EDFs in coordinate-space representation, we have developed a unifying notation for mean-field densities that allows for a much clearer discussion of the relevant degrees of freedom and makes it easier to identify redundancies in the set of local densities. Guided by general practical considerations, we propose an alternative version of the form of the N2LO EDF of Ref. 36] that is equivalent, but much more efficient to employ both in formal discussions and numerical implementations.

This paper is organized as follows: in Section [I] we present formal aspects of the past strategy to formulate the Skyrme EDF of Ref. [36] at N2LO just mentioned and point to some of its formal and computational inefficiencies, all of which can be expected to aggravate when setting up further extensions of the energy functional in the future. In Section III we then propose a novel scheme for a universal and systematic notation of local normal and pair densities and currents at an arbitrary order of gradients, and formulate a strategy to define local densities in a systematic manner that allows for easy identification of numerically efficient choices for a set of non-redundant and irreducible densities and the easy and transparent transformation between different such choices. In Sec. IV] we then apply the new definitions and notations to reformulate the N2LO Skyrme EDF of Ref. [36]. Finally, in Sec. $\mathrm{V}$ we report on exploratory calculations for deformed and rotating nuclei with SN2LO1 and compare with results obtained with similarly fitted parametrizations of the traditional NLO EDF. Section VI summarizes the main results of the paper, and appendices provide further technical and details concerning the properties of local densities and our implementation of the Skyrme EDF as needed for SN2LO1.

\section{THE N2LO ENERGY DENSITY FUNCTIONAL}

\section{A. The form of the functional}

The general form of the functional discussed here consists of five terms [1],

$$
E_{\mathrm{tot}}=E_{\mathrm{kin}}+E_{\mathrm{Sk}}+E_{\mathrm{Coul}}+E_{\mathrm{cm}}+E_{\mathrm{pair}},
$$

which correspond to the kinetic energy, the Skyrme EDF modeling of the strong interaction between the nucleons in the particle-hole channel, the Coulomb energy resulting from the electromagnetic repulsion between protons, a center-of-mass correction and a pairing term, respectively. In what follows, we will mainly address the second term, the Skyrme part, and we refer the reader to Ref. [45] for details on the treatment of the other terms.

Following the developments reported in Refs. [34 36], we consider here the Skyrme EDF as generated by a density-dependent, effective two-body pseudopotential $\hat{V}_{\text {Sk }}$ that consists of a central (C), spin-orbit (SO) and a (central) density-dependent (DD) part

$$
\hat{V}_{\mathrm{Sk}}=\hat{V}_{\mathrm{LO}}^{\mathrm{C}}+\hat{V}_{\mathrm{NLO}}^{\mathrm{C}}+\hat{V}_{\mathrm{N} 2 \mathrm{LO}}^{\mathrm{C}}+\hat{V}_{\mathrm{NLO}}^{\mathrm{SO}}+\hat{V}_{\mathrm{LO}}^{\mathrm{DD}} .
$$

Like these earlier references, we use a notation where the subscript refers to the number of gradients in the generators. Leading-order (LO) terms do not contain gradients, next-to-leading-order (NLO) terms contain two gradients, and next-to-next-to-leading order (N2LO) terms contain four gradients, and so on. The order of these terms refers to their degree of computational complexity and numerical cost, but does by no means represent a hierarchy of increasingly refined approximations to the nucleon-nucleon interaction such as the orders of an effective field theory would do.

The widely-used standard form of the Skyrme EDF combines terms up to NLO for central and spin-orbit terms, sometimes augmented also by a tensor interaction up to NLO [9 11], with a density-dependent (DD) term at LO. We consider here only its extension to N2LO for the central part of the pseudopotential, keeping the standard form for all other terms, including the other contributions to Eq. (1). Only for this particular extension do high-quality fits exist [36, 37]. As done in those references, we adopt only the N2LO terms that are invariant under local gauge transformations. We refer the reader to Ref. [36] for a detailed discussion of the form of the individual terms in Eq. (2).

The Skyrme part of the functional generated by the pseudopotential of Eq. (2) takes the generic form

$$
\begin{aligned}
& E_{\mathrm{Sk}}^{\mathrm{N} 2 \mathrm{LO}}=\int d^{3} r\left[\mathcal{E}^{(0)}(\mathbf{r})+\mathcal{E}^{(2)}(\mathbf{r})+\mathcal{E}^{(4)}(\mathbf{r})\right] \\
& \mathcal{E}^{(i)}(\mathbf{r})=\sum_{t=0,1}\left[\mathcal{E}_{t, \mathrm{e}}^{(i)}(\mathbf{r})+\mathcal{E}_{t, \mathrm{o}}^{(i)}(\mathbf{r})\right] \quad \text { for } i=0,2,4
\end{aligned}
$$


where $t=0,1$ labels terms composed of isoscalar and isovector densities, respectively. Except for the contribution of the density-dependent interaction, all terms in Eq. (3) are bilinear in some local normal onebody densities calculated from an auxiliary many-body state that is either a Slater determinant or a Bogoliubov quasiparticle vacuum. The superscripts (0), (2), and (4) indicate the order of the terms in terms of derivatives, whereas the subscripts e and o indicate whether the terms are constructed out of time-even or time-odd local densities.

For complete expressions of the LO and NLO energy densities, we refer the reader to Ref. [46]. Leaving the definition of the local densities to the next section, we specify here the full N2LO energy density in the notation of Ref. 36]

$$
\begin{aligned}
& \mathcal{E}_{t}^{(4)}(\mathbf{r})= C_{t}^{(4) \Delta \rho}\left[\Delta \rho_{t}(\mathbf{r})\right]^{2}+C_{t}^{(4) \Delta s}\left[\Delta \mathbf{s}_{t}(\mathbf{r})\right]^{2} \\
&+C_{t}^{(4) M \rho}\left\{\rho_{t}(\mathbf{r}) Q_{t}(\mathbf{r})+\tau_{t}^{2}(\mathbf{r})+2 \sum_{\mu \nu} \tau_{t, \mu \nu}(\mathbf{r}) \tau_{t, \mu \nu}(\mathbf{r})-2 \sum_{\mu \nu} \tau_{t, \mu \nu}(\mathbf{r})\left[\nabla_{\mu} \nabla_{\nu} \rho_{t}(\mathbf{r})\right]\right. \\
& \\
&\left.-\left[\nabla \cdot \mathbf{j}_{t}(\mathbf{r})\right]^{2}-4 \mathbf{j}_{t}(\mathbf{r}) \cdot \mathbf{\Pi}_{t}(\mathbf{r})\right\} \\
&+C_{t}^{(4) M \mathbf{s}}\left\{\mathbf{s}_{t}(\mathbf{r}) \cdot \mathbf{S}_{t}(\mathbf{r})+\mathbf{T}_{t}^{2}(\mathbf{r})+2 \sum_{\mu \nu \kappa} K_{t, \mu \nu \kappa}(\mathbf{r}) K_{t, \mu \nu \kappa}(\mathbf{r})-2 \sum_{\mu \nu \kappa} K_{t, \mu \nu \kappa}(\mathbf{r})\left[\nabla_{\mu} \nabla_{\nu} s_{t, \kappa}(\mathbf{r})\right]\right. \\
&\left.-\sum_{\nu}\left[\sum_{\mu} \nabla_{\mu} J_{t, \mu \nu}(\mathbf{r})\right]\left[\sum_{\kappa} \nabla_{\kappa} J_{t, \kappa \nu}(\mathbf{r})\right]-4 \sum_{\mu \nu} J_{t, \mu \nu}(\mathbf{r}) V_{t, \mu \nu}(\mathbf{r})\right\}
\end{aligned}
$$

where the four $C_{t}^{(4)}$ are coupling constants. As per usual, densities that are Cartesian tensors of rank 1 are written as vectors in boldface, and their contractions through inner and outer vector products. For higher-rank tensors, contractions are explicitly written out as summation over cartesian components indicated by greek indices. Besides the well-known densities that the standard NLO Skyrme EDF is constructed from, the energy density at N2LO additionally depends on the densities $Q_{t}(\mathbf{r})$, $\tau_{t, \mu \nu}(\mathbf{r}), \boldsymbol{\Pi}_{t}(\mathbf{r}), \mathbf{S}_{t}(\mathbf{r}), K_{t, \mu \nu \kappa}(\mathbf{r})$, and $V_{t, \mu \nu}(\mathbf{r})$ that will be defined in the next subsection. For reasons explained in Sec. II B 5, we do not attempt to separate the timeeven and time-odd parts of the energy density (5) but postpone this to its alternative form given in Sec. IVB.

\section{B. Traditional representation of densities}

\section{The one-body density matrix}

Depending on the treatment or not of pairing correlations, we will be dealing with either a single Slater determinant or a single Bogoliubov quasi-particle vacuum. We represent the auxiliary state $|\Phi\rangle$ from which the densities entering the EDF are constructed using a (for now) unspecified basis of orthonormal single-particle wave functions $\Psi_{k}(\mathbf{r})$

$$
\begin{aligned}
\Psi_{k}(\mathbf{r}) & =\sum_{\sigma= \pm} \sum_{q=p, n} \psi_{k}(\mathbf{r}, \sigma, q) \chi_{\sigma} \xi_{q} \\
& =\sum_{\sigma= \pm} \sum_{q=p, n}\left\langle\mathbf{r}, \sigma, q \mid \Psi_{k}\right\rangle \chi_{\sigma} \xi_{q}
\end{aligned}
$$

$$
=\sum_{\sigma= \pm} \sum_{q=p, n}\left\langle-\left|\hat{a}_{\mathbf{r} \sigma q} \hat{a}_{k}^{\dagger}\right|-\right\rangle \chi_{\sigma} \xi_{q}
$$

which are two-component spinors in both spin and isospin space spanned by the unit vectors $\chi_{\sigma}$ and $\xi_{q}$, respectively. Throughout this paper, we will assume that protons and neutrons are not mixed on the level of single-particle states. This implies that the auxiliary many-body state $|\Phi\rangle$ is the direct product of separate product states for protons and neutrons, respectively, $|\Phi\rangle=\left|\Phi_{p}\right\rangle \otimes\left|\Phi_{n}\right\rangle$. In this case, the full one-body normal and anomalous density matrices for the nucleon species $q=n, p$ can both be split into a scalar and a vector in spin space [47, 48].

Contact pairing interactions as usually used in the context of the Skyrme EDF naturally lead to a local pairing energy density that can be formulated in terms of local pair densities. Because of the anticommutation of fermionic annihilation operators, the anomalous density matrix $\kappa_{q}\left(\mathbf{r} \sigma, \mathbf{r}^{\prime} \sigma^{\prime}\right)=\left\langle\Phi\left|\hat{a}_{\mathbf{r}^{\prime} \sigma^{\prime} q} \hat{a}_{\mathbf{r} \sigma q}\right| \Phi\right\rangle$ is skew-symmetric under coordinate exchange. For this reason, one cannot construct local pair densities out of it in the same manner as is done for normal densities. This issue is resolved by using the so-called "Russian" representation of pair density matrices $\tilde{\rho}_{q}\left(\mathbf{r} \sigma, \mathbf{r}^{\prime} \sigma^{\prime}\right)$ instead of anomalous density matrices $\kappa_{q}\left(\mathbf{r} \sigma, \mathbf{r}^{\prime} \sigma^{\prime}\right)$ [47 50]. The full normal and pair one-body density matrix in position and spin space are given by

$$
\begin{aligned}
\rho_{q}\left(\mathbf{r} \sigma, \mathbf{r}^{\prime} \sigma^{\prime}\right) & \equiv\left\langle\Phi\left|\hat{a}_{\mathbf{r}^{\prime} \sigma^{\prime} q}^{\dagger} \hat{a}_{\mathbf{r} \sigma q}\right| \Phi\right\rangle \\
& =\sum_{j k} \rho_{k j} \psi_{j}^{*}\left(\mathbf{r}^{\prime}, \sigma^{\prime}\right) \psi_{k}(\mathbf{r}, \sigma)
\end{aligned}
$$




$$
=\frac{1}{2} \rho_{q}\left(\mathbf{r}, \mathbf{r}^{\prime}\right) \delta_{\sigma \sigma^{\prime}}+\frac{1}{2} \mathbf{s}_{q}\left(\mathbf{r}, \mathbf{r}^{\prime}\right) \cdot\left\langle\sigma|\hat{\boldsymbol{\sigma}}| \sigma^{\prime}\right\rangle
$$

$$
\begin{aligned}
\tilde{\rho}_{q}\left(\mathbf{r} \sigma, \mathbf{r}^{\prime} \sigma^{\prime}\right) & \equiv-\sigma^{\prime}\left\langle\Phi\left|\hat{a}_{\mathbf{r}^{\prime}-\sigma^{\prime} q} \hat{a}_{\mathbf{r} \sigma q}\right| \Phi\right\rangle \\
& =-\sigma^{\prime} \sum_{j k} \kappa_{k j} \psi_{j}\left(\mathbf{r}^{\prime},-\sigma^{\prime}\right) \psi_{k}(\mathbf{r}, \sigma) \\
& =\frac{1}{2} \tilde{\rho}_{q}\left(\mathbf{r}, \mathbf{r}^{\prime}\right) \delta_{\sigma \sigma^{\prime}}+\frac{1}{2} \tilde{\mathbf{s}}_{q}\left(\mathbf{r}, \mathbf{r}^{\prime}\right) \cdot\left\langle\sigma|\hat{\boldsymbol{\sigma}}| \sigma^{\prime}\right\rangle
\end{aligned}
$$

where $\hat{\boldsymbol{\sigma}}$ is the Cartesian vector of Pauli spin matrices. The sums over $j$ and $k$ run over the single-particle states of the nucleon species $q$. The $\rho_{k j}$ and $\kappa_{k j}$ are the elements of the normal and anomalous density matrices in the single-particle basis spanned by the $\Psi_{j}(\mathbf{r})$

$$
\begin{aligned}
\rho_{k j} & \equiv\left\langle\Phi\left|\hat{a}_{j}^{\dagger} \hat{a}_{k}\right| \Phi\right\rangle \\
\kappa_{k j} & \equiv\left\langle\Phi\left|\hat{a}_{j} \hat{a}_{k}\right| \Phi\right\rangle
\end{aligned}
$$

The non-local density $\rho_{q}\left(\mathbf{r}, \mathbf{r}^{\prime}\right)$, the non-local spin density $\mathbf{s}_{q}\left(\mathbf{r}, \mathbf{r}^{\prime}\right)$, the non-local pair density $\tilde{\rho}_{q}\left(\mathbf{r}, \mathbf{r}^{\prime}\right)$ and the nonlocal spin pair density $\tilde{\mathbf{s}}_{q}\left(\mathbf{r}, \mathbf{r}^{\prime}\right)$ are defined as

$$
\begin{aligned}
\rho_{q}\left(\mathbf{r}, \mathbf{r}^{\prime}\right) & \equiv \sum_{\sigma} \rho_{q}\left(\mathbf{r} \sigma, \mathbf{r}^{\prime} \sigma\right), \\
s_{q, \kappa}\left(\mathbf{r}, \mathbf{r}^{\prime}\right) & \equiv \sum_{\sigma \sigma^{\prime}} \rho\left(\mathbf{r} \sigma, \mathbf{r}^{\prime} \sigma^{\prime}\right)\left\langle\sigma^{\prime}\left|\hat{\sigma}_{\kappa}\right| \sigma\right\rangle, \\
\tilde{\rho}_{q}\left(\mathbf{r}, \mathbf{r}^{\prime}\right) & \equiv \sum_{\sigma} \tilde{\rho}_{q}\left(\mathbf{r} \sigma, \mathbf{r}^{\prime} \sigma\right), \\
\tilde{s}_{q, \kappa}\left(\mathbf{r}, \mathbf{r}^{\prime}\right) & \equiv \sum_{\sigma \sigma^{\prime}} \tilde{\rho}_{q}\left(\mathbf{r} \sigma, \mathbf{r}^{\prime} \sigma^{\prime}\right)\left\langle\sigma^{\prime}\left|\hat{\sigma}_{\kappa}\right| \sigma\right\rangle .
\end{aligned}
$$

These non-local densities behave as follows under the exchange of $\mathbf{r} \leftrightarrow \mathbf{r}^{\prime}$ :

$$
\begin{array}{cc}
\rho_{q}\left(\mathbf{r}, \mathbf{r}^{\prime}\right)=+\rho_{q}^{*}\left(\mathbf{r}^{\prime}, \mathbf{r}\right), & \mathbf{s}_{q}\left(\mathbf{r}, \mathbf{r}^{\prime}\right)=+\mathbf{s}_{q}^{*}\left(\mathbf{r}^{\prime}, \mathbf{r}\right), \\
\tilde{\rho}_{q}\left(\mathbf{r}, \mathbf{r}^{\prime}\right)=+\tilde{\rho}_{q}\left(\mathbf{r}^{\prime}, \mathbf{r}\right), & \tilde{\mathbf{s}}_{q}\left(\mathbf{r}, \mathbf{r}^{\prime}\right)=-\tilde{\mathbf{s}}_{q}\left(\mathbf{r}^{\prime}, \mathbf{r}\right) .
\end{array}
$$

The density matrices associated with the time-reversed auxiliary many-body state $\check{T}|\Phi\rangle$ are related to those of the original state $|\Phi\rangle$ through [44]

$$
\begin{aligned}
& \rho_{q}^{T}\left(\mathbf{r} \sigma, \mathbf{r}^{\prime} \sigma^{\prime}\right)=\sigma \sigma^{\prime} \rho_{q}^{*}\left(\mathbf{r}-\sigma, \mathbf{r}^{\prime}-\sigma^{\prime}\right), \\
& \tilde{\rho}_{q}^{T}\left(\mathbf{r} \sigma, \mathbf{r}^{\prime} \sigma^{\prime}\right)=\sigma \sigma^{\prime} \tilde{\rho}_{q}^{*}\left(\mathbf{r}-\sigma, \mathbf{r}^{\prime}-\sigma^{\prime}\right) .
\end{aligned}
$$

These relations then provide the starting point to deduce the symmetry properties under time-reversal of the local densities that will be defined in what follows.

Inserting the expansion of $\rho_{q}\left(\mathbf{r} \sigma, \mathbf{r}^{\prime} \sigma^{\prime}\right)$ into singleparticle wave functions (7D) into the definition of the nonlocal normal densities, one finds

$$
\begin{gathered}
\rho_{q}\left(\mathbf{r}, \mathbf{r}^{\prime}\right)=\sum_{j k} \rho_{k j} \Psi_{j}^{\dagger}\left(\mathbf{r}^{\prime}\right) \Psi_{k}(\mathbf{r}), \\
s_{q, \kappa}\left(\mathbf{r}, \mathbf{r}^{\prime}\right)=\sum_{j k} \rho_{k j} \Psi_{j}^{\dagger}\left(\mathbf{r}^{\prime}\right) \hat{\sigma}_{\kappa} \Psi_{k}(\mathbf{r}),
\end{gathered}
$$

where the sum over single-particle orbits runs over those of the nucleon species $q$. Doing the same for the nonlocal pair densities, Eqs. (13) and (14), the latter can be redefined as

$$
\begin{gathered}
\tilde{\rho}_{q}\left(\mathbf{r}, \mathbf{r}^{\prime}\right) \equiv \sum_{j k} \kappa_{k j} \tilde{\varrho}_{j k}\left(\mathbf{r}, \mathbf{r}^{\prime}\right), \\
\tilde{s}_{q, \mu}\left(\mathbf{r}, \mathbf{r}^{\prime}\right) \equiv \sum_{j k} \kappa_{k j} \tilde{\varsigma}_{j k, \mu}\left(\mathbf{r}, \mathbf{r}^{\prime}\right),
\end{gathered}
$$

using the objects

$$
\begin{aligned}
\tilde{\varrho}_{j k}\left(\mathbf{r}, \mathbf{r}^{\prime}\right) & \equiv \sum_{\sigma} \sigma \psi_{j}\left(\mathbf{r}^{\prime}, \sigma\right) \psi_{k}(\mathbf{r},-\sigma), \\
\tilde{\varsigma}_{j k, \mu}\left(\mathbf{r}, \mathbf{r}^{\prime}\right) & \equiv \sum_{\sigma, \sigma^{\prime}} \sigma^{\prime} \psi_{j}\left(\mathbf{r}^{\prime}, \sigma^{\prime}\right) \psi_{k}(\mathbf{r}, \sigma)\left\langle-\sigma^{\prime}\left|\hat{\sigma}_{\mu}\right| \sigma\right\rangle .
\end{aligned}
$$

The first one of these, $\tilde{\varrho}_{j k}\left(\mathbf{r}, \mathbf{r}^{\prime}\right)$ is proportional to the two-body wave function of a spin-singlet state, $S=0$, with the third component of isospin $T_{3}= \pm 1$ and is therefore a member of an isospin-triplet, $T=1$. In contrast, the three Cartesian components of $\tilde{\varsigma}_{j k, \mu}\left(\mathbf{r}, \mathbf{r}^{\prime}\right)$ are linear combinations of the three possible two-body wave functions of a spin-triplet state, $S=1$, again with $T=1$, which gives an indication of the physics described by terms that contain these objects. This observation also explains the attractiveness of using the "Russian representation" instead of the traditional one based on $\kappa\left(\mathbf{r} \sigma, \mathbf{r}^{\prime} \sigma^{\prime}\right)$ that cannot be further broken down into objects with a physical interpretation.

These objects have the following symmetries under the simultaneous exchange of the single-particle states $j$ and $k$ and their positions

$$
\begin{aligned}
\tilde{\varrho}_{j k}\left(\mathbf{r}, \mathbf{r}^{\prime}\right) & =-\tilde{\varrho}_{k j}\left(\mathbf{r}^{\prime}, \mathbf{r}\right), \\
\tilde{\varsigma}_{j k, \mu}\left(\mathbf{r}, \mathbf{r}^{\prime}\right) & =+\tilde{\varsigma}_{k j, \mu}\left(\mathbf{r}^{\prime}, \mathbf{r}\right) .
\end{aligned}
$$

Using the phase convention $\psi_{k}^{T}(\mathbf{r}, \sigma)=-\sigma \psi_{k}(\mathbf{r},-\sigma)$, in the time-reversed auxiliary state these objects become

$$
\begin{aligned}
\tilde{\varrho}_{j k}^{T}\left(\mathbf{r}, \mathbf{r}^{\prime}\right) & =+\tilde{\varrho}_{k j}^{*}\left(\mathbf{r}, \mathbf{r}^{\prime}\right), \\
\tilde{\varsigma}_{j k, \mu}^{T}\left(\mathbf{r}, \mathbf{r}^{\prime}\right) & =-\tilde{\varsigma}_{k j, \mu}^{*}\left(\mathbf{r}, \mathbf{r}^{\prime}\right) .
\end{aligned}
$$

By the consecutive application of derivatives with respect to either $\mathbf{r}$ or $\mathbf{r}^{\prime}$ to the non-local normal and pair densities defined through Eqs. (11)-(14), setting $\mathbf{r}=\mathbf{r}^{\prime}$ afterwards, one can now construct a multitude of different local densities, which in turn can be used to build terms of a local energy density functional. We will introduce the local densities entering the energy densities of Eq. (3) order-by-order in the next sections.

A final remark is in order: we concern ourselves here chiefly with static self-consistent mean-field calculations. Our discussion will be limited to the normal and pair densities of a single auxiliary state, and does not extend to the more general calculation of transition (also called mixed) densities between different auxiliary states, $\left\langle\Phi^{\prime}\left|\hat{a}_{\mathbf{r}^{\prime} \sigma^{\prime} q^{\prime}}^{\dagger} \hat{a}_{\mathbf{r} \sigma q}\right| \Phi\right\rangle /\left\langle\Phi^{\prime} \mid \Phi\right\rangle,\left\langle\Phi^{\prime}\left|\hat{a}_{\mathbf{r}^{\prime} \sigma^{\prime} q^{\prime}}^{\dagger} \hat{a}_{\mathbf{r} \sigma q}^{\dagger}\right| \Phi\right\rangle /\left\langle\Phi^{\prime} \mid \Phi\right\rangle$ and 
$\left\langle\Phi^{\prime}\left|\hat{a}_{\mathbf{r}^{\prime} \sigma^{\prime} q^{\prime}} \hat{a}_{\mathbf{r} \sigma q}\right| \Phi\right\rangle /\left\langle\Phi^{\prime} \mid \Phi\right\rangle$. The calculation of these objects is required, for example, when restoring broken symmetries or when employing the generator coordinate method [1]. As these objects are in general complexvalued functions, the following discussion regarding the reality of specific densities has to be replaced by an analysis of the hermiticity of the corresponding densities, that is to say their symmetry under exchange of $|\Phi\rangle$ and $\left|\Phi^{\prime}\right\rangle$, which would lead to the same conclusions for preferable choices for the definition of local densities.

\section{LO: Local densities appearing in $\mathcal{E}^{(0)}$}

When discarding the possibility of proton-neutron mixing as done here, it is only possible to construct three different gradientless local densities at leading order. These are the normal local density $\rho_{q}(\mathbf{r})$, the normal spin density $\mathbf{s}_{q}(\mathbf{r})$, and the local pair density $\tilde{\rho}_{q}(\mathbf{r})$,

$$
\begin{aligned}
\rho_{q}(\mathbf{r}) & \left.\equiv \rho_{q}\left(\mathbf{r}, \mathbf{r}^{\prime}\right)\right|_{\mathbf{r}=\mathbf{r}^{\prime}}, \\
\mathbf{s}_{q}(\mathbf{r}) & \left.\equiv \mathbf{s}_{q}\left(\mathbf{r}, \mathbf{r}^{\prime}\right)\right|_{\mathbf{r}=\mathbf{r}^{\prime}}, \\
\tilde{\rho}_{q}(\mathbf{r}) & \left.\equiv \tilde{\rho}_{q}\left(\mathbf{r}, \mathbf{r}^{\prime}\right)\right|_{\mathbf{r}=\mathbf{r}^{\prime}}
\end{aligned}
$$

The local part of the spin pair density $\tilde{\mathbf{s}}_{q}(\mathbf{r})$ is identical to zero, which can be easily deduced from Eq. (16), and which explains the absence of a fourth local density 1 From Eq. (15) follows that both $\rho_{q}(\mathbf{r})$ and $\mathbf{s}_{q}(\mathbf{r})$ are realvalued functions, even if the matrix elements $\rho_{k j}$ and the single-particle wave functions are not. By contrast, $\tilde{\rho}_{q}(\mathbf{r})$ is in general a complex-valued spatial function.

Using Eq. (17) and Eq. (18), we can determine the local densities of the time-reversed auxiliary state

$$
\begin{gathered}
\rho_{q}^{T}(\mathbf{r})=+\rho_{q}(\mathbf{r}), \\
\mathbf{s}_{q}^{T}(\mathbf{r})=-\mathbf{s}_{q}(\mathbf{r}), \\
\tilde{\rho}_{q}^{T}(\mathbf{r})=+\tilde{\rho}_{q}^{*}(\mathbf{r}) .
\end{gathered}
$$

Hence $\rho_{q}(\mathbf{r})$ is time-even, whereas $\mathbf{s}_{q}(\mathbf{r})$ is time-odd. The pair density $\tilde{\rho}_{q}(\mathbf{r})$ in its entirety is neither time-even nor time-odd: its real part is time-even, while its imaginary part is time-odd.

\section{NLO: Local densities additionally appearing in $\mathcal{E}^{(2)}$}

By acting with either one or two derivatives on the non-local densities, we obtain five different normal and two different pair densities that enter the EDF at NLO. These normal local densities are given by

$$
\left.\tau_{q}(\mathbf{r}) \equiv \nabla \cdot \nabla^{\prime} \rho_{q}\left(\mathbf{r}, \mathbf{r}^{\prime}\right)\right|_{\mathbf{r}=\mathbf{r}^{\prime}},
$$

\footnotetext{
${ }^{1}$ We note, however, that a local isoscalar spin pair density appears naturally when also considering proton-neutron-mixing $T=0$ pairing correlations [44, 51].
}

$$
\begin{aligned}
\mathbf{j}_{q}(\mathbf{r}) & \equiv-\left.\frac{\mathrm{i}}{2}\left(\nabla-\nabla^{\prime}\right) \rho_{q}\left(\mathbf{r}, \mathbf{r}^{\prime}\right)\right|_{\mathbf{r}=\mathbf{r}^{\prime}}, \\
T_{q, \mu}(\mathbf{r}) & \left.\equiv \nabla \cdot \nabla^{\prime} s_{q, \mu}\left(\mathbf{r}, \mathbf{r}^{\prime}\right)\right|_{\mathbf{r}=\mathbf{r}^{\prime}}, \\
F_{q, \mu}(\mathbf{r}) & \left.\equiv \frac{1}{2} \sum_{\nu}\left[\nabla_{\mu} \nabla_{\nu}^{\prime}+\nabla_{\mu}^{\prime} \nabla_{\nu}\right] s_{q, \nu}\left(\mathbf{r}, \mathbf{r}^{\prime}\right)\right|_{\mathbf{r}=\mathbf{r}^{\prime}}, \\
J_{q, \mu \nu}(\mathbf{r}) & \equiv-\left.\frac{\mathrm{i}}{2}\left(\nabla_{\mu}-\nabla_{\mu}^{\prime}\right) s_{q, \nu}\left(\mathbf{r}, \mathbf{r}^{\prime}\right)\right|_{\mathbf{r}=\mathbf{r}^{\prime}},
\end{aligned}
$$

where $\mu, \nu, \kappa$ label the three cartesian directions of space. Three of these densities, which are $\tau_{q}(\mathbf{r})$, $\mathbf{T}_{q}(\mathbf{r})$, and $\mathbf{F}_{q}(\mathbf{r})$, are kinetic-type densities involving the successive application of two gradients acting on different coordinates, while the two others, that is $\mathbf{j}_{q}(\mathbf{r})$ and $J_{q, \mu \nu}(\mathbf{r})$, are of current type, involving the difference of two gradient operators acting on different coordinates. The density $\mathbf{F}_{q}(\mathbf{r})$ in general only appears for parametrizations that include explicit tensor forces, such as considered in Refs. [9 11], and does not play a role for the presently available parametrizations considering N2LO terms; we have included it here for completeness' sake. There are a few additional possible densities with two gradients, but they cannot be used to construct suitable scalar contributions to the energy density at this order [48, 52].

For the pair densities that can contribute to the pair EDF at NLO [19, 47], we write

$$
\begin{aligned}
\tilde{\tau}_{q}(\mathbf{r}) & \left.\equiv \boldsymbol{\nabla} \cdot \nabla^{\prime} \tilde{\rho}_{q}\left(\mathbf{r}, \mathbf{r}^{\prime}\right)\right|_{\mathbf{r}=\mathbf{r}^{\prime}}, \\
\tilde{J}_{q, \mu \nu}(\mathbf{r}) & \equiv-\left.\frac{\mathrm{i}}{2}\left(\nabla_{\mu}-\nabla_{\mu}^{\prime}\right) \tilde{s}_{q, \nu}\left(\mathbf{r}, \mathbf{r}^{\prime}\right)\right|_{\mathbf{r}=\mathbf{r}^{\prime}} .
\end{aligned}
$$

where we have one kinetic-type $\left(\tilde{\tau}_{q}\right)$ and one current-type pair density $\left(\tilde{J}_{q, \mu \nu}\right)$.

Together with the three gradientless densities, the seven densities presented in this section form the backbone of the many different forms of the Skyrme functional up to NLO found in the literature In fact, it can be shown that, in the absence of proton-neutron mixing, together with some of their derivatives these local densities are sufficient to express any local energy density with up to two gradients that is compatible with the usual symmetries of the nuclear Hamiltonian [44].

These traditional definitions have the advantage that all normal NLO densities are real-valued functions and are either time-even or time-odd:

$$
\begin{aligned}
\tau_{q}^{T}(\mathbf{r}) & =+\tau_{q}(\mathbf{r}), & \mathbf{j}_{q}^{T}(\mathbf{r}) & =-\mathbf{j}_{q}(\mathbf{r}), \\
\mathbf{T}_{q}^{T}(\mathbf{r}) & =-\mathbf{T}_{q}(\mathbf{r}), & J_{q, \mu \nu}^{T}(\mathbf{r}) & =+J_{q, \mu \nu}(\mathbf{r}),
\end{aligned}
$$

\footnotetext{
2 The pair densities contributing to the NLO pair functional are only rarely incorporated, notable exceptions being Refs. [19, 47] where the same coupling constants determine the particlehole and pairing parts of the EDF. Most applications of the Skyrme EDF, however, use an independent pairing EDF that is constructed only out of the LO pair densities such as the one of Eq. 148 that will be used here.
} 


$$
\mathbf{F}_{q}^{T}(\mathbf{r})=-\mathbf{F}_{q}(\mathbf{r})
$$

As at LO, the pair densities are neither time-even nor time-odd

$$
\tilde{\tau}_{q}^{T}(\mathbf{r})=+\tilde{\tau}_{q}^{*}(\mathbf{r}), \quad \tilde{J}_{q, \mu \nu}^{T}(\mathbf{r})=+\tilde{J}_{q, \mu \nu}^{*}(\mathbf{r}) .
$$

Again, their real parts are time-even, while the imaginary parts are time-odd.

\section{N2LO: Local densities additionally appearing in $\mathcal{E}^{(4)}$}

We are aware of two existing conventions in the literature for densities at N2LO: a scheme based on spherical tensors introduced in Ref. 21], and a scheme based on cartesian tensors, more analogous to the traditional densities of the NLO Skyrme functional, introduced first in Ref. [34] and amended in Ref. [36]. As we will discuss in what follows, these two schemes are but two out of many different, but equivalent, possibilities to define such densities. Of the two approaches, we will stay closer to the choice of Ref. [36], as the spherical tensors used in Ref. 21] are complicated to use when considering deformed nuclei.

The choice made in Ref. [36] consists of the following four local normal densities

$$
\begin{aligned}
Q_{q}(\mathbf{r}) & \left.\equiv \Delta \Delta^{\prime} \rho_{q}\left(\mathbf{r}, \mathbf{r}^{\prime}\right)\right|_{\mathbf{r}=\mathbf{r}^{\prime}} \\
\mathbf{S}_{q}(\mathbf{r}) & \left.\equiv \Delta \Delta^{\prime} \mathbf{s}_{q}\left(\mathbf{r}, \mathbf{r}^{\prime}\right)\right|_{\mathbf{r}=\mathbf{r}^{\prime}} \\
\Pi_{q, \mu}(\mathbf{r}) & \equiv-\left.\frac{i}{2}\left(\nabla_{\mu}-\nabla_{\mu}^{\prime}\right)\left(\boldsymbol{\nabla} \cdot \nabla^{\prime}\right) \rho_{q}\left(\mathbf{r}, \mathbf{r}^{\prime}\right)\right|_{\mathbf{r}=\mathbf{r}^{\prime}} \\
V_{q, \mu \nu}(\mathbf{r}) & \equiv-\left.\frac{i}{2}\left(\nabla_{\mu}-\nabla_{\mu}^{\prime}\right)\left(\boldsymbol{\nabla} \cdot \nabla^{\prime}\right) s_{q, \nu}\left(\mathbf{r}, \mathbf{r}^{\prime}\right)\right|_{\mathbf{r}=\mathbf{r}^{\prime}},
\end{aligned}
$$

which are all real spatial functions by construction. In addition to these four new normal densities, the authors of Ref. [36] define also the following kinetic-type normal tensor densities

$$
\begin{aligned}
\tau_{q, \mu \nu}(\mathbf{r}) & \left.\equiv \nabla_{\mu} \nabla_{\nu}^{\prime} \rho_{q}\left(\mathbf{r}, \mathbf{r}^{\prime}\right)\right|_{\mathbf{r}=\mathbf{r}^{\prime}}, \\
K_{q, \mu \nu \kappa}(\mathbf{r}) & \left.\equiv \nabla_{\mu} \nabla_{\nu}^{\prime} s_{q, \kappa}\left(\mathbf{r}, \mathbf{r}^{\prime}\right)\right|_{\mathbf{r}=\mathbf{r}^{\prime}},
\end{aligned}
$$

which are in general complex spatial functions. Note that not every component of either density is a complex number with non-zero real and imaginary parts: $\tau_{x x}(\mathbf{r})$, $\tau_{y y}(\mathbf{r})$ and $\tau_{z z}(\mathbf{r})$ are for instance all real functions as they make up the scalar part of the tensor. The densities $\tau_{q}(\mathbf{r}), \mathbf{T}_{q}(\mathbf{r})$ and $\mathbf{F}_{q}(\mathbf{r})$ that already entered $\mathcal{E}^{(2)}(\mathbf{r})$ are different tensor contractions of the full tensors $\tau_{q, \mu \nu}(\mathbf{r})$ and $K_{q, \mu \nu \kappa}(\mathbf{r})$ with the Kronecker symbol $\delta_{\lambda \chi}$. Although these densities only contain two gradients (not three or four), they only become relevant at the N2LO level since there are no other densities at the NLO level that they can be combined with to a form a scalar term in the energy density [52].

Even though they do not play a role in the functional of Ref. [36], we can construct the following pair densities by analogy

$$
\tilde{Q}_{q}(\mathbf{r})=\left.\Delta \Delta^{\prime} \tilde{\rho}_{q}\left(\mathbf{r}, \mathbf{r}^{\prime}\right)\right|_{\mathbf{r}=\mathbf{r}^{\prime}},
$$

$$
\begin{aligned}
\tilde{V}_{q, \mu \nu}(\mathbf{r}) & =-\left.\frac{\mathrm{i}}{2}\left(\nabla_{\mu}-\nabla_{\mu}^{\prime}\right)\left(\boldsymbol{\nabla} \cdot \nabla^{\prime}\right) \tilde{s}_{q, \nu}\left(\mathbf{r}, \mathbf{r}^{\prime}\right)\right|_{\mathbf{r}=\mathbf{r}^{\prime}} \\
\tilde{\tau}_{q, \mu \nu} & =\left.\nabla_{\mu} \nabla_{\nu}^{\prime} \tilde{\rho}_{q}\left(\mathbf{r}, \mathbf{r}^{\prime}\right)\right|_{\mathbf{r}=\mathbf{r}^{\prime}}
\end{aligned}
$$

Again, in the absence of proton-neutron mixing, it follows from Eq. (16) that the pair densities $\tilde{\mathbf{S}}_{q}(\mathbf{r})$ and $\tilde{K}_{q, \mu \nu \kappa}(\mathbf{r})$ that are the analogues of the normal densities defined in Eqs. (43) and (47) are zero, as is the pair current $\tilde{\Pi}_{q, \mu}(\mathbf{r})$ that is the analogue of Eq. (44).

Using Eq. (17), one can easily establish the following relations for the new normal densities

$$
\begin{aligned}
Q_{q}^{T}(\mathbf{r}) & =+Q_{q}(\mathbf{r}), & & \mathbf{S}_{q}^{T}(\mathbf{r})=-\mathbf{S}_{q}(\mathbf{r}), \\
V_{q, \mu \nu}^{T}(\mathbf{r}) & =+V_{q, \mu \nu}(\mathbf{r}), & \Pi_{q}^{T}(\mathbf{r}) & =-\Pi_{q}(\mathbf{r}), \\
\tau_{q, \mu \nu}^{T} & =+\tau_{q, \mu \nu}^{*}, & & K_{q, \mu \nu \kappa}^{T}=-K_{q, \mu \nu \kappa}^{*} .
\end{aligned}
$$

The new real densities $Q_{q}(\mathbf{r})$ and $V_{q, \mu \nu}(\mathbf{r})$ are both timeeven while $\mathbf{S}_{q}(\mathbf{r})$ and $\boldsymbol{\Pi}_{q}(\mathbf{r})$ are time-odd. By contrast, the complex densities $\tau_{q, \mu \nu}(\mathbf{r})$ and $K_{q, \mu \nu \kappa}(\mathbf{r})$ do not have a definite behavior under time-reversal: $\operatorname{Re}\left\{\tau_{q, \mu \nu}(\mathbf{r})\right\}$ and $\operatorname{Im}\left\{K_{q, \mu \nu \kappa}(\mathbf{r})\right\}$ are time-even, whereas $\operatorname{Im}\left\{\tau_{q, \mu \nu}(\mathbf{r})\right\}$ and $\operatorname{Re}\left\{K_{q, \mu \nu \kappa}(\mathbf{r})\right\}$ are time-odd. Ultimately, this is a consequence of the operator $\nabla_{\mu} \nabla_{\nu}^{\prime}$ that generates these densities in Eqs. (46) and (47) not being hermitian.

In similar fashion, we can use Eq. (18) for the pair densities and deduce

$$
\begin{gathered}
\tilde{Q}_{q}^{T}(\mathbf{r})=+\tilde{Q}^{*}(\mathbf{r}), \\
\tilde{V}_{q, \mu \nu}^{T}(\mathbf{r})=+\tilde{V}_{q, \mu \nu}^{*}(\mathbf{r}), \\
\tilde{\tau}_{q, \mu \nu}^{T}(\mathbf{r})=+\tilde{\tau}_{q, \mu \nu}^{*}(\mathbf{r}) .
\end{gathered}
$$

As is the case for the pair densities entering the LO and NLO functional, all of the new pair densities are complex functions, with real parts that are time even while the imaginary parts are time odd.

\section{Problems with this scheme}

There are three main reasons to devise a better accounting of the mean-field densities. The first one is that one cannot keep up in a reasonable way the letter-naming scheme of Ref. [36]: at N2LO level one has to introduce four new normal densities (and two pair densities) in addition to higher-rank tensor versions of those already present at NLO. Combined with those needed for the leading order Skyrme functional, the full N2LO functional is formulated in terms of eleven distinct local densities. Each of these leads to a distinct potential for which also a symbol has to be chosen. At higher orders this number will be further inflated such that at some point one will run out of letters of the roman and greek alphabets that can be unambiguously used to represent these densities and the corresponding potentials, not to mention the increasing mnemonic difficulties to associate these symbols with the object that they represent. A more extensible notation is desired, 
which preferably links the underlying operator structure to the symbol used for a given density.

The second is a problem of efficiency: at any given order in gradients there are many ways to recouple gradient operators that act on the non-local densities. For example: the imaginary part of $\tau_{q, \mu \nu}(\mathbf{r})$ can be rewritten as a linear combination of external gradients acting on the current density $\mathbf{j}_{q}(\mathbf{r})$ [53]:

$$
\operatorname{Im}\left\{\tau_{q, \mu \nu}(\mathbf{r})\right\}=\frac{1}{2}\left[\nabla_{\mu} j_{q, \nu}(\mathbf{r})-\nabla_{\nu} j_{q, \mu}(\mathbf{r})\right] .
$$

This relation can be easily shown using Eq. 899, which will explained in the next subsection. We will call densities that can be rewritten as linear combinations of gradients of lower order densities reducible. Equation (57) demonstrates that $\operatorname{Im}\left\{\tau_{q \mu \nu}(\mathbf{r})\right\}$ is reducible: it can be completely eliminated from any formulation of the EDF in favor of terms involving gradients acting on the components of $\mathbf{j}_{q}(\mathbf{r})$.

Another example is the definition of an alternative density touched upon briefly in Ref. [35], which involves four gradients,

$$
\left.\mathcal{T}_{q}(\mathbf{r}) \equiv\left(\nabla^{\prime} \cdot \nabla\right)\left(\nabla^{\prime} \cdot \nabla\right) \rho_{q}\left(\mathbf{r}, \mathbf{r}^{\prime}\right)\right|_{\mathbf{r}=\mathbf{r}^{\prime}} .
$$

While $\mathcal{T}_{q}(\mathbf{r})$ is a valid local density that can be used to define an EDF, with the help of relations that will be explained in the next subsection one can show that it can be rewritten as a linear combination of the N2LO density $Q_{q}(\mathbf{r})$ defined in Eq. (48) that also contains four gradients and second derivatives of the kinetic tensor density $\tau_{q, \mu \nu}(\mathbf{r})$ that contains just two gradients

$$
\mathcal{T}_{q}(\mathbf{r})=Q_{q}(\mathbf{r})+\Delta \tau_{q}(\mathbf{r})-\sum_{\mu \nu} \nabla_{\mu} \nabla_{\nu} \tau_{q, \mu \nu}(\mathbf{r}) .
$$

It is clear that when choosing the local densities $Q_{q}(\mathbf{r})$, $\tau_{q, \mu \nu}(\mathbf{r})$, and $\tau_{q}(\mathbf{r})$ to express an EDF, one does not need to additionally consider $\mathcal{T}_{q}(\mathbf{r})$. 3

Because of the possibility of redundancies such as Eq. (59) and the existence of reducible densities, it is not trivial to choose a set of mean-field densities at arbitrary order in gradients. The problem can arrive in two different ways: either when generating the EDF from a pseudopotential (2), which necessitates to recouple the gradients in such a way that they can be expressed through suitable local densities [19, 24, 44, 54], or when setting up the EDF directly as a combination of densities that respects the usual symmetry requirements [13, 21, 52, 55. In either case one would like to allow for the most

\footnotetext{
${ }^{3}$ Note that $Q_{q}(\mathbf{r})$ and $\mathcal{T}_{q}(\mathbf{r})$ become equal in infinite homogeneous nuclear matter. Also, the integral over $\mathcal{T}_{q}(\mathbf{r})$ is always equal to the integral over $Q_{q}(\mathbf{r})$, although for systems with a surface they will look unalike when plotted. What will be different for such systems are integrals where either $\mathcal{T}_{q}(\mathbf{r})$ or $Q_{q}(\mathbf{r})$ multiplies another function or density, such as bilinear or trilinear terms in the EDF.
}

general EDF but without introducing extra overhead for the densities, neither formally in the multitude of symbols to define and memorize, nor numerically in the number of objects to be calculated. As will be sketched in Appendix E, at the NLO level this only concerns the kinetic densities. For these, however, it is known for long that using one or the other of the possible forms can lead to subtle differences when setting up semi-classical approximations.

While this problem is still somewhat limited at the N2LO level detailed here, the amount of possible redundancies rises markedly when going to higher orders. From general arguments about the symmetries of a local bilinear EDF whose terms contain in total six gradients, one can deduce that the construction of the additional central terms that emerge at N3LO will require two kinetic-type densities involving six derivatives, a scalar one and a pseudovector spin density, as well as two current-type densities involving five derivatives, one being a vector density, the other a rank-2 spin-currenttype density. In addition, four higher-rank tensor densities will appear. One of the possible definitions of the additional tensor densities is such that their contraction with a Kronecker symbol gives $Q_{s}(\mathbf{r}), \mathbf{S}_{q}(\mathbf{r})$, $\Pi_{q}(\mathbf{r})$ and $V_{q, \mu \nu}(\mathbf{r})$, but there are many other possible definitions that are all related through the recoupling of derivatives similar in spirit to Eq. (59). We feel it is important that we are able to make an informed choice of the local densities used to construct an energy density. In our view, an ideal choice would be to keep only irreducible and non-redundant densities, and preferably those that have some practical advantages such as requiring less memory storage compared to other possible choices.

As a third problem, the components of the two normal tensor densities $\tau_{q, \mu \nu}(\mathbf{r})$ and $K_{q, \mu \nu \kappa}(\mathbf{r})$ introduced in Sec. IIB 4 do not all behave in the same way under timereversal, such that one cannot attribute a definite global behavior under time-reversal to these densities. This can ultimately be traced back to the fact that these local densities are generated by a non-hermitian combination of gradient operators $\left(\nabla_{\mu} \nabla_{\nu}^{\prime}\right)$ and therefore are complexvalued. This makes separating the densities into timeeven and time-odd categories impossible, complicates the separation of terms of the EDF as either time-even or time-odd 4 and explains why this has not been done in Eq. (5).

For the pair densities, this issue already arises at leading order, but the literature has so far simply accepted this inconvenient feature, see for example Refs. [11, 19]. We would prefer a prescription that guarantees the reality of all densities, and therefore make

\footnotetext{
4 This issue was overlooked in the earlier Ref. 34], where a term bilinear in $\tau_{q, \mu \nu}(\mathbf{r})$ was wrongly assigned as time-even and a term bilinear in $K_{q, \mu \nu \kappa}(\mathbf{r})$ was wrongly assigned as time-odd.
} 
a clear delineation of time-even and time-odd possible for both types of densities and terms of the EDF.

In the next section, we will introduce a definition and a notation for normal and pair densities that is extensible to any order of derivatives and that guarantees real densities that have well-defined signs under timereversal. In addition, it will help us identify redundancies and relations between different densities by making their operator structure explicit.

\section{A NEW NOTATION AND DEFINITION FOR LOCAL DENSITIES}

\section{A. Systematic counting notation}

\section{Normal densities}

In order to approach the problem of defining local normal and pair densities in a more systematic way, we propose the following definition for normal densities obtained by applying derivatives to the non-local density $\rho\left(\mathbf{r}, \mathbf{r}^{\prime}\right)$ and then setting the positions $\mathbf{r}$ and $\mathbf{r}^{\prime}$ to be equal

$$
\begin{aligned}
& \left.D_{q}^{A, B}(\mathbf{r}) \equiv \operatorname{Re}\left\{\hat{A}^{\prime} \hat{B} \rho_{q}\left(\mathbf{r}, \mathbf{r}^{\prime}\right)\right\}\right|_{\mathbf{r}=\mathbf{r}^{\prime}} \\
& \left.C_{q}^{A, B}(\mathbf{r}) \equiv \operatorname{Im}\left\{\hat{A}^{\prime} \hat{B} \rho_{q}\left(\mathbf{r}, \mathbf{r}^{\prime}\right)\right\}\right|_{\mathbf{r}=\mathbf{r}^{\prime}}
\end{aligned}
$$

where the "left" operator $\hat{A}^{\prime}$ is a combination of derivatives acting on the primed spatial coordinates in the non-local density, whereas the "right" operator $\hat{B}$ is a combination of derivatives acting on the spatial coordinates of the unprimed coordinates.

Similarly, we also define $D$ and $C$ objects starting from the non-local spin density $\mathbf{s}_{q}\left(\mathbf{r}, \mathbf{r}^{\prime}\right)$

$$
\begin{aligned}
& \left.D_{q}^{A, B \sigma}(\mathbf{r}) \equiv \operatorname{Re}\left\{\hat{A}^{\prime} \hat{B} \mathbf{s}_{q}\left(\mathbf{r}, \mathbf{r}^{\prime}\right)\right\}\right|_{\mathbf{r}=\mathbf{r}^{\prime}}, \\
& \left.C_{q}^{A, B \sigma}(\mathbf{r}) \equiv \operatorname{Im}\left\{\hat{A}^{\prime} \hat{B} \mathbf{s}_{q}\left(\mathbf{r}, \mathbf{r}^{\prime}\right)\right\}\right|_{\mathbf{r}=\mathbf{r}^{\prime}} .
\end{aligned}
$$

The generic definitions made in Eqs. (60) -63) omit the tensor structure of the densities and currents on the lefthand side as they are specific to each choice of operators $\hat{A}$ and $\hat{B}$. The indices labeling the Cartesian components of the densities and currents can easily be accommodated in this kind of notation through subscripts: as an example we write

$$
D_{q, \mu \nu \kappa}^{\nabla, \nabla \sigma}(\mathbf{r})=\left.\operatorname{Re}\left\{\nabla_{\mu}^{\prime} \nabla_{\nu} s_{q, \kappa}\left(\mathbf{r}, \mathbf{r}^{\prime}\right)\right\}\right|_{\mathbf{r}=\mathbf{r}^{\prime}} .
$$

Note that the order of subscripts follows the order of operators in the superscripts. Tensor contractions with Kronecker symbols, i.e. scalar products between the operators $\hat{A}$ and $\hat{B}$ in the definition of the local densities, can be accommodated with the use of brackets; we define for example

$$
D_{q, \kappa}^{(\nabla, \nabla) \sigma}(\mathbf{r})=\sum_{\mu} D_{q, \mu \mu \kappa}^{\nabla, \nabla \sigma}(\mathbf{r})
$$

$$
\begin{aligned}
& =\left.\sum_{\mu} \operatorname{Re}\left\{\nabla_{\mu}^{\prime} \nabla_{\mu} s_{q, \kappa}\left(\mathbf{r}, \mathbf{r}^{\prime}\right)\right\}\right|_{\mathbf{r}=\mathbf{r}^{\prime}} \\
& =\left.\operatorname{Re}\left\{\nabla^{\prime} \cdot \nabla s_{q, \kappa}\left(\mathbf{r}, \mathbf{r}^{\prime}\right)\right\}\right|_{\mathbf{r}=\mathbf{r}^{\prime}} .
\end{aligned}
$$

Similarly, as a shorthand for vector products, we introduce

$$
\begin{aligned}
C_{q, \mu}^{1, \nabla \times \sigma}(\mathbf{r}) & =\left.\sum_{\nu \kappa} \operatorname{Im}\left\{\epsilon_{\mu \nu \kappa} \nabla_{\nu} s_{q, \kappa}\left(\mathbf{r}, \mathbf{r}^{\prime}\right)\right\}\right|_{\mathbf{r}=\mathbf{r}^{\prime}} \\
\Leftrightarrow \quad \mathbf{C}_{q}^{1, \nabla \times \sigma}(\mathbf{r}) & =\left.\operatorname{Im}\left\{\nabla \times \mathbf{s}_{q}\left(\mathbf{r}, \mathbf{r}^{\prime}\right)\right\}\right|_{\mathbf{r}=\mathbf{r}^{\prime}},
\end{aligned}
$$

where $\epsilon_{\mu \nu \kappa}$ is the skew-symmetric rank-3 Levi-Civita tensor.

The definitions (60)- (63) automatically lead to local densities that are a real function of the position $\mathbf{r}$ and that are either even or odd under time-reversal. In addition, in this form they can be efficiently implemented in numerical codes. For formal manipulations, such as the derivation of the single-particle Hamiltonian from the EDF that will be sketched in Sec. IVC, or the derivation of the residual interaction as used in QRPA from the EDF, or the construction of the MR extension of the EDF, it is necessary to use an alternative definition that does not necessitate to take the real or imaginary part of some expression.

Using that the real and imaginary parts of a complex number can be obtained as $\operatorname{Re}\{z\}=\frac{1}{2}\left(z+z^{*}\right)$ and $\operatorname{Im}\{z\}=-\frac{i}{2}\left(z-z^{*}\right)$, respectively, and exploiting the symmetry relation $\rho_{q}\left(\mathbf{r}, \mathbf{r}^{\prime}\right)=\rho_{q}^{*}\left(\mathbf{r}^{\prime}, \mathbf{r}\right)$ of the non-local density (15), the expressions (60) and (61) for the local densities $D_{q}^{A, B}(\mathbf{r})$ and $C_{q}^{A, B}(\mathbf{r})$ can be rewritten in terms of an hermitian or anti-hermitian combination of the operators $\hat{A}$ and $\hat{B}$

$$
\begin{aligned}
D_{q}^{A, B}(\mathbf{r}) & =\left.\operatorname{Re}\left\{\hat{A}^{\prime} \hat{B} \rho_{q}\left(\mathbf{r}, \mathbf{r}^{\prime}\right)\right\}\right|_{\mathbf{r}=\mathbf{r}^{\prime}} \\
& =\left.\hat{A}^{\prime} \hat{B} \frac{1}{2}\left[\rho_{q}\left(\mathbf{r}, \mathbf{r}^{\prime}\right)+\rho_{q}^{*}\left(\mathbf{r}, \mathbf{r}^{\prime}\right)\right]\right|_{\mathbf{r}=\mathbf{r}^{\prime}} \\
& =\left.\frac{1}{2}\left[\hat{A}^{\prime} \hat{B} \rho_{q}\left(\mathbf{r}, \mathbf{r}^{\prime}\right)+\hat{A} \hat{B}^{\prime} \rho_{q}^{*}\left(\mathbf{r}^{\prime}, \mathbf{r}\right)\right]\right|_{\mathbf{r}=\mathbf{r}^{\prime}} \\
& =\left.\frac{1}{2}\left(\hat{A}^{\prime} \hat{B}+\hat{A} \hat{B}^{\prime}\right) \rho_{q}\left(\mathbf{r}, \mathbf{r}^{\prime}\right)\right|_{\mathbf{r}=\mathbf{r}^{\prime}} \\
C_{q}^{A, B}(\mathbf{r}) & =\left.\operatorname{Im}\left\{\hat{A}^{\prime} \hat{B} \rho_{q}\left(\mathbf{r}, \mathbf{r}^{\prime}\right)\right\}\right|_{\mathbf{r}=\mathbf{r}^{\prime}} \\
& =\left.\hat{A}^{\prime} \hat{B}\left(-\frac{\mathrm{i}}{2}\right)\left[\rho_{q}\left(\mathbf{r}, \mathbf{r}^{\prime}\right)-\rho_{q}^{*}\left(\mathbf{r}, \mathbf{r}^{\prime}\right)\right]\right|_{\mathbf{r}=\mathbf{r}^{\prime}} \\
& =-\left.\frac{i}{2}\left[\hat{A}^{\prime} \hat{B} \rho_{q}\left(\mathbf{r}, \mathbf{r}^{\prime}\right)-\hat{A} \hat{B}^{\prime} \rho_{q}^{*}\left(\mathbf{r}^{\prime}, \mathbf{r}\right)\right]\right|_{\mathbf{r}=\mathbf{r}^{\prime}} \\
& =-\left.\frac{\mathrm{i}}{2}\left(\hat{A}^{\prime} \hat{B}-\hat{A} \hat{B}^{\prime}\right) \rho_{q}\left(\mathbf{r}, \mathbf{r}^{\prime}\right)\right|_{\mathbf{r}=\mathbf{r}^{\prime}}
\end{aligned}
$$

When going from the second to the third line in each of these manipulations, the coordinates $\mathbf{r}$ and $\mathbf{r}^{\prime}$ are exchanged in the second term, which can be done when both coordinates are to be set equal afterwards.

The non-local spin density exhibits the same symmetry $\mathbf{s}_{q}\left(\mathbf{r}, \mathbf{r}^{\prime}\right)=\mathbf{s}_{q}^{*}\left(\mathbf{r}^{\prime}, \mathbf{r}\right)$ under exchange of $\mathbf{r}$ and $\mathbf{r}^{\prime}$ as $\rho_{q}\left(\mathbf{r}, \mathbf{r}^{\prime}\right)$, Eq. (15), therefore the symmetrized expressions 
for $D_{q, \kappa}^{A, B \sigma}(\mathbf{r})$ and $C_{q, \kappa}^{A, B \sigma}(\mathbf{r})$ are obtained with the same manipulations and take the same structure as their homologues without spin

$$
\begin{aligned}
D_{q, \kappa}^{A, B \sigma}(\mathbf{r}) & =\left.\operatorname{Re}\left\{\hat{A}^{\prime} \hat{B} s_{q, \kappa}\left(\mathbf{r}, \mathbf{r}^{\prime}\right)\right\}\right|_{\mathbf{r}=\mathbf{r}^{\prime}} \\
& =\left.\frac{1}{2}\left(\hat{A}^{\prime} \hat{B}+\hat{A} \hat{B}^{\prime}\right) s_{q, \kappa}\left(\mathbf{r}, \mathbf{r}^{\prime}\right)\right|_{\mathbf{r}=\mathbf{r}^{\prime}} \\
C_{q, \kappa}^{A, B \sigma}(\mathbf{r}) & =\left.\operatorname{Im}\left\{\hat{A}^{\prime} \hat{B} s_{q, \kappa}\left(\mathbf{r}, \mathbf{r}^{\prime}\right)\right\}\right|_{\mathbf{r}=\mathbf{r}^{\prime}} \\
& =-\left.\frac{i}{2}\left(\hat{A}^{\prime} \hat{B}-\hat{A} \hat{B}^{\prime}\right) s_{q, \kappa}\left(\mathbf{r}, \mathbf{r}^{\prime}\right)\right|_{\mathbf{r}=\mathbf{r}^{\prime}}
\end{aligned}
$$

From the hermiticity of the density matrix $\rho_{j k}=\rho_{k j}^{*}$ and of the kernels $\Psi_{j}^{\dagger}\left(\mathbf{r}^{\prime}\right) \Psi_{k}(\mathbf{r})=\left[\Psi_{k}^{\dagger}(\mathbf{r}) \Psi_{j}\left(\mathbf{r}^{\prime}\right)\right]^{*}$ and $\Psi_{j}^{\dagger}\left(\mathbf{r}^{\prime}\right) \hat{\sigma}_{\mu} \Psi_{k}(\mathbf{r})=\left[\Psi_{k}^{\dagger}(\mathbf{r}) \hat{\sigma}_{\mu} \Psi_{j}\left(\mathbf{r}^{\prime}\right)\right]^{*}$, follows that the combined contributions of the states $j$ and $k$ to the local densities (60) - 63) through the expansion of the non-local densities into single-particle states, Eqs. (19) and (20), that is $\left\{\rho_{j k} \Psi_{j}^{\dagger}(\mathbf{r}) \Psi_{k}(\mathbf{r})+\rho_{k j} \Psi_{k}^{\dagger}(\mathbf{r}) \Psi_{j}(\mathbf{r})\right\}$ and $\left\{\rho_{j k} \Psi_{j}^{\dagger}(\mathbf{r}) \hat{\sigma}_{\mu} \Psi_{k}(\mathbf{r})+\rho_{k j} \Psi_{k}^{\dagger}(\mathbf{r}) \hat{\sigma}_{\mu} \Psi_{j}(\mathbf{r})\right\}$, are both real. This has been implicitly used when attributing the real and imaginary part of operators $\hat{A}$ and $\hat{B}$ acting on some part of these objects to the sum and difference of the operators as done in Eqs. (67)-(70). The possibility to do so is limited to normal densities, which will lead to a fundamental difference between normal densities and the pair densities that are defined in the next subsection. In the canonical single-particle basis, $\rho$ is diagonal [2] and all contributions to a local, normal density from any individual single-particle state are even automatically real. In fact, in this basis, the densities can be efficiently calculated as

$$
\begin{gathered}
D_{q}^{A, B}(\mathbf{r})=\sum_{k} \rho_{k k} \operatorname{Re}\left\{\left[\hat{A} \Psi_{k}^{\dagger}(\mathbf{r})\right]\left[\hat{B} \Psi_{k}(\mathbf{r})\right]\right\}, \\
C_{q}^{A, B}(\mathbf{r})=\sum_{k} \rho_{k k} \operatorname{Im}\left\{\left[\hat{A} \Psi_{k}^{\dagger}(\mathbf{r})\right]\left[\hat{B} \Psi_{k}(\mathbf{r})\right]\right\}, \\
D_{q, k}^{A, B \sigma}(\mathbf{r})=\sum_{k} \rho_{k k} \operatorname{Re}\left\{\left[\hat{A} \Psi_{k}^{\dagger}(\mathbf{r})\right] \hat{\sigma}_{\kappa}\left[\hat{B} \Psi_{k}(\mathbf{r})\right]\right\}, \\
C_{q, k}^{A, B \sigma}(\mathbf{r})=\sum_{k} \rho_{k k} \operatorname{Im}\left\{\left[\hat{A} \Psi_{k}^{\dagger}(\mathbf{r})\right] \hat{\sigma}_{\kappa}\left[\hat{B} \Psi_{k}(\mathbf{r})\right]\right\},
\end{gathered}
$$

where the sum runs over the canonical single-particle states $k$ of the nucleon species $q$. The adaptation of these expressions to arbitrary single-particle bases is straightforward, but less efficient for numerical implementations because of the double summation over single-particle states that is avoided in the canonical basis.

\section{Pair densities}

The definition of local pair and spin-pair densities along the same lines requires a few additional considerations. These are necessary because of conceptual differences between normal and pair densities. Most importantly, all ingredients of the non-local pair densities $\tilde{\rho}_{q}\left(\mathbf{r}, \mathbf{r}^{\prime}\right)$ and $\tilde{\mathbf{s}}_{q}\left(\mathbf{r}, \mathbf{r}^{\prime}\right)$ as defined in Eqs. (21) and (22), that is the anomalous density matrix $\kappa_{k j}$ and the two-body wave functions $\tilde{\varrho}_{j k}\left(\mathbf{r}, \mathbf{r}^{\prime}\right)$ and $\tilde{\varsigma}_{j k, \mu}\left(\mathbf{r}, \mathbf{r}^{\prime}\right)$, transform as rank-two tensors under basis changes, and not as hermitian matrices. Related to this is that the two non-local pair densities $\tilde{\rho}_{q}\left(\mathbf{r}, \mathbf{r}^{\prime}\right)$ and $\tilde{\mathbf{s}}_{q}\left(\mathbf{r}, \mathbf{r}^{\prime}\right)$ not only transform differently from the normal non-local densities under time-reversal and exchange of $\mathbf{r} \leftrightarrow \mathbf{r}^{\prime}$, but also differently from each other, see Sec. IIB. There are also systematic differences between the behavior of normal and pair densities under other similarity transformations. In addition, the anomalous density matrix $\kappa_{k j}$ cannot always be chosen to be real, even in the canonical basis. All of this has consequences for the efficient definition of pair densities. More detailed explanations of these properties can be found in Appendix E. Taking them into account, we propose to define local pair densities as follows

$$
\begin{gathered}
\left.\tilde{D}_{q}^{A, B}(\mathbf{r}) \equiv \sum_{j<k} \kappa_{k j}\left(\hat{A}^{\prime} \hat{B}+\hat{A} \hat{B}^{\prime}\right) \operatorname{Re}\left\{\tilde{\varrho}_{j k}\left(\mathbf{r}, \mathbf{r}^{\prime}\right)\right\}\right|_{\mathbf{r}=\mathbf{r}^{\prime}}, \\
\left.\tilde{C}_{q}^{A, B}(\mathbf{r}) \equiv \sum_{j<k} \kappa_{k j}\left(\hat{A}^{\prime} \hat{B}+\hat{A} \hat{B}^{\prime}\right) \operatorname{Im}\left\{\tilde{\varrho}_{j k}\left(\mathbf{r}, \mathbf{r}^{\prime}\right)\right\}\right|_{\mathbf{r}=\mathbf{r}^{\prime}} \\
\left.\tilde{D}_{q, \mu}^{A, B \sigma}(\mathbf{r}) \equiv \sum_{j<k} \kappa_{k j}\left(\hat{A}^{\prime} \hat{B}-\hat{A} \hat{B}^{\prime}\right) \operatorname{Re}\left\{\tilde{\varsigma}_{j k, \mu}\left(\mathbf{r}, \mathbf{r}^{\prime}\right)\right\}\right|_{\mathbf{r}=\mathbf{r}^{\prime}}, \\
\left.\tilde{C}_{q, \mu}^{A, B \sigma}(\mathbf{r}) \equiv \sum_{j<k} \kappa_{k j}\left(\hat{A}^{\prime} \hat{B}-\hat{A} \hat{B}^{\prime}\right) \operatorname{Im}\left\{\tilde{\varsigma}_{j k, \mu}\left(\mathbf{r}, \mathbf{r}^{\prime}\right)\right\}\right|_{\mathbf{r}=\mathbf{r}^{\prime}}
\end{gathered}
$$

As done above for normal densities, the possible Cartesian tensor components of the operators $\hat{A}$ and $\hat{B}$ are suppressed for sake of a compact generic notation. Features of these definitions are

- Unlike the local normal densities defined through Eqs. (60)- 63), the local pair densities cannot be considered a priori to be real under all circumstances. They are, however, defined such that only the elements of $\kappa_{k j}$ are complex, not the spatial functions they multiply.

- For many situations of practical interest, such as static calculations that conserve some antilinear anti-hermitian symmetry [41], there exists a specific gauge in which the anomalous density matrix $\kappa_{i j}$ can be chosen to be real in the centerof-mass frame. This is the choice that is made in the majority of numerical codes for static selfconsistent mean-field calculations. In such case, all local pair densities defined through Eqs. (75)-(78) are real and have well-defined behavior under timereversal, see Eqs. (83)-(86) in what follows. 
- In one way or another, all conserved spatial symmetries of the single-particle states in the canonical basis of an HFB calculation are transferred to the local pair densities as defined through Eqs. (75)-(78), such that the local pair densities respect the same set of spatial symmetries as the local normal densities in a symmetryrestricted HFB code, see Appendix D.

- Contrarily to the normal densities, the distinction between $\tilde{D}$ and $\tilde{C}$ objects has to be made through the real and imaginary parts of $\tilde{\varrho}_{j k}\left(\mathbf{r}, \mathbf{r}^{\prime}\right)$ and $\tilde{\varsigma}_{j k, \mu}\left(\mathbf{r}, \mathbf{r}^{\prime}\right)$, instead of the sum or difference of the gradient operators. Because of the symmetries of $\kappa_{j k}, \tilde{\varrho}_{j k}\left(\mathbf{r}, \mathbf{r}^{\prime}\right)$ and $\tilde{\varsigma}_{j k, \mu}\left(\mathbf{r}, \mathbf{r}^{\prime}\right)$ under exchange of single-particle labels and positions, summing up a pair density with $\left(\hat{A}^{\prime} \hat{B}-\hat{A} \hat{B}^{\prime}\right)$ acting on $\tilde{\varrho}_{j k}\left(\mathbf{r}, \mathbf{r}^{\prime}\right)$ gives zero when setting $\mathbf{r}=\mathbf{r}^{\prime}$, as does doing the same with $\left(\hat{A}^{\prime} \hat{B}+\hat{A} \hat{B}^{\prime}\right)$ acting on $\tilde{\varsigma}_{j k, \mu}\left(\mathbf{r}, \mathbf{r}^{\prime}\right)$.

- The skew-symmetry of $\kappa_{j k},\left(\hat{A}^{\prime} \hat{B}+\hat{A} \hat{B}^{\prime}\right) \tilde{\varrho}_{j k}\left(\mathbf{r}, \mathbf{r}^{\prime}\right)$, and $\left(\hat{A}^{\prime} \hat{B}-\hat{A} \hat{B}^{\prime}\right) \tilde{\varsigma}_{j k, \mu}\left(\mathbf{r}, \mathbf{r}^{\prime}\right)$ under exchange of $j$ and $k$ has been used to reduce the summations in Eqs. (75)-78 to just half of the possible combinations of single-particle levels $j$ and $k$, which cancels an implicit factor factor $1 / 2$ from the symmetrization or anti-symmetrization of the operators $\hat{A}$ and $\hat{B}$.

- Unless $\hat{A}=\hat{B}$, the sums and differences of these operators in Eqs. (75)-(78) cannot be further simplified. With that, the definitions of the pair densities are much closer to Eqs. (67) - (70) than to the more efficient Eqs. (60) - 63) for normal densities.

\section{B. Useful relations}

In static calculations, all local normal densities $D$ and $C$ are real by construction, which implies that they all have definite behavior under time-reversal. One can easily show that, for arbitrary combinations of derivative operators $\hat{A}$ and $\hat{B}$,

$$
\begin{gathered}
{\left[D_{q}^{A, B}\right]^{T}(\mathbf{r})=+D_{q}^{A, B}(\mathbf{r}),} \\
{\left[C_{q}^{A, B}\right]^{T}(\mathbf{r})=-C_{q}^{A, B}(\mathbf{r}),} \\
{\left[D_{q}^{A, B \sigma}\right]^{T}(\mathbf{r})=-D_{q}^{A, B \sigma}(\mathbf{r}),} \\
{\left[C_{q}^{A, B \sigma}\right]^{T}(\mathbf{r})=+C_{q}^{A, B \sigma}(\mathbf{r}) .}
\end{gathered}
$$

Provided the anomalous density matrix $\kappa_{i j}$ can be chosen to be real, analogue equations also hold for the local pair densities

$$
\begin{aligned}
& {\left[\tilde{D}_{q}^{A, B}\right]^{T}(\mathbf{r})=+\tilde{D}_{q}^{A, B}(\mathbf{r}),} \\
& {\left[\tilde{C}_{q}^{A, B}\right]^{T}(\mathbf{r})=-\tilde{C}_{q}^{A, B}(\mathbf{r}),}
\end{aligned}
$$

$$
\begin{aligned}
& {\left[\tilde{D}_{q}^{A, B \sigma}\right]^{T}(\mathbf{r})=-\tilde{D}_{q}^{A, B \sigma}(\mathbf{r}),} \\
& {\left[\tilde{C}_{q}^{A, B \sigma}\right]^{T}(\mathbf{r})=+\tilde{C}_{q}^{A, B \sigma}(\mathbf{r}) .}
\end{aligned}
$$

The local densities defined here have a few more practical properties. The first is that they exhibit definite signs under the exchange of left and right operators

$$
\begin{aligned}
D_{q}^{A, B}(\mathbf{r}) & =+D_{q}^{B, A}(\mathbf{r}), & C_{q}^{A, B}(\mathbf{r}) & =-C_{q}^{B, A}(\mathbf{r}), \\
D_{q}^{A, B \sigma}(\mathbf{r}) & =+D_{q}^{B, A \sigma}(\mathbf{r}), & C_{q}^{A, B \sigma}(\mathbf{r}) & =-C_{q}^{B, A \sigma}(\mathbf{r}), \\
\tilde{D}_{q}^{A, B}(\mathbf{r}) & =+\tilde{D}_{q}^{B, A}(\mathbf{r}), & \tilde{C}_{q}^{A, B}(\mathbf{r}) & =+\tilde{C}_{q}^{B, A}(\mathbf{r}), \\
\tilde{D}_{q}^{A, B \sigma}(\mathbf{r}) & =-\tilde{D}_{q}^{B, A \sigma}(\mathbf{r}), & \tilde{C}_{q}^{A, B \sigma}(\mathbf{r}) & =-\tilde{C}_{q}^{B, A \sigma}(\mathbf{r}),
\end{aligned}
$$

where it is implied that the tensor indices in the subscripts need to be exchanged accordingly. These relations are consequences of the symmetries of the nonlocal densities under exchange of the positions $\mathbf{r}$ and $\mathbf{r}^{\prime}$, Eqs. (15) and (16). They imply that some densities and currents are automatically zero when the generating operators $\hat{A}$ and $\hat{B}$ are equal, examples being $C_{q}^{A, A}(\mathbf{r})=$ $C_{q}^{A, A \sigma}(\mathbf{r})=0$ and $\tilde{D}_{q}^{A, A \sigma}(\mathbf{r})=\tilde{C}_{q}^{A, A \sigma}(\mathbf{r})=0$. The latter, however is only found when not mixing protons and neutrons [44, 51].

Another set of relations that will be useful for the identification of reducible densities in Sec. [IID 1 are the following equalities,

$$
\begin{aligned}
& D_{q}^{\nabla A, B}(\mathbf{r})=\nabla D_{q}^{A, B}(\mathbf{r})-D_{q}^{A, \nabla B}(\mathbf{r}), \\
& C_{q}^{\nabla A, B}(\mathbf{r})=\nabla C_{q}^{A, B}(\mathbf{r})-C_{q}^{A, \nabla B}(\mathbf{r}) .
\end{aligned}
$$

Equations (88) and (89) follow directly from the chain rule for derivatives and analogous relations hold for the spin, pair and spin pair densities and currents.

\section{Dictionary}

Having explored the consequences of this new notation and the sometimes new definitions that it implies, we are now in a position to rewrite the densities employed in Ref. [36 using the new notation. We have chosen the letters "D" and "C" for these objects as shorthands for density and current respectively. For normal densities, the $C$ objects, Eqs. 610 and 663) do indeed resemble currents. For example, $C_{\mu}^{1, \nabla}(\mathbf{r})$ can be identified with

$$
\begin{aligned}
C_{\mu}^{1, \nabla}(\mathbf{r}) & =\left.\operatorname{Im}\left\{\nabla_{\mu} \rho_{q}\left(\mathbf{r}, \mathbf{r}^{\prime}\right)\right\}\right|_{\mathbf{r}=\mathbf{r}^{\prime}} \\
& =-\left.\frac{i}{2}\left[\nabla_{\mu} \rho_{q}\left(\mathbf{r}, \mathbf{r}^{\prime}\right)-\nabla_{\mu} \rho_{q}^{*}\left(\mathbf{r}, \mathbf{r}^{\prime}\right)\right]\right|_{\mathbf{r}=\mathbf{r}^{\prime}} \\
& =-\left.\frac{i}{2}\left(\nabla_{\mu}-\nabla_{\mu}^{\prime}\right) \rho_{q}\left(\mathbf{r}^{\prime}, \mathbf{r}\right)\right|_{\mathbf{r}=\mathbf{r}^{\prime}} \\
& =j_{\mu}(\mathbf{r}) .
\end{aligned}
$$

Similarly, all other local densities used in Eq. (5) and elsewhere in the literature can be unambiguously 
expressed in the new notation. At leading and next-toleading order, every normal density can be identified with exactly one $D$ or $C$ object, matching their generating operator structure

$$
\begin{array}{rlrl}
\rho_{q}(\mathbf{r}) & \rightarrow D_{q}^{1,1}(\mathbf{r}), & & s_{q, \mu}(\mathbf{r}) \rightarrow D_{q, \mu}^{1, \sigma}(\mathbf{r}), \\
\tau_{q}(\mathbf{r}) & \rightarrow D_{q}^{(\nabla, \nabla)}(\mathbf{r}), & T_{q, \mu}(\mathbf{r}) & \rightarrow D_{q, \mu}^{(\nabla, \nabla) \sigma}(\mathbf{r}), \\
& F_{q, \mu}(\mathbf{r}) \rightarrow D_{q, \mu}^{\nabla,(\nabla \sigma)}(\mathbf{r}), \\
j_{q, \mu}(\mathbf{r}) & \rightarrow C_{q, \mu}^{1, \nabla}(\mathbf{r}), & J_{q, \mu \nu}(\mathbf{r}) & \rightarrow C_{q, \mu \nu}^{1, \nabla \sigma}(\mathbf{r}) .
\end{array}
$$

As $\tilde{\rho}_{q}(\mathbf{r}), \tilde{J}_{q, \mu \nu}(\mathbf{r})$, and $\tilde{\tau}_{q}(\mathbf{r})$ are all split in two separate objects, there is no one-to-one correspondence between the traditional and the new notation. Instead, one finds

$$
\begin{aligned}
\tilde{\rho}_{q}(\mathbf{r}) & \rightarrow \tilde{D}_{q}^{1,1}(\mathbf{r})+\mathrm{i} \tilde{C}_{q}^{1,1}(\mathbf{r}), \\
\tilde{\tau}_{q}(\mathbf{r}) & \rightarrow \tilde{D}_{q}^{(\nabla, \nabla)}(\mathbf{r})+\mathrm{i} \tilde{C}_{q}^{(\nabla, \nabla)}(\mathbf{r}), \\
\tilde{J}_{q, \mu \nu}(\mathbf{r}) & \rightarrow \tilde{C}_{q, \mu \nu}^{1, \nabla \sigma}(\mathbf{r})-\mathrm{i} \tilde{D}_{q, \mu \nu}^{1, \nabla \sigma}(\mathbf{r}) .
\end{aligned}
$$

The additional sign that accompanies $\tilde{D}_{q, \mu \nu}^{1, \nabla \sigma}(\mathbf{r})$, follows from the symmetry (26) of the $S=1$ two-body wave function $\tilde{\varsigma}_{j k, \mu}\left(\mathbf{r}, \mathbf{r}^{\prime}\right)$. The separation of the traditional pair densities into a $\tilde{D}$ and $\tilde{C}$ object separates the real and imaginary parts of the operators acting on the two-body wave functions $\tilde{\varrho}_{j k}\left(\mathbf{r}, \mathbf{r}^{\prime}\right)$ and $\tilde{\varsigma}_{j k, \mu}\left(\mathbf{r}, \mathbf{r}^{\prime}\right)$ in Eqs. (75)-(78). But the $\tilde{D}$ and $\tilde{C}$ objects still can be complex when $\kappa_{j k}$ entering the same equations is complex.

For four of the normal densities that appear in the N2LO functional of [36], Eq. (5), that is $Q_{q}(\mathbf{r}), V_{q, \mu \nu}(\mathbf{r})$, $\mathbf{S}_{q}(\mathbf{r})$ and $\boldsymbol{\Pi}_{q}(\mathbf{r})$, the mapping is one-to-one as well:

$$
\begin{array}{rlrl}
Q_{q}(\mathbf{r}) & \rightarrow D_{q}^{\Delta, \Delta}(\mathbf{r}), & S_{q, \mu}(\mathbf{r}) & \rightarrow D_{q, \mu}^{\Delta, \Delta \sigma}(\mathbf{r}), \\
\Pi_{q, \mu}(\mathbf{r}) & \rightarrow C_{q, \mu}^{(\nabla, \nabla) \nabla}(\mathbf{r}), \quad V_{q, \mu \nu}(\mathbf{r}) & \rightarrow C_{q, \mu \nu}^{(\nabla, \nabla) \nabla \sigma}(\mathbf{r}) .
\end{array}
$$

The densities $\tau_{q, \mu \nu}(\mathbf{r})$ and $K_{q, \mu \nu \kappa}(\mathbf{r})$ that also appear in the N2LO functional of Ref. [36], Eq. (5), can only be written as a sum of a $D$ and a $C$ object each, reflecting the fact that they are neither time-even nor time-odd. We have for these

$$
\begin{aligned}
\tau_{q, \mu \nu}(\mathbf{r}) & \rightarrow D_{q, \nu \mu}^{\nabla, \nabla}(\mathbf{r})+\mathrm{i} C_{q, \nu \mu}^{\nabla, \nabla}(\mathbf{r}), \\
K_{q, \mu \nu \kappa}(\mathbf{r}) & \rightarrow D_{q, \nu \mu \kappa}^{\nabla, \nabla \sigma}(\mathbf{r})+\mathrm{i} C_{q, \nu \mu \kappa}^{\nabla, \nabla \sigma}(\mathbf{r}),
\end{aligned}
$$

where Eq. (89) can be used to further reduce the currents

$$
\begin{aligned}
C_{q, \nu \mu}^{\nabla, \nabla}(\mathbf{r}) & =\frac{1}{2}\left[\nabla_{\nu} C_{q, \mu}^{1, \nabla}(\mathbf{r})-\nabla_{\mu} C_{q, \nu}^{1, \nabla}(\mathbf{r})\right], \\
C_{q, \nu \mu \kappa}^{\nabla, \nabla \sigma}(\mathbf{r}) & =\frac{1}{2}\left[\nabla_{\nu} C_{q, \mu}^{1, \nabla \sigma}(\mathbf{r})-\nabla_{\mu} C_{q, \nu}^{1, \nabla \sigma}(\mathbf{r})\right] .
\end{aligned}
$$

Eq. (96) is nothing but Eq. (57) in the new notation. This type of formal transformation will be discussed from a more general point of view in Sec. IIID1 Note that the indices $\mu \nu$ in Eqs. (94) and (95) are inverted on the right hand side as compared to the left hand side: the ordering of the indices in the traditional notation for $\tau_{q, \mu \nu}(\mathbf{r})$ and
$K_{q, \mu \nu \kappa}(\mathbf{r})$ as defined through Eqs. (46) and (47) is based on the ordering of the spatial and spin arguments of the non-local density matrices on the left-hand side of Eqs. (7) and (8), such that one starts with the indices of the components of the operators acting on non-primed coordinates followed by the indices of the components of the operators acting on primed coordinates. Our notation is based on the ordering of the spatial and spin arguments of creation and annihilation operators in the matrix elements on the right-hand side of Eqs. (7) and (8), such that it starts with the indices labeling the Cartesian tensor components of the operator $\hat{A}$ followed by the indices labeling the tensor components of the operator $\hat{B}$.

The N2LO pair densities are complex in general as well, and also need to be replaced by a combination of $\tilde{D}$ and $\tilde{C}$ objects:

$$
\begin{aligned}
\tilde{\tau}_{q, \mu \nu}(\mathbf{r}) & \rightarrow \tilde{D}_{q, \nu \mu}^{\nabla, \nabla}(\mathbf{r})+\mathrm{i} \tilde{C}_{q, \nu \mu}^{\nabla, \nabla}(\mathbf{r}), \\
\tilde{V}_{q, \mu \nu}(\mathbf{r}) & \rightarrow \tilde{C}_{q, \mu \nu}^{(\nabla, \nabla) \nabla \sigma}(\mathbf{r})-\mathrm{i} \tilde{D}_{q, \mu \nu}^{(\nabla, \nabla) \nabla \sigma}(\mathbf{r}), \\
\tilde{Q}_{q}(\mathbf{r}) & \rightarrow \tilde{D}_{q}^{\Delta, \Delta}(\mathbf{r})+\mathrm{i} \tilde{C}_{q}^{\Delta, \Delta}(\mathbf{r}),
\end{aligned}
$$

where we emphasize again the reordering of indices for $\tilde{\tau}_{q, \mu \nu}(\mathbf{r})$ as for $\tau_{q, \mu \nu}(\mathbf{r})$. The various $\tilde{D}$ or $\tilde{C}$ objects used to express pair densities have the same operator structure as the homologous normal densities, but they do not have the same interpretation in terms of what is usually called density and current. The reason is that the definition of pair densities sums up the local parts of two-body wave functions that provide an efficient factorization of specific traces of the two-body density matrix of a paired quasiparticle vacuum and that enter the pairing energy as obtained from contact interactions [49]. While the local normal densities are truly one-body objects that do not only factorize the particle-hole part of the N2LO EDF (and some other many-body operators), but also can be used to directly calculate numerous one-body observables, the pair densities only appear in matrix elements that are of two-body (or higher) nature.

As a conclusion to this section we see four main advantages of the $D(\mathbf{r}), C(\mathbf{r})$ notation for normal and pair densities as compared to the extension of the historical notations to higher-order in gradients. These advantages are:

1. Extensibility: the notation can be extended indefinitely, up to any order in derivatives.

2. Clarity: the operator structure of any given density is incorporated directly into the notation.

3. Reality: By construction, all normal $D(\mathbf{r})$ and $C(\mathbf{r})$ objects are real spatial functions when doing single-reference EDF calculations. Similarly, all pair densities $\tilde{D}(\mathbf{r})$ and $\tilde{C}(\mathbf{r})$ are real whenever $\kappa_{i j}$ can be chosen to be real, which is the case for the vast majority of use cases of interest for static mean-field calculations. 
4. Time-reversal: $D(\mathbf{r})$ and $C(\mathbf{r})$ objects have definite behavior under time-reversal: they are either time-even or time-odd, never mixed.

Taken together, these aspects make the $D(\mathbf{r})$ and $C(\mathbf{r})$ notation significantly easier to work with for functionals at N2LO and higher order. The new notation eliminates the need for ever-expanding lists of letters for densities, whose precise definition and symmetry properties can easily be confused both in writing and in practical implementations. The same advantages also carry over to the definition and notation of local potentials entering the single-particle Hamiltonian and the pair potentials, which will be addressed in Sec. IVC and Sec. IVE. respectively.

\section{Advantageous choices of densities}

\section{Redundancy and reducibility}

The recoupling relations, Eqs. (88) and (89) have striking implications on the redundancy and reducibility of the local densities. To start, we consider a spinless normal density $D$, with $a+b=N, N>0$, total gradient operators, which are split into $a$ gradients acting on the primed coordinate and $b$ gradients acting on the unprimed coordinate. If $a$ is non-zero, through repeated application of Eq. (88) we get

$$
D_{q, \mu_{1} \mu_{2} \ldots \mu_{a+b}}^{\nabla^{a}, \nabla^{b}}(\mathbf{r})=(-1)^{a} D_{q, \mu_{1} \mu_{2} \ldots \mu_{a+b}}^{1, \nabla^{a+b}}(\mathbf{r})+\sum_{n=1}^{a}(-1)^{n+1}\left(\prod_{k=1}^{n} \nabla_{\mu_{k}}\right) D_{q, \mu_{n+1} \ldots \mu_{a+b}}^{\nabla^{a-n}, \nabla^{b}}(\mathbf{r}) .
$$

If $b$ is non-zero, we have that

$$
D_{q, \mu_{1} \mu_{2} \ldots \mu_{a+b}}^{\nabla^{a}, \nabla^{b}}(\mathbf{r})=(-1)^{b} D_{q, \mu_{1} \mu_{2} \ldots \mu_{a+b}}^{\nabla_{a+b}, 1}(\mathbf{r})+\sum_{n=1}^{b}(-1)^{n+1}\left(\prod_{k=0}^{n-1} \nabla_{\mu_{a+b-k}}\right) D_{q, \mu_{1} \mu_{2} \ldots \mu_{a+b-n}}^{\nabla^{a}, \nabla^{b-n}}(\mathbf{r}) .
$$

If both $a$ and $b$ are non-zero, we can sum Eqs. (99) and (100), and use Eq. (15) to obtain

$$
\begin{aligned}
& 2 D_{q, \mu_{1} \mu_{2} \ldots \mu_{a+b}}^{\nabla^{a}, \nabla^{b}}(\mathbf{r}) \\
& \quad=\left[(-1)^{a}+(-1)^{b}\right] D_{q, \mu_{1} \mu_{2} \ldots \mu_{a+b}}^{1, \nabla^{a+b}}(\mathbf{r})+\cdots,
\end{aligned}
$$

where we have dropped terms involving derivatives of lower order densities. When $a+b$ is an odd number, the first term on the rhs in this equation vanishes, meaning that $D^{\nabla^{a}, \nabla^{b}}(\mathbf{r})$ can be rewritten in terms of gradients of lower-order densities. Hence, if the total number of derivatives in $D^{A, B}(\mathbf{r})$ is odd, the density is reducible. This result is valid as well when either $a$ or $b$ (but not both) are zero: in that case either Eq. (99) or Eq. (100) implies this result by itself.

Arguments along the same line hold for currents, spin densities and spin currents, as well as all types of pair densities and currents, provided (i) one uses the correct symmetry relations for the exchange of $\mathbf{r}$ and $\mathbf{r}^{\prime}$ for the underlying non-local densities, Eqs. (15) and (16), and (ii) one does not use any index corresponding to a Pauli $\sigma$ matrix in the recoupling. The following statements summarize the reducibility of densities in terms of the number of derivatives in the operator structure:

$$
\text { If } a+b \text { is odd, then }\left\{\begin{array}{l}
D_{q}^{\nabla^{a}, \nabla^{b}}(\mathbf{r}) \\
D_{q}^{\nabla^{a}, \nabla^{b} \sigma}(\mathbf{r}) \\
\tilde{D}_{q}^{\nabla^{a}, \nabla^{b}}(\mathbf{r}) \\
\tilde{C}_{q}^{\nabla^{a}, \nabla^{b}}(\mathbf{r})
\end{array}\right\} \text { is reducible. }
$$

$$
\text { If } a+b \text { is even, then }\left\{\begin{array}{l}
C_{q}^{\nabla^{a}, \nabla^{b}}(\mathbf{r}) \\
C_{q}^{\nabla^{a}, \nabla^{b} \sigma}(\mathbf{r}) \\
\tilde{D}_{q}^{\nabla^{a}, \nabla^{b} \sigma}(\mathbf{r}) \\
\tilde{C}_{q}^{\nabla^{a}, \nabla^{b} \sigma}(\mathbf{r})
\end{array}\right\} \text { is reducible. }
$$

It is thus possible to see immediately from the notation whether a density is reducible, and whether it can be eliminated from the definition of the functional. Eq. (57), which we used as an example of reducibility, is a particular case of these more general considerations.

Eq. (101) also implies a strong result with respect to the redundancy of any set of local densities: one out of any pair of densities $D^{\nabla^{a}, \nabla^{b}}, D^{\nabla^{a^{\prime}}, \nabla^{b^{\prime}}}$ of equal tensor rank is redundant, if the total number of gradients is equal, $a+b=a^{\prime}+b^{\prime}$. In other words, we need only pick at most a single spinless $D$ object at any given number of derivatives to allow for the most general EDF. As the analogue of Eq. (101) holds for the $C$ objects as well, we need at most one spinless $C$ object at that order; all others will automatically be redundant. The same reasoning holds for densities and currents with Pauli matrices. From this argument follows that going from $\mathrm{N}(\ell-1) \mathrm{LO}$ to N$\ell \mathrm{LO}$ in the Skyrme EDF one has to introduce at most four completely new normal densities: a scalar $D$ and a pseudoscalar $D_{\mu}^{\sigma}$ with $2 \ell$ gradients, as well as a vector $C_{\mu}$ and a pseudotensor $C_{\mu \nu}^{\sigma}$ with $2 \ell-1$ gradients. Similarly, to extend an N $(\ell-1)$ LO EDF to N $\ell$ LO, one needs at most four different pair densities: a $\tilde{D}$ and a $\tilde{C}$ with $2 \ell$ gradients, together with a $\tilde{D}_{\mu}^{\sigma}$ and a 
$\tilde{C}_{\mu}^{\sigma}$ with $2 \ell-1$ gradients, provided protons and neutrons are not mixed.

Combining the reducibility and the redundancy argument, we can simply enumerate the local densities we need, as we will do below.

\section{Computational considerations}

A final consideration in the selection of densities should be their efficiency of computation and storage. At LO and NLO, there is no particular reason for thinking about computational efficiency: the largest objects in terms of storage space are $J_{q, \mu \nu}(\mathbf{r})$ and its pair analogue $\tilde{J}_{q, \mu \nu}(\mathbf{r})$ which respectively represent 9 real and 9 complex (18 real) spatial functions. At N2LO however, storing the density $K_{q, \mu \nu \kappa}(\mathbf{r})$ completely requires 27 complex functions (54 real spatial functions), which can be reduced to 18 complex functions (36 real functions) when exploiting its symmetries with respect to permutations of the indices. The storage required for the local densities inevitably grows with increasing order in derivatives; while not necessarily being prohibitive at N2LO level yet, it is of practical interest to limit this growth where possible.

More problematic than the storage of the densities themselves is the storage of the derivatives of the singleparticle wave functions. For the NLO Skyrme functional, one needs only to calculate and store the single-particle wave functions themselves, their gradient and finally their Laplacian, but not the full set of their second derivatives. For the N2LO functional as formulated in Ref. [36] however, one needs to calculate and store all six linearly independent combinations of second derivatives of the single-particle wave functions when summing up the densities. For example, when written out in full as a sum over single-particle wave functions, the density $V_{q, \mu \nu}(\mathbf{r})$ as defined in Ref. [36] and used in Eq. (5) is given by

$$
V_{q, \mu \nu}(\mathbf{r})=\operatorname{Im}\left\{\sum_{j k} \rho_{k j} \sum_{\kappa}\left[\nabla_{\kappa} \Psi_{j}^{\dagger}(\mathbf{r})\right]\left[\nabla_{\kappa} \nabla_{\mu} \hat{\sigma}_{\nu} \Psi_{k}(\mathbf{r})\right]\right\} .
$$

Implementing the calculation of $V_{q, \mu \nu}(\mathbf{r})=C_{q, \mu \nu}^{(\nabla, \nabla) \nabla \sigma}(\mathbf{r})$ through Eq. (102) requires the calculation and storage of the second-order derivatives of the single-particle wave functions. They are required as well for the calculation of the current $\boldsymbol{\Pi}_{q}(\mathbf{r})=\mathbf{C}_{q}^{(\nabla, \nabla) \nabla}(\mathbf{r})$, and (if included in the pair functional) for the pair density $\tilde{V}_{q, \mu \nu}(\mathbf{r})$. This is a substantial amount of storage, as the single-particle wave functions are two-component complex-valued spinors and there are six independent second order derivatives to be calculated. As will be discussed in Sec. IVC, this matter can become even more critical when evaluating the action of the single-particle Hamiltonian on the single-particle states, as the terms originating from the variation of the EDF with respect to the density $V_{q, \mu \nu}(\mathbf{r})$ even require many combinations of third derivatives. This computational issue is particularly troubling for approaches that deal with large single-particle model spaces. In coordinate space approaches such as ours, calculating the derivatives on the mesh is the most timeconsuming task [45] and the storage of the single-particle wave functions and their derivatives dwarfs all other memory requirements.

For the N2LO functional, however, one can deduce that storage of all second-order derivatives is not necessary when making a suitable choice of densities. Indeed, using the recoupling relations we can rewrite Eq. (102) as

$$
\begin{aligned}
C_{q, \mu \nu}^{(\nabla, \nabla) \nabla \sigma}(\mathbf{r})= & -C_{q, \mu \nu}^{\Delta, \nabla \sigma}(\mathbf{r})+\frac{1}{2}\left[\Delta C_{q, \mu \nu}^{1, \nabla \sigma}(\mathbf{r})\right] \\
& -\frac{1}{2} \sum_{\kappa}\left[\nabla_{\kappa} \nabla_{\mu} C_{q, \kappa \nu}^{1, \nabla \sigma}(\mathbf{r})\right] .
\end{aligned}
$$

A direct implementation of Eq. (103) only requires the gradient and Laplacian of the single-particle wave functions for the calculation of the first term on the right hand side, while the two other terms are only external derivatives of densities of lower order that are already present in the NLO functional. Similar relations that avoid the storage of all second-order derivatives (other than the Laplacian) of the single-particle wave functions can easily be derived for $\boldsymbol{\Pi}_{q}(\mathbf{r})=\mathbf{C}_{q}^{(\nabla, \nabla) \nabla}(\mathbf{r})$ and $\tilde{V}_{q, \mu \nu}(\mathbf{r})$. An implementation that uses the original choice of densities of Ref. [36] requires the storage of ten sets of spinors (the wave functions, three first-order derivatives and six independent second-order derivatives) while an implementation that avoids $V_{q}(\mathbf{r}), \boldsymbol{\Pi}_{q}$ and $\tilde{V}_{q}(\mathbf{r})$ can make do with only half the memory: it requires only five sets of spinors. There is of course a corresponding gain in computational time: the first implementation requires the execution of nine derivative operations (three first-order ones and six second-order ones) while the second requires only six. If derivatives dominate the computational cost, as they do in our case, the CPU time required by the first implementation is roughly $50 \%$ larger than that required for the second implementation. Our experience is that this naive estimate is qualitatively correct for typical mesh sizes employed for heavy nuclei. While the differences in requirements might seem modest, we emphasize that the additional computational burden can be completely eliminated at no cost, i.e. both implementations perform an equivalent calculation.

The more general lesson to be learned here is that contractions of derivatives should be recoupled to Laplacians wherever possible. Further balancing the number of Laplacian operators on the "left" (acting on $\mathbf{r}^{\prime}$ ) and on the "right" (acting on $\mathbf{r}$ ) further helps reduce storage and computing costs. For example, the density $D_{q}^{1, \Delta \Delta}(\mathbf{r})$ requires the computation and storage of the double Laplacian of the single-particle wave functions, but is redundant with the density $D_{q}^{\Delta, \Delta}(\mathbf{r})$ which requires storing only the single Laplacian.

While it is inevitable that the computational requirements (both CPU time and storage) will 
augment with increasing order in gradients of the Skyrme interaction, we believe that it should not be increased more than necessary, and informed choices of local densities can help with that, especially where the derivatives of single-particle wave functions are concerned.

\section{Comparison to other schemes}

The definitions and notations proposed above in terms of Cartesian tensor densities are mnemonic, systematic and extensible, and therefore allow for expressing any local EDF of arbitrary order in gradients. To the best of our knowledge, the only other framework that offers the same features is the formulation of the local EDF in terms of spherical tensor densities proposed and employed in Refs. 21, 22, 24]. While both schemes can be equivalently used to express the same physics, using one or the other can make an enormous practical difference for formal and numerical applications. In a framework that is based on spherical tensors, the coupling and recoupling of gradients follows the rules of angularmomentum coupling. This makes formal manipulations less transparent than a Cartesian framework, and leads to numerous straightforward, but cumbersome, angularmomentum coupling coefficients that appear in the final expressions 5 When working with spherical tensors, there is a natural preference for a stretched coupling of gradient operators, as this automatically ensures that the resulting densities to be non-redundant and irreducible [21]. These densities, however, might not be the computationally most advantageous ones.

While a framework using spherical tensors can be naturally applied to systems that exhibit spherical symmetry, it can become cumbersome to use in numerical codes for deformed nuclei. One reason is that the higherrank spherical tensors inevitably combine gradients into different Cartesian directions, because of the inherent definition of spherical vector components 21. This can lead to the computational inconveniences sketched in Sect. IVA when several gradients are present in a given term. As can be deduced from Table XXI of Ref. [21], there is not always a one-to-one correspondence between the spherical tensor densities as defined there and advantageous choices for cartesian tensor densities. The spherical tensor framework of Refs. 21, 22, 24] also automatically treats the various irreducible representations of high-rank tensors as different objects, which increases the number of densities and potentials that have to be tracked in calculations for deformed

\footnotetext{
5 The relation between the definition of the coupling constants of the Cartesian formulation of the generating N2LO pseudopotential $\hat{V}_{\mathrm{N} 2 \mathrm{LO}}^{\mathrm{C}}$ of Eq. (2) that is used here and its representation in the spherical tensor framework of Refs. 21, 22, 24] can be found in Ref. [29].
}

nuclei where all irreducible representations are non-zero. In such situation it is more efficient to use the full tensor instead of its decomposition, as done in the Cartesian scheme presented above.

\section{THE N2LO ENERGY DENSITY FUNCTIONAL REVISITED}

\section{A. Towards a more efficient form of the functional}

It is now rather straightforward to enumerate a set of non-redundant, irreducible normal densities to form the particle-hole part of the EDF at N2LO and, that have definite behavior under time-reversal and are guaranteed to be real. From the considerations above, it follows that a non-redundant set of densities that contain a given number of derivatives contains at most eight different ones. Among those, however, two normal densities are always reducible, and two pair densities vanish by choice when assuming that protons and neutrons are not mixed at the level of single-particle wave functions.

There are four densities without gradients

$$
D_{q}^{1,1}(\mathbf{r}), \quad D_{q, \mu}^{1, \sigma}(\mathbf{r}), \quad \tilde{D}_{q}^{1,1}(\mathbf{r}), \quad \tilde{C}_{q}^{1,1}(\mathbf{r}),
$$

which are obviously unique as they do not contain gradient operators that could be recoupled in different ways. The current densities $C_{q}^{1,1}(\mathbf{r})$ and $C_{q, \mu}^{1, \sigma}(\mathbf{r})$ always vanish for symmetry reasons, whereas $\tilde{D}_{q, \mu}^{1, \sigma}(\mathbf{r})$ and $\tilde{C}_{q, \mu}^{1, \sigma}(\mathbf{r})$ only have to considered when mixing protons and neutrons.

There are four densities containing a single gradient operator that have to be considered

$$
C_{q, \mu}^{1, \nabla}(\mathbf{r}), \quad C_{q, \mu \nu}^{1, \nabla \sigma}(\mathbf{r}), \quad \tilde{C}_{q, \mu \nu}^{1, \nabla \sigma}(\mathbf{r}), \quad \tilde{D}_{q, \mu \nu}^{1, \nabla \sigma}(\mathbf{r}) .
$$

At this order, the densities $D_{q}^{1, \nabla}(\mathbf{r})$ and $D_{q}^{1, \nabla \sigma}(\mathbf{r})$ are reducible, whereas $\tilde{D}_{q}^{1, \nabla}(\mathbf{r})$ and $\tilde{C}_{q}^{1, \nabla}(\mathbf{r})$ only have to considered when mixing protons and neutrons.

With two gradients, we can again choose four irreducible densities

$$
D_{q, \mu \nu}^{\nabla, \nabla}(\mathbf{r}), \quad D_{q, \mu \nu \kappa}^{\nabla, \nabla \sigma}(\mathbf{r}), \quad \tilde{D}_{q, \mu \nu}^{\nabla, \nabla}(\mathbf{r}), \quad \tilde{C}_{q, \mu \nu}^{\nabla, \nabla}(\mathbf{r}) .
$$

It is implied by Eq. (106) that we construct the lower-rank densities with two gradients as contractions of these densities with a Kronecker $\delta_{\mu \nu}$, such that we altogether remain very close to the traditional choice using the densities using $\tau_{q, \mu \nu}(\mathbf{r})$ and $K_{q, \mu \nu \kappa}(\mathbf{r})$. The main difference is that the new definitions automatically eliminate the reducible contributions of the full tensor densities, see Eq. (57) for an example, thereby leading to real densities (gauge permitting for the pair densities).

At the level of three derivatives, we can write down four irreducible densities with one free gradient index

$$
C_{q, \mu}^{\Delta, \nabla}(\mathbf{r}), \quad C_{q, \mu}^{\Delta, \nabla \sigma}(\mathbf{r}), \quad \tilde{D}_{q, \mu \nu}^{\Delta, \nabla \sigma}(\mathbf{r}), \quad \tilde{C}_{q, \mu \nu}^{\Delta, \nabla \sigma}(\mathbf{r}) .
$$


At this order our choice of normal densities differs entirely from Ref. 36]. Note how we have systematically chosen the Laplacian and non-contracted gradient to act on different coordinates. Not doing so would significantly increase the number of combinations of derivatives to be calculated when constructing local densities and, even more, the corresponding term in the single-particle Hamiltonian.

Finally, with four gradients we need only scalar objects. We choose

$$
D_{q}^{\Delta, \Delta}(\mathbf{r}), \quad D_{q, \mu}^{\Delta, \Delta \sigma}(\mathbf{r}), \quad \tilde{D}_{q}^{\Delta, \Delta}(\mathbf{r}), \quad \tilde{C}_{q}^{\Delta, \Delta}(\mathbf{r}),
$$

where we have opted to recouple all contracted gradients into Laplacians, balanced between primed and unprimed coordinates.

The adjustment of the only available N2LO parametrization, SN2LO1, did not include pairing degrees of freedom 36], so we will limit ourselves in what follows to the normal densities. Our choice for these can be summarized as

$$
\begin{gathered}
\mathrm{R}_{q}=\left(D_{q}^{1,1}, D_{q, \mu}^{1, \sigma}, D_{q, \mu \nu}^{\nabla, \nabla}, C_{q, \mu \nu}^{1, \nabla \sigma}, C_{q, \mu}^{1, \nabla}, D_{q, \mu \nu \kappa}^{\nabla, \nabla \sigma},\right. \\
\left.D_{q}^{\Delta, \Delta}, C_{q, \mu \nu}^{\Delta, \nabla \sigma}, D_{q, \mu}^{\Delta, \Delta \sigma}, C_{q, \mu}^{\Delta, \nabla}\right) .
\end{gathered}
$$

As in Ref. [46], we employ the shorthand notation of a density vector when talking about all normal local densities simultaneously. We index this object with latin indices, as in $\mathrm{R}_{q, a}$, to indicate a particular density as an element of the vector. Of the different elements of Eq. (109), only $C_{q, \mu}^{\Delta, \nabla}(\mathbf{r})$ has not been discussed before. It is related to the higher-order current density $\boldsymbol{\Pi}_{q}(\mathbf{r})=$ $\mathbf{C}_{q}^{(\nabla, \nabla) \nabla}$ (r) as used in Ref. [36] as follows

$$
\begin{aligned}
C_{q, \mu}^{\Delta, \nabla}(\mathbf{r}) & =-C_{q, \mu}^{(\nabla, \nabla) \nabla}(\mathbf{r})+\frac{1}{2}\left[\Delta C_{q, \mu}^{1, \nabla}(\mathbf{r})\right] \\
& -\frac{1}{2} \sum_{\nu}\left[\nabla_{\mu} \nabla_{\nu} C_{q, \nu}^{1, \nabla}(\mathbf{r})\right],
\end{aligned}
$$

which is the analogue of Eq. (103) without spin.

We reiterate that our choice of local densities, Eq. (109), is not unique, but we believe it to be one of the choices that both considerably simplifies the formal expressions and also enormously reduces the complexity of the numerical implementation of the N2LO functional form of Ref. [36]. While we do not consider here the expressions for N2LO pairing, tensor or spin-orbit terms, we expect that the choices made here will also be optimal for these extensions of the Skyrme EDF. For terms at even higher orders in gradients, the guiding principles we discussed can easily be used to construct optimal sets of densities in such cases as well and we believe that these will be compatible with the choices made here for densities up to N2LO.

Despite these simplifications, the practical implementation of the calculation of all densities discussed here is tedious. One particular aspect is especially error-prone: the properties of all components of all densities under the symmetries conserved by the implementation. For reference, we present such symmetry relations for the case of a $3 \mathrm{D}$ coordinate-space representation such as ours in Appendix D,

We lack one more definition in order to write down the form of the functional as used for the SN2LO1 parametrization of Ref. [36] in the new notation and with the new choice of densities. As with the historical notation, we define the isoscalar and isovector densities

$$
\begin{aligned}
& D_{0}^{A, B}(\mathbf{r}) \equiv D_{n}^{A, B}(\mathbf{r})+D_{p}^{A, B}(\mathbf{r}), \\
& D_{1}^{A, B}(\mathbf{r}) \equiv D_{n}^{A, B}(\mathbf{r})-D_{p}^{A, B}(\mathbf{r}), \\
& C_{0}^{A, B}(\mathbf{r}) \equiv C_{n}^{A, B}(\mathbf{r})+C_{p}^{A, B}(\mathbf{r}), \\
& C_{1}^{A, B}(\mathbf{r}) \equiv C_{n}^{A, B}(\mathbf{r})-C_{p}^{A, B}(\mathbf{r}),
\end{aligned}
$$

and similar for the spin densities. Note, however, that the construction of isoscalar and isovector pair and spin-pair densities requires additional considerations 19, 44, 51]. In calculations that do not mix protons and neutrons as assumed here, using isovector pair densities instead of proton and neutron pair densities unnecessarily complicates the formulation of the pairing EDF and the HFB equations and will therefore not be addressed here.

\section{B. An alternative functional form of SN2LO1}

With these definitions at hand we now have all the tools to write down the LO, NLO and N2LO energy densities from Section $\amalg \mathrm{A}$ as a function of the coupling constants $A_{t, \mathrm{e} / \mathrm{o}}^{(i, j)}$ and the densities:

$$
\begin{aligned}
\mathcal{E}_{\mathrm{Sk}, \mathrm{e}}^{(0)}(\mathbf{r})= & \sum_{t=0,1}\left[A_{t, \mathrm{e}}^{(0,1)}\left(D_{t}^{1,1}\right)^{2}+A_{t, \mathrm{e}}^{(0,2)}\left(D_{0}^{1,1}\right)^{\alpha}\left(D_{t}^{1,1}\right)^{2}\right], \\
\mathcal{E}_{\mathrm{Sk}, \mathrm{e}}^{(2)}(\mathbf{r})= & \sum_{t=0,1}\left[A_{t, \mathrm{e}}^{(2,1)} D_{t}^{1,1}\left(\Delta D_{t}^{1,1}\right)+A_{t, \mathrm{e}}^{(2,2)} D_{t}^{1,1} D_{t}^{(\nabla, \nabla)}+A_{t, \mathrm{e}}^{(2,3)} \sum_{\mu \nu} C_{t, \mu \nu}^{1, \nabla \sigma} C_{t, \mu \nu}^{1, \nabla \sigma}+A_{t, \mathrm{e}}^{(2,4)} D_{t}^{1,1}\left(\nabla \cdot \mathbf{C}_{t}^{1, \nabla \times \sigma}\right)\right], \\
\mathcal{E}_{\mathrm{Sk}, \mathrm{e}}^{(4)}(\mathbf{r})=\sum_{t=0,1}\left[A_{t, \mathrm{e}}^{(4,1)}\left(\Delta D_{t}^{1,1}\right)\left(\Delta D_{t}^{1,1}\right)+A_{t, \mathrm{e}}^{(4,2)} D_{t}^{1,1} D_{t}^{\Delta, \Delta}+A_{t, \mathrm{e}}^{(4,3)} D_{t}^{(\nabla, \nabla)} D_{t}^{(\nabla, \nabla)}\right. & \\
\quad & \quad A_{t, \mathrm{e}}^{(4,4)} \sum_{\mu \nu} D_{t, \mu \nu}^{\nabla, \nabla} D_{t, \mu \nu}^{\nabla, \nabla}+A_{t, \mathrm{e}}^{(4,5)} \sum_{\mu \nu} D_{t, \mu \nu}^{\nabla, \nabla}\left(\nabla_{\mu} \nabla_{\nu} D_{t}^{1,1}\right)
\end{aligned}
$$




$$
\begin{aligned}
&\left.+A_{t, \mathrm{e}}^{(4,6)} \sum_{\mu \nu} C_{t, \mu \nu}^{1, \nabla \sigma}\left(\Delta C_{t, \mu \nu}^{1, \nabla \sigma}\right)+A_{t, \mathrm{e}}^{(4,7)} \sum_{\mu \nu \kappa}\left(\nabla_{\mu} C_{t, \mu \kappa}^{1, \nabla \sigma}\right)\left(\nabla_{\nu} C_{t, \nu \kappa}^{1, \nabla \sigma}\right)+A_{t, \mathrm{e}}^{(4,8)} \sum_{\mu \nu} C_{t, \mu \nu}^{1, \nabla \sigma} C_{t, \mu \nu}^{\Delta, \nabla \sigma}\right], \\
& \mathcal{E}_{\mathrm{Sk}, \mathrm{o}}^{(0)}(\mathbf{r})= \sum_{t=0,1}\left[A_{t, \mathrm{o}}^{(0,1)} \mathbf{D}_{t}^{1, \sigma} \cdot \mathbf{D}_{t}^{1, \sigma}+A_{t, \mathrm{o}}^{(0,2)}\left(D_{0}^{1,1}\right)^{\alpha} \mathbf{D}_{t}^{1, \sigma} \cdot \mathbf{D}_{t}^{1, \sigma}\right], \\
& \mathcal{E}_{\mathrm{Sk}, \mathrm{o}}^{(2)}(\mathbf{r})= \sum_{t=0,1}\left[A_{t, \mathrm{o}}^{(2,1)} \mathbf{D}_{t}^{1, \sigma} \cdot\left(\Delta \mathbf{D}_{t}^{1, \sigma}\right)+A_{t, \mathrm{o}}^{(2,2)} \mathbf{D}_{t}^{1, \sigma} \cdot \mathbf{D}_{t}^{(\nabla, \nabla) \sigma}+A_{t, \mathrm{o}}^{(2,3)} \mathbf{C}_{t}^{1, \nabla} \cdot \mathbf{C}_{t}^{1, \nabla}+A_{t, \mathrm{o}}^{(2,4)} \mathbf{D}_{t}^{1, \sigma} \cdot\left(\nabla \times \mathbf{C}_{t}^{1, \nabla}\right)\right], \\
& \mathcal{E}_{\mathrm{Sk}, \mathrm{o}}^{(4)}(\mathbf{r})=\sum_{t=0,1}\left[A_{t, \mathrm{o}}^{(4,1)}\left(\Delta \mathbf{D}_{t}^{1, \sigma}\right) \cdot\left(\Delta \mathbf{D}_{t}^{1, \sigma}\right)+A_{t, \mathrm{o}}^{(4,2)} \mathbf{D}_{t}^{1, \sigma} \cdot \mathbf{D}_{t}^{\Delta, \Delta \sigma}+A_{t, \mathrm{o}}^{(4,3)} \mathbf{D}_{t}^{(\nabla, \nabla) \sigma} \cdot \mathbf{D}_{t}^{(\nabla, \nabla) \sigma}\right. \\
& \quad+A_{t, \mathrm{o}}^{(4,4)} \sum_{\mu \nu \kappa} D_{\mu \nu \kappa}^{\nabla, \nabla \sigma} D_{\mu \nu \kappa}^{\nabla, \nabla \sigma}+A_{t, \mathrm{o}}^{(4,5)} \sum_{\mu \nu \kappa} D_{\mu \nu \kappa}^{\nabla, \nabla \sigma}\left(\nabla_{\mu} \nabla_{\nu} D_{\kappa}^{1, \sigma}\right) \\
&\left.\quad+A_{t, \mathrm{o}}^{(4,6)} \mathbf{C}_{t}^{1, \nabla} \cdot\left(\Delta \mathbf{C}_{t}^{1, \nabla}\right)+A_{t, \mathrm{o}}^{(4,7)}\left(\boldsymbol{\nabla} \cdot \mathbf{C}_{t}^{1, \nabla}\right)\left(\boldsymbol{\nabla} \cdot \mathbf{C}_{t}^{1, \nabla}\right)+A_{t, \mathrm{o}}^{(4,8)} \mathbf{C}_{t}^{1, \nabla} \cdot \mathbf{C}_{t}^{\Delta, \nabla}\right]
\end{aligned}
$$

where we have dropped the explicit position dependence of the densities for brevity. Other than being formulated in terms of $D$ and $C$ objects, the LO and NLO energy densities are identical to those in a traditional NLO functional. For the N2LO energy density, some additional work is required to rewrite all terms using only our choice of densities. These additional steps are sketched in Appendix $\mathrm{A}$ together with the relation between the four coupling constants $C^{(4)}$ in Eqs. (15) and the $A_{t, \mathrm{e} / \mathrm{o}}^{(4, j)}$. If one wants to link the EDF to an effective interaction, as we do here, the coupling constants $A_{t / e}^{(i, j)}$ are related to each other and the parameters of the pseudopotential. In this case, the N2LO energy density can be parameterized in terms of four numbers, $t_{1}^{(4)}, t_{2}^{(4)}, x_{1}^{(4)}$ and $x_{2}^{(4)}$. The relevant expressions for the coupling constants in terms of these parameters are given in Appendix B.

If the connection to an effective interaction is maintained, then this form of the EDF is locally gauge invariant. This in turn implies that a continuity equation relates the divergence of the current $\mathbf{j}_{q}(\mathbf{r})=\mathbf{C}_{q}^{1, \nabla}(\mathbf{r})$ to the time derivative of the density $\rho_{q}(\mathbf{r})=D_{q}^{1,1}(\mathbf{r})[\underline{56}$ ]

$$
\frac{\partial}{\partial t} D_{q}^{1,1}(\mathbf{r}, t)=-\frac{\hbar}{m} \boldsymbol{\nabla} \cdot \mathbf{C}_{q}^{1, \nabla}(\mathbf{r}, t) .
$$

Since we consider here only static calculations, Eq. (121) implies that the divergence of the current density vanishes identically, as does the penultimate term in the N2LO time-odd energy density, Eq. (120). For locally gauge invariant EDFs, another continuity equation relates $C_{q, \mu \nu}^{1, \nabla \sigma}(\mathbf{r})$ to the time derivative of $D_{q, \kappa}^{1, \sigma}(\mathbf{r})[56$

$$
\frac{\partial}{\partial t} D_{q, \kappa}^{1, \sigma}(\mathbf{r}, t)=-\frac{\hbar}{m} \sum_{\mu} \nabla_{\mu} C_{q, \mu \kappa}^{1, \nabla \sigma}(\mathbf{r}, t)
$$

such that the penultimate term of the time-even N2LO energy density, Eq. (117), vanishes as well in static calculations. We have not dropped either of those two terms from the expressions in this section and the following ones, as they will always contribute to the total energy for time-dependent approaches as well as for static calculations that use possible extensions of the functional that do not conserve local gauge invariance.

If the EDF is generated as the expectation value of a density-independent effective interaction without any further manipulation of coupling constants, then the functional is automatically self-interaction free [57, 58]. This means that the total energy of a system composed of just one particle is its kinetic energy. We have checked numerically that, when the relations between the coupling constants of Appendix B hold and the density dependence is omitted for the purpose of such test, the contribution of the Skyrme EDF to the singleparticle energy and the total energy is zero up to numerical noise. Self-interaction freedom can only be tested in a non-self-consistent calculation, where for an arbitrarily generated localized single-particle state the expectation value of the single-particle Hamiltonian and the total energy are evaluated by taking only this states' contributions into account. Performing such test requires the breaking of rotational symmetry as well as time-reversal symmetry, implying that all local densities are non-vanishing. Such calculation constitutes a powerful check that both Eqs. (115)-(120) and the expressions in Ref. [36] have correctly been derived from the generating effective interaction (2) and correctly been implemented in our code. We have also verified that, for such symmetry broken cases, the total energy calculated from the single-particle energies at self-consistency (see Ref. [45] for details) is equal to a direct integration of the energy density at the $\mathrm{keV}$ level, as is the case for standard NLO functionals.

\section{Mean-field potentials and single-particle Hamiltonian}

Using the density vector R as defined in Eq. (109) for compact notation, the expression for the energy can be rewritten as $E_{\mathrm{tot}}(\mathrm{R})$. The individual terms in the singleparticle Hamiltonian are then obtained by rewriting the 
variation of the energy with respect to the full density matrix $\rho\left(\mathbf{r}^{\prime} \sigma^{\prime}, \mathbf{r} \sigma\right)$ as the sum of variations with respect to the local densities

$$
\begin{aligned}
h_{q}\left(\mathbf{r} \sigma, \mathbf{r}^{\prime} \sigma^{\prime}\right) & \equiv \frac{\delta E_{\mathrm{tot}}}{\delta \rho\left(\mathbf{r}^{\prime} \sigma, \mathbf{r} \sigma\right)} \\
& =\sum_{a} \int d^{3} r^{\prime \prime} \frac{\delta E_{\mathrm{tot}}(\mathrm{R})}{\delta \mathrm{R}_{q, a}\left(\mathbf{r}^{\prime \prime}\right)} \frac{\delta \mathrm{R}_{q, a}\left(\mathbf{r}^{\prime \prime}\right)}{\delta \rho_{q}\left(\mathbf{r}^{\prime} \sigma^{\prime}, \mathbf{r} \sigma\right)}
\end{aligned}
$$

The single-particle Hamiltonian is a $2 \times 2$ matrix in the space of spin $1 / 2$ spinors, whose matrix elements are given by

$$
\begin{aligned}
& h_{j k}=\left\langle\Psi_{j}\left|\hat{h}_{q}\right| \Psi_{k}\right\rangle=\iint d^{3} r d^{3} r^{\prime} \sum_{\sigma \sigma^{\prime}} \psi_{j}^{*}(\mathbf{r} \sigma) h_{q}\left(\mathbf{r} \sigma, \mathbf{r}^{\prime} \sigma^{\prime}\right) \psi_{k}\left(\mathbf{r}^{\prime} \sigma^{\prime}\right) \\
& =\iint d^{3} r d^{3} r^{\prime}\left(\psi_{j}^{*}(\mathbf{r}+), \psi_{j}^{*}(\mathbf{r}-)\right)\left(\begin{array}{cc}
h_{q}\left(\mathbf{r}+, \mathbf{r}^{\prime}+\right) & h_{q}\left(\mathbf{r}+, \mathbf{r}^{\prime}-\right) \\
h_{q}\left(\mathbf{r}-, \mathbf{r}^{\prime}+\right) & h_{q}\left(\mathbf{r}-, \mathbf{r}^{\prime}-\right)
\end{array}\right)\left(\begin{array}{c}
\psi_{k}\left(\mathbf{r}^{\prime}+\right) \\
\psi_{k}\left(\mathbf{r}^{\prime}-\right)
\end{array}\right),
\end{aligned}
$$

where we assume that the single-particle states $j$ and $k$ are of the same nucleon species $q$. The derivative of the energy with respect to the density $\mathrm{R}_{q, a}(\mathbf{r})$ can be identified as an associated mean-field potential $\mathrm{F}_{q, a}(\mathbf{r})$, defined as

$$
\mathrm{F}_{q, a}(\mathbf{r}) \equiv \frac{\delta E_{\mathrm{tot}}(\mathrm{R})}{\delta \mathrm{R}_{q, a}(\mathbf{r})} .
$$

Each potential $F_{q, a}(\mathbf{r})$ depends in general on the density vectors $\mathrm{R}_{p}(\mathbf{r})$ and $\mathrm{R}_{n}(\mathbf{r})$ of both nucleon species.

We use the letters $F$ and $G$ to distinguish between potentials corresponding to $D$ and $C$ densities, respectively. As there are four generic types of local densities and currents, there are four different types of generic potentials that are given by

$$
\begin{aligned}
F_{q}^{A B}\left(\mathbf{r}^{\prime \prime}\right) & \equiv \frac{\delta E_{\mathrm{tot}}}{\delta D_{q}^{A B}\left(\mathbf{r}^{\prime \prime}\right)}=\int d^{3} r^{\prime \prime \prime \prime} \frac{\delta \mathcal{E}\left(\mathbf{r}^{\prime \prime \prime \prime}\right)}{\delta D_{q}^{A B}\left(\mathbf{r}^{\prime \prime}\right)}, \\
G_{q}^{A B}\left(\mathbf{r}^{\prime \prime}\right) & \equiv \frac{\delta E_{\mathrm{tot}}}{\delta C_{q}^{A B}\left(\mathbf{r}^{\prime \prime}\right)}=\int d^{3} r^{\prime \prime \prime \prime \prime} \frac{\delta \mathcal{E}\left(\mathbf{r}^{\prime \prime \prime \prime}\right)}{\delta C_{q}^{A B}\left(\mathbf{r}^{\prime \prime}\right)}, \\
F_{q}^{A B \sigma}\left(\mathbf{r}^{\prime \prime}\right) & \equiv \frac{\delta E_{\mathrm{tot}}}{\delta D_{q}^{A B \sigma}\left(\mathbf{r}^{\prime \prime}\right)}=\int d^{3} r^{\prime \prime \prime \prime \prime} \frac{\delta \mathcal{E}\left(\mathbf{r}^{\prime \prime \prime \prime}\right)}{\delta D_{q}^{A B \sigma}\left(\mathbf{r}^{\prime \prime}\right)}, \\
G_{q}^{A B \sigma}\left(\mathbf{r}^{\prime \prime}\right) & \equiv \frac{\delta E_{\mathrm{tot}}}{\delta C_{q}^{A B \sigma}\left(\mathbf{r}^{\prime \prime}\right)}=\int d^{3} r^{\prime \prime \prime \prime} \frac{\delta \mathcal{E}\left(\mathbf{r}^{\prime \prime \prime \prime}\right)}{\delta C_{q}^{A B \sigma}\left(\mathbf{r}^{\prime \prime}\right)}
\end{aligned}
$$

The cartesian tensor structure, behavior under index exchange and symmetry properties carry over directly from a local density to its associated potential. The identification of potentials (126) - (129) with the existing literature is analogous to the mapping of the densities outlined in Sec. IVA for example $F_{q}^{1,1}(\mathbf{r})$ corresponds to the central potential that is commonly called $U_{q}(\mathbf{r})$.

Similar to the density vector $\mathrm{R}_{q}(\mathbf{r})$, we define the potential vector $F_{q}(\mathbf{r})$ that is composed of all the meanfield potentials

$$
\begin{gathered}
\mathrm{F}_{q}=\left\{F_{q}^{1,1}, F_{q, \mu}^{1, \sigma}, F_{q, \mu \nu}^{\nabla, \nabla}, G_{q, \mu \nu}^{1, \nabla \sigma}, G_{q}^{1, \nabla}, F_{q, \mu \nu \kappa}^{\nabla, \nabla \sigma},\right. \\
\left.F_{q}^{\Delta, \Delta}, G_{q, \mu \nu}^{\Delta, \nabla \sigma}, F_{q, \mu}^{\Delta, \Delta \sigma}, G_{q, \mu}^{\Delta, \nabla}\right\} .
\end{gathered}
$$

The contribution to the single-particle Hamiltonian from the term containing derivatives with respect to $D_{q}^{A B}\left(\mathbf{r}^{\prime \prime}\right)$ in the chain rule when deriving the energy with respect to $\rho_{q}\left(\mathbf{r}^{\prime} \sigma^{\prime}, \mathbf{r} \sigma\right)$ is

$$
\begin{aligned}
\int d^{3} r^{\prime \prime} & \frac{\delta D_{q}^{A B}\left(\mathbf{r}^{\prime \prime}\right)}{\delta \rho_{q}\left(\mathbf{r}^{\prime} \sigma^{\prime}, \mathbf{r} \sigma\right)} \frac{\delta E_{\mathrm{tot}}}{\delta D_{q}^{A B}\left(\mathbf{r}^{\prime \prime}\right)} \\
= & \int d^{3} r^{\prime \prime} \frac{1}{2}\left\{\left[\hat{A}^{\prime \prime} \delta_{\mathbf{r}^{\prime \prime} \mathbf{r}}\right] F_{q}^{A B}\left(\mathbf{r}^{\prime \prime}\right)\left[\hat{B}^{\prime \prime} \delta_{\mathbf{r}^{\prime \prime} \mathbf{r}^{\prime}}\right]\right. \\
& \left.+\left[\hat{B}^{\prime \prime} \delta_{\mathbf{r}^{\prime \prime} \mathbf{r}}\right] F_{q}^{A B}\left(\mathbf{r}^{\prime \prime}\right)\left[\hat{A}^{\prime \prime} \delta_{\mathbf{r}^{\prime \prime} \mathbf{r}^{\prime}}\right]\right\} \delta_{\sigma \sigma^{\prime}}
\end{aligned}
$$

In the matrix element (124), $\left[\hat{A}^{\prime \prime} \delta_{\mathbf{r}^{\prime \prime} \mathbf{r}}\right]$ and $\left[\hat{B}^{\prime \prime} \delta_{\mathbf{r}^{\prime \prime} \mathbf{r}}\right]$ become "gradient operators acting to the left" on the single-particle state with coordinate $\mathbf{r}$, whereas $\left[\hat{B}^{\prime \prime} \delta_{\mathbf{r}^{\prime \prime} \mathbf{r}^{\prime}}\right]$ and $\left[\hat{A}^{\prime \prime} \delta_{\mathbf{r}^{\prime \prime} \mathbf{r}^{\prime}}\right]$ are "gradient operators acting to the right" on the single-particle state with coordinate $\mathbf{r}^{\prime}$. Through integration by parts, however, the gradients acting on the wave function on one side can be transferred to act on the potential $F_{q}^{A B}\left(\mathbf{r}^{\prime \prime}\right)$ times the wave function on the other side.

The latter is a necessity when one is interested in the action of the single-particle Hamiltonian on a state that is not its eigenstate, which is an ingredient of many schemes for the iterative diagonalization of the singleparticle Hamiltonian, such as the heavy-ball method used by us [4]. This is the form into which we will bring the expression for $h_{q}\left(\mathbf{r} \sigma, \mathbf{r}^{\prime} \sigma^{\prime}\right)$. In general, one finds 


$$
\begin{aligned}
& \int d^{3} r^{\prime \prime} \frac{\delta D_{q}^{A B}\left(\mathbf{r}^{\prime \prime}\right)}{\delta \rho_{q}\left(\mathbf{r}^{\prime} \sigma^{\prime}, \mathbf{r} \sigma^{\prime}\right)} \frac{\delta E_{\mathrm{tot}}}{\delta D_{q}^{A B}\left(\mathbf{r}^{\prime \prime}\right)}=+\frac{1}{2}\left((-)^{n_{A}} \hat{A}\left\{F_{q}^{A B}(\mathbf{r})\left[\hat{B} \delta_{\mathbf{r r}^{\prime}}\right]\right\}+(-)^{n_{B}} \hat{B}\left\{F_{q}^{A B}(\mathbf{r})\left[\hat{A} \delta_{\mathbf{r} \mathbf{r}^{\prime}}\right]\right\}\right) \delta_{\sigma \sigma^{\prime}}, \\
& \int d^{3} r^{\prime \prime} \frac{\delta C_{q}^{A B}\left(\mathbf{r}^{\prime \prime}\right)}{\delta \rho_{q}\left(\mathbf{r}^{\prime} \sigma^{\prime}, \mathbf{r} \sigma\right)} \frac{\delta E_{\mathrm{tot}}}{\delta C_{q}^{A B}\left(\mathbf{r}^{\prime \prime}\right)}=-\frac{i}{2}\left((-)^{n_{A}} \hat{A}\left\{G_{q}^{A B}(\mathbf{r})\left[\hat{B} \delta_{\mathbf{r r}^{\prime}}\right]\right\}-(-)^{n_{B}} \hat{B}\left\{G_{q}^{A B}(\mathbf{r})\left[\hat{A} \delta_{\mathbf{r r}^{\prime}}\right]\right\}\right) \delta_{\sigma \sigma^{\prime}}, \\
& \int d^{3} r^{\prime \prime} \frac{\delta D_{q}^{A B \sigma}\left(\mathbf{r}^{\prime \prime}\right)}{\delta \rho_{q}\left(\mathbf{r}^{\prime} \sigma^{\prime}, \mathbf{r} \sigma\right)} \frac{\delta E_{\mathrm{tot}}}{\delta D_{q}^{A B \sigma}\left(\mathbf{r}^{\prime \prime}\right)}=+\frac{1}{2}\left((-)^{n_{A}} \hat{A}\left\{F_{q}^{A B \sigma}(\mathbf{r})\left[\hat{B} \delta_{\mathbf{r r}^{\prime}}\right]\right\}+(-)^{n_{B}} \hat{B}\left\{F_{q}^{A B \sigma}(\mathbf{r})\left[\hat{A} \delta_{\mathbf{r} \mathbf{r}^{\prime}}\right]\right\}\right)\left\langle\sigma|\hat{\sigma}| \sigma^{\prime}\right\rangle, \\
& \int d^{3} r^{\prime \prime} \frac{\delta C_{q}^{A B \sigma}\left(\mathbf{r}^{\prime \prime}\right)}{\delta \rho_{q}\left(\mathbf{r} \sigma, \mathbf{r}^{\prime} \sigma^{\prime}\right)} \frac{\delta E_{\mathrm{tot}}}{\delta C_{q}^{A B \sigma}\left(\mathbf{r}^{\prime \prime}\right)}=-\frac{\mathrm{i}}{2}\left((-)^{n_{A}} \hat{A}\left\{G_{q}^{A B \sigma}(\mathbf{r})\left[\hat{B} \delta_{\mathbf{r r}^{\prime}}\right]\right\}-(-)^{n_{B}} \hat{B}\left\{G_{q}^{A B \sigma}(\mathbf{r})\left[\hat{A} \delta_{\mathbf{r r}^{\prime}}\right]\right\}\right)\left\langle\sigma|\hat{\sigma}| \sigma^{\prime}\right\rangle,
\end{aligned}
$$

where $n_{A}$ and $n_{B}$ are the order in gradient operators of $\hat{A}$ and $\hat{B}$, respectively. Using these generic expressions, the calculation of the single-particle Hamiltonian can be automatized, both for its matrix elements as well as for its action on a given single-particle state.

The considerations concerning the often significant differences in computational cost of alternative choices for local densities sketched in Sec. IIID2 also apply to the numerical application of the contributions (132)(135) to the single-particle Hamiltonian: for reasons of storage and computational time, it is advantageous to couple higher-order derivatives to Laplacians whenever possible, and to use a symmetric form where $\hat{A}=\hat{B}$ whenever possible for $D_{q}^{A B}(\mathbf{r})$ and $D_{q}^{A B \sigma}(\mathbf{r})$, such that the two terms in Eqs. (132) and (134) can be combined into one. For non-redundant $C_{q}^{A B}(\mathbf{r})$ and $C_{q}^{A B \sigma}(\mathbf{r})$ this cannot be done, but at least for many of the widely-used locally gauge-invariant EDFs there are choices for which $\hat{A}$ and/or $\hat{B}$ acting on either $G_{q}^{A B}(\mathbf{r})$ and/or $G_{q}^{A B \sigma}(\mathbf{r})$ gives zero when the expressions for these potentials are proportional to the right-hand side of a continuity equation such as Eqs. (121) or (122), see for example
Ref. [11]. There also is a natural preference to express the EDF through local densities and currents that enter the expectation values of one-body operators that are frequently used to formulate constraints on shape degrees of freedom, angular momentum, etc, such that these additional terms do not introduce additional operator structures in the resulting single-particle Hamiltonian.

The single-particle Hamiltonian obtained from the N2LO EDF (115)-1120 naturally breaks down into ten parts

$$
\begin{aligned}
h_{q}\left(\mathbf{r} \sigma, \mathbf{r}^{\prime} \sigma^{\prime}\right) & =h_{q, \mathrm{e}}^{(0)}\left(\mathbf{r} \sigma, \mathbf{r}^{\prime} \sigma^{\prime}\right)+h_{q, \mathrm{o}}^{(0)}\left(\mathbf{r} \sigma, \mathbf{r}^{\prime} \sigma^{\prime}\right) \\
& +h_{q, \mathrm{e}}^{(1)}\left(\mathbf{r} \sigma, \mathbf{r}^{\prime} \sigma^{\prime}\right)+h_{q, \mathrm{o}}^{(1)}\left(\mathbf{r} \sigma, \mathbf{r}^{\prime} \sigma^{\prime}\right) \\
& +h_{q, \mathrm{e}}^{(2)}\left(\mathbf{r} \sigma, \mathbf{r}^{\prime} \sigma^{\prime}\right)+h_{q, \mathrm{o}}^{(2)}\left(\mathbf{r} \sigma, \mathbf{r}^{\prime} \sigma^{\prime}\right) \\
& +h_{q, \mathrm{e}}^{(3)}\left(\mathbf{r} \sigma, \mathbf{r}^{\prime} \sigma^{\prime}\right)+h_{q, \mathrm{o}}^{(3)}\left(\mathbf{r} \sigma, \mathbf{r}^{\prime} \sigma^{\prime}\right) \\
& +h_{q, \mathrm{e}}^{(4)}\left(\mathbf{r} \sigma, \mathbf{r}^{\prime} \sigma^{\prime}\right)+h_{q, \mathrm{o}}^{(4)}\left(\mathbf{r} \sigma, \mathbf{r}^{\prime} \sigma^{\prime}\right)
\end{aligned}
$$

which are time-even and time-odd structures with up to four gradients acting on the single-particle states

$$
\begin{aligned}
& h_{q, \mathrm{e}}^{(0)}\left(\mathbf{r} \sigma, \mathbf{r}^{\prime} \sigma^{\prime}\right)=\delta_{\sigma \sigma^{\prime}} F_{q}^{1,1}(\mathbf{r}) \delta_{\mathbf{r} \mathbf{r}^{\prime}}, \\
& h_{q, \mathrm{o}}^{(0)}\left(\mathbf{r} \sigma, \mathbf{r}^{\prime} \sigma^{\prime}\right)=\sum_{\mu}\left\langle\sigma\left|\hat{\sigma}_{\mu}\right| \sigma^{\prime}\right\rangle F_{q, \mu}^{1, \sigma}(\mathbf{r}) \delta_{\mathbf{r r}^{\prime}}, \\
& h_{q, \mathrm{e}}^{(1)}\left(\mathbf{r} \sigma, \mathbf{r}^{\prime} \sigma^{\prime}\right)=-\frac{i}{2} \sum_{\mu \nu}\left\langle\sigma\left|\hat{\sigma}_{\nu}\right| \sigma^{\prime}\right\rangle\left[\nabla_{\mu} G_{q, \mu \nu}^{1, \nabla \sigma}(\mathbf{r})+G_{q, \mu \nu}^{1, \nabla \sigma}(\mathbf{r}) \nabla_{\mu}\right] \delta_{\mathbf{r r}^{\prime}}, \\
& h_{q, \mathrm{o}}^{(1)}\left(\mathbf{r} \sigma, \mathbf{r}^{\prime} \sigma^{\prime}\right)=-\frac{i}{2} \delta_{\sigma \sigma^{\prime}} \sum_{\mu}\left[\nabla_{\mu} G_{q, \mu}^{1, \nabla}(\mathbf{r})+G_{q, \mu}^{1, \nabla}(\mathbf{r}) \nabla_{\mu}\right] \delta_{\mathbf{r r} \mathbf{r}^{\prime}}, \\
& h_{q, \mathrm{e}}^{(2)}\left(\mathbf{r} \sigma, \mathbf{r}^{\prime} \sigma^{\prime}\right)=-\delta_{\sigma \sigma^{\prime}} \sum_{\mu \nu} \nabla_{\mu} F_{q, \mu \nu}^{\nabla, \nabla}(\mathbf{r}) \nabla_{\nu} \delta_{\mathbf{r}^{\prime} \mathbf{r}}, \\
& h_{q, \mathrm{o}}^{(2)}\left(\mathbf{r} \sigma, \mathbf{r}^{\prime} \sigma^{\prime}\right)=-\sum_{\mu \nu \kappa}\left\langle\sigma\left|\hat{\sigma}_{\kappa}\right| \sigma^{\prime}\right\rangle \nabla_{\mu} F_{q, \mu \nu \kappa}^{\nabla, \nabla \sigma}(\mathbf{r}) \nabla_{\nu} \delta_{\mathbf{r r}^{\prime}}, \\
& h_{q, \mathrm{e}}^{(3)}\left(\mathbf{r} \sigma, \mathbf{r}^{\prime} \sigma^{\prime}\right)=-\frac{i}{2} \sum_{\mu \nu}\left\langle\sigma\left|\hat{\sigma}_{\nu}\right| \sigma^{\prime}\right\rangle\left[\nabla_{\mu} G_{q, \mu \nu}^{\Delta, \nabla \sigma}(\mathbf{r}) \Delta+\Delta G_{q, \mu \nu}^{\Delta, \nabla \sigma}(\mathbf{r}) \nabla_{\mu}\right] \delta_{\mathbf{r r}^{\prime}}, \\
& h_{q, \mathrm{o}}^{(3)}\left(\mathbf{r} \sigma, \mathbf{r}^{\prime} \sigma^{\prime}\right)=-\frac{i}{2} \delta_{\sigma \sigma^{\prime}} \sum_{\mu}\left[\nabla_{\mu} G_{q, \mu}^{\Delta, \nabla}(\mathbf{r}) \Delta+\Delta G_{q, \mu}^{\Delta, \nabla}(\mathbf{r}) \nabla_{\mu}\right] \delta_{\mathbf{r r}^{\prime}},
\end{aligned}
$$




$$
\begin{aligned}
& h_{q, \mathrm{e}}^{(4)}\left(\mathbf{r} \sigma, \mathbf{r}^{\prime} \sigma^{\prime}\right)=\delta_{\sigma \sigma^{\prime}} \Delta F_{q}^{\Delta, \Delta}(\mathbf{r}) \Delta \delta_{\mathbf{r r}} \\
& h_{q, \mathrm{o}}^{(4)}\left(\mathbf{r} \sigma, \mathbf{r}^{\prime} \sigma^{\prime}\right)=\sum_{\mu}\left\langle\sigma\left|\hat{\sigma}_{\mu}\right| \sigma^{\prime}\right\rangle \Delta F_{q, \mu}^{\Delta, \Delta \sigma}(\mathbf{r}) \Delta \delta_{\mathbf{r r}^{\prime}} .
\end{aligned}
$$

The reader is advised that there is an implicit product rule in Eqs. (139)-(146): the gradients act on everything to their right. However, $\hat{h}_{q}\left(\mathbf{r} \sigma, \mathbf{r}^{\prime} \sigma^{\prime}\right)$ is usually used such that there is no other function depending on the position $\mathbf{r}$ other than the potentials and the delta function $\delta_{\mathbf{r r}^{\prime}}$. In that sense, the presence of $\delta_{\mathbf{r r}^{\prime}}$ keeps the derivatives "inside" of $\hat{h}_{q}\left(\mathbf{r} \sigma, \mathbf{r}^{\prime} \sigma^{\prime}\right)$. The integration over $d^{3} r^{\prime}$ in a matrix element of $\hat{h}_{q}$ such as (124) then transfers the derivatives to whatever function of the position $\mathbf{r}$ that is under the integral. With this, all contributions from the local Skyrme EDF (meaning that it only depends on densities that are all evaluated at the same position r) lead to local terms in the single-particle Hamiltonian as expected. While the direct term of the Coulomb energy is a non-local functional of local densities, it nevertheless also leads to a local contribution to the single-particle Hamiltonian. The Coulomb exchange energy in Slater approximation is again a local functional of local densities like the Skyrme interaction [1, 45], such that it also leads to a local contribution to the single-particle Hamiltonian. Further details on these standard terms that we do not address here can be found for example in Ref. [45]. However, when using the exact Coulomb exchange energy instead of the Slater approximation, one would have to deal with a non-local functional of non-local densities that inevitably leads to a manifestly non-local contribution to $h_{q}\left(\mathbf{r} \sigma, \mathbf{r}^{\prime} \sigma^{\prime}\right)$, both resulting in a substantial increase of computational time.

For completeness' sake, we include the expressions for all potentials $\mathrm{F}_{q, a}(\mathbf{r})$ obtained from the N2LO functional defined through (115)-120) in Appendix C.

The single-particle Hamiltonian is a $2 \times 2$ matrix in the space of single-particle spinors; hence, it can be decomposed into the complete set of four linearly independent complex $2 \times 2$ matrices, which can be chosen to be the union of the unit matrix $\hat{1}_{\sigma}$ and the Pauli spin matrices

$$
\hat{h}_{q}\left(\mathbf{r}, \mathbf{r}^{\prime}\right)=\hat{h}_{q, 1}\left(\mathbf{r}, \mathbf{r}^{\prime}\right) \hat{1}_{\sigma}+\hat{\mathbf{h}}_{q, \sigma}\left(\mathbf{r}, \mathbf{r}^{\prime}\right) \cdot \hat{\boldsymbol{\sigma}},
$$

The terms in Eqs. 137-146 containing $\delta_{\sigma \sigma^{\prime}}$ are the coordinate-space representation of $\hat{h}_{q, 1} \hat{1}_{\sigma}$, whereas those containing $\left\langle\sigma\left|\hat{\sigma}_{\mu}\right| \sigma^{\prime}\right\rangle$ are the coordinate-space representation of $\hat{\mathbf{h}}_{q, \sigma} \cdot \hat{\boldsymbol{\sigma}}$ in this decomposition. The former are related to variation with respect to $D_{q}^{A B}(\mathbf{r})$ and $C_{q}^{A B}(\mathbf{r})$, whereas the latter are obtained from variation with respect to $D_{q}^{A B \sigma}(\mathbf{r})$ and $C_{q}^{A B \sigma}(\mathbf{r})$. When working with irreducible non-redundant local densities, time-even (time-odd) terms in $\hat{h}_{q, 1}$ have an even (odd) number of gradients, whereas for $\hat{h}_{q, \sigma}$ it is the opposite.
It has to be stressed that there is no unequivocal relation between the time-even and time-odd LO, NLO, and N2LO parts of the EDF and the terms with a given number of gradients in the single-particle Hamiltonian (136). The reason is that the order $\ell$ of terms in $h_{q, \eta}^{(\ell)}\left(\mathbf{r} \sigma, \mathbf{r}^{\prime} \sigma^{\prime}\right), \eta=\mathrm{e}, \mathrm{o}$, is determined by the number of internal gradients in the definition of the local density whose variation generates the respective term in Eq. (123). While the terms with three and four gradients in $h_{q}\left(\mathbf{r} \sigma, \mathbf{r}^{\prime} \sigma^{\prime}\right)$ are necessarily generated by the N2LO terms $\mathcal{E}_{\mathrm{Sk}, \eta}^{(4)}(\mathbf{r})$, in the EDF, the N2LO terms also contribute to all lower-order parts of the single-particle Hamiltonian. Similarly, the NLO terms $\mathcal{E}_{\mathrm{Sk}, \eta}^{(2)}$ contribute to all terms in the single-particle Hamiltonian with up to two gradients. Only the LO terms are necessarily limited to the gradientless terms in $\hat{h}_{q}$. In addition, the density-dependent time-odd terms from Eq. (118) also contribute to the time-even part $h_{q, \mathrm{e}}^{(0)}\left(\mathbf{r} \sigma, \mathbf{r}^{\prime} \sigma^{\prime}\right)$ of the single-particle Hamiltonian, but of course only for timereversal-invariance breaking many-body states.

For some contributions to the single-particle Hamiltonian, considering N2LO terms makes the tensor structure of these terms more complex. For NLO functionals, only the scalar contribution to $F_{q, \mu \nu}^{\nabla, \nabla}(\mathbf{r})$ is needed, leading to an effective mass that is a scalar. It is only in the presence of N2LO terms, that $F_{q, \mu \nu}^{\nabla, \nabla}(\mathbf{r})$ becomes a rank-2 tensor. Similarly, for standard NLO terms without explicit tensor interaction, only the components of $F_{q, \mu \nu \kappa}^{\nabla, \nabla \sigma}(\mathbf{r})$ with $\mu=\nu$ are non-zero, leading to a spin effective mass that is a vector. In the presence of genuine NLO tensor forces, there is an additional contribution for which the elements with $\mu=\kappa$ are also non-zero, and which is traditionally treated as a separate term in the single-particle Hamiltonian [11]. For EDFs including the N2LO terms of Ref. [36], however, the potential $F_{q, \mu \nu \kappa}^{\nabla, \nabla \sigma}(\mathbf{r})$ necessarily becomes a full rank-3 tensor.

\section{The pairing energy density functional}

Like the majority of studies based on the Skyrme interaction, we use a simple pairing energy functional with parameters that are independent from those that define the particle-hole part of the pairing EDF. Its actual form is the one of a gradientless density-dependent contact pairing interaction of surface type [11, [59, 60]. Its 
gauge-invariant representation takes the form

$$
\begin{gathered}
E_{\text {pair }}=\int d^{3} r \sum_{q=p, n} \frac{V_{q}}{4}\left[1-\frac{\rho_{0}(\mathbf{r})}{\rho_{c}}\right] \tilde{\rho}_{q}(\mathbf{r}) \tilde{\rho}_{q}^{*}(\mathbf{r}) \\
=\int d^{3} r \sum_{q=p, n} \frac{V_{q}}{4}\left[1-\frac{\rho_{0}(\mathbf{r})}{\rho_{c}}\right]\left\{\tilde{D}_{q}^{1,1}(\mathbf{r}) \tilde{D}_{q}^{1,1 *}(\mathbf{r})\right. \\
\left.+\tilde{C}_{q}^{1,1}(\mathbf{r}) \tilde{C}_{q}^{1,1 *}(\mathbf{r})\right\}
\end{gathered}
$$

where the $V_{q}$ and $\rho_{c}$ are the parameters of the pairing interaction. As the single-reference EDF is real by construction, its pairing part necessarily consists of symmetrized products of pair densities and the complex conjugates of pair densities, such that only products of either two real parts or products of two imaginary parts contribute, but no cross terms between the real and imaginary parts of the various densities. Even when working in a gauge where these densities are complex, expressing the pairing EDF through $\tilde{D}_{q}^{1,1}(\mathbf{r})$ and $\tilde{C}_{q}^{1,1}(\mathbf{r})$ clearly separates terms that are constructed from densities with different spatial symmetries, cf. the discussion of Tables [V] and $\mathrm{V}$ in Appendix D.

The adaptation to the new notation of possible higherorder terms, either in gradients 44] or in the number of densities [19, 20], in the pairing EDF follows the same principles but leads to lengthy expressions that will be detailed elsewhere.

Local pairing EDFs such as Eq. (148), which are generated by contact pairing interactions, have to be regularized in one way or another in order to suppress the divergence of the pairing energy with increasing basis size. For the calculations presented in Sec. $\mathrm{V}$ this has been done by introducing a state-dependent cutoff as done earlier in Refs. [46, 59 61]. In practice, this is achieved by multiplying the two-body wave functions $\tilde{\varrho}_{j k}\left(\mathbf{r}, \mathbf{r}^{\prime}\right)$ and $\tilde{\varsigma}_{j k, \mu}\left(\mathbf{r}, \mathbf{r}^{\prime}\right)$ with state-dependent cutoff factors, that is by making the substitutions

$$
\begin{aligned}
\tilde{\varrho}_{j k}\left(\mathbf{r}, \mathbf{r}^{\prime}\right) & \rightarrow f_{j} f_{k} \tilde{\varrho}_{j k}\left(\mathbf{r}, \mathbf{r}^{\prime}\right), \\
\tilde{\varsigma}_{j k, \mu}\left(\mathbf{r}, \mathbf{r}^{\prime}\right) & \rightarrow f_{j} f_{k} \tilde{\varsigma}_{j k, \mu}\left(\mathbf{r}, \mathbf{r}^{\prime}\right) .
\end{aligned}
$$

when summing up the pair densities (75)-78). For the HFB calculations reported in Sec. $\mathrm{V}$, we use cutoffs $f_{m}$ that (i) are defined in the "Hartree-Fock basis" that diagonalizes the single-particle Hamiltonian and (ii) depend on the distance of the given single-particle level from the Fermi energy, see Refs. 45, 46, 59 61] for details. Note that the cutoff should only be introduced in the pair densities used to calculate the pairing EDF and the associated pair fields. All non-energetic observables should still be calculated with unmodified pair densities instead.

\section{E. Pairing fields}

The HFB equation is given by

$$
\left(\begin{array}{cc}
h-\lambda & \Delta \\
-\Delta^{*} & -h^{*}+\lambda
\end{array}\right)\left(\begin{array}{c}
U_{k} \\
V_{k}
\end{array}\right)=E_{k}\left(\begin{array}{l}
U_{k} \\
V_{k}
\end{array}\right),
$$

where $\lambda$ is a Lagrange multiplier for the adjustment of the average particle number [2], and

$$
\begin{aligned}
& \Delta_{j k} \equiv \frac{\delta E_{\mathrm{pair}}}{\delta \kappa_{j k}^{*}}, \\
& \Delta_{j k}^{*} \equiv \frac{\delta E_{\mathrm{pair}}}{\delta \kappa_{j k}} .
\end{aligned}
$$

When deriving the HFB equation, it is assumed that $\kappa_{j k}=-\kappa_{k j}$ and $\kappa_{j k}^{*}=-\kappa_{k j}^{*}$ are independent degrees of freedom that are varied separately [2]. For this reason, the distinction between pair densities and their complex conjugate should be formally kept even when they can be chosen to be real. In such case all pair densities without complex conjugation entering the EDF contain only $\kappa$, while all densities with explicit complex conjugation contain only $\kappa^{*}$.

Defining, analogously to Eq. (109), $\tilde{\mathrm{R}}_{q}(\mathbf{r})$ as the vector of all pair densities entering a given pairing EDF and $\tilde{\mathrm{R}}_{q}^{*}(\mathbf{r})$ as the vector of their complex conjugates, the matrix elements $\Delta_{j k}$ and $\Delta_{j k}^{*}$ can be calculated as

$$
\begin{aligned}
& \Delta_{j k}=\frac{\delta E_{\mathrm{tot}}}{\delta \kappa_{j k}^{*}}=\int d^{3} r \sum_{a} \frac{\delta E_{\mathrm{tot}}}{\delta \tilde{\mathrm{R}}_{q, a}^{*}(\mathbf{r})} \frac{\delta \tilde{\mathrm{R}}_{q, a}^{*}(\mathbf{r})}{\delta \kappa_{j k}^{*}}, \\
& \Delta_{j k}^{*}=\frac{\delta E_{\mathrm{tot}}}{\delta \kappa_{j k}}=\int d^{3} r \sum_{a} \frac{\delta E_{\mathrm{tot}}}{\delta \tilde{\mathrm{R}}_{q, a}(\mathbf{r})} \frac{\delta \tilde{\mathrm{R}}_{q, a}(\mathbf{r})}{\delta \kappa_{j k}} .
\end{aligned}
$$

Analogously to the mean-field potentials defined for the single-particle Hamiltonian, we define pair fields as

$$
\begin{aligned}
\tilde{\mathrm{F}}_{q, a}(\mathbf{r}) & \equiv \frac{\delta E_{\mathrm{tot}}}{\delta \mathrm{R}_{q, a}^{*}(\mathbf{r})}, \\
\tilde{\mathrm{F}}_{q, a}^{*}(\mathbf{r}) & \equiv \frac{\delta E_{\mathrm{tot}}}{\delta \mathrm{R}_{q, a}(\mathbf{r})} .
\end{aligned}
$$

As before, we use $\tilde{F}, \tilde{G}, \tilde{F}^{*}$ and $\tilde{G}^{*}$ to distinguish between potentials corresponding to $\tilde{D}^{*}, \tilde{C}^{*}, \tilde{D}$ and $\tilde{C}$ densities, leading to eight different types of generic pairing potentials. For pair densities without spin, these are

$$
\begin{aligned}
\tilde{F}_{q}^{A, B}(\mathbf{r}) & \equiv \frac{\delta E_{\mathrm{tot}}}{\delta D_{q}^{A, B *}(\mathbf{r})}, \\
\tilde{F}_{q}^{A, B *}(\mathbf{r}) & \equiv \frac{\delta E_{\mathrm{tot}}}{\delta D_{q}^{A, B}(\mathbf{r})}, \\
\tilde{G}_{q}^{A, B}(\mathbf{r}) & \equiv \frac{\delta E_{\mathrm{tot}}}{\delta C_{q}^{A, B *}(\mathbf{r})}, \\
\tilde{G}_{q}^{A, B *}(\mathbf{r}) & \equiv \frac{\delta E_{\mathrm{tot}}}{\delta C_{q}^{A, B}(\mathbf{r})}
\end{aligned}
$$


For local pair densities with spin, we define $\tilde{F}_{q}^{A, B \sigma}(\mathbf{r})$, $\tilde{G}_{q}^{A, B \sigma}(\mathbf{r}), \tilde{F}_{q}^{A, B \sigma *}(\mathbf{r})$ and $\tilde{G}_{q}^{A, B \sigma *}(\mathbf{r})$ analogously. For the simple pairing functional used here, Eq. (148), only four such potentials $\left(\tilde{F}_{q}^{1,1}(\mathbf{r}), \tilde{F}_{q}^{1,1 *}(\mathbf{r}), \tilde{G}_{q}^{1,1}(\mathbf{r})\right.$ and $\left.\tilde{G}_{q}^{1,1 *}(\mathbf{r})\right)$ are relevant.

In the two-basis-method [46, 61] we employ to solve the HFB equation, only the matrix elements $\Delta_{j k}$ and $\Delta_{j k}^{*}$ in a basis of limited size are needed at each iteration instead of the action of the full pair Hamiltonian on some quasiparticle wave function. In this case, the calculation of contributions from densities at arbitrary order in gradients from Eqs. (75)-(78) is straightforward and requires the construction of the spatial functions

$$
\begin{aligned}
\frac{\delta \tilde{D}_{q}^{A, B}(\mathbf{r})}{\delta \kappa_{j k}} & =\frac{1}{2}\left(\hat{A}^{\prime} \hat{B}+\hat{A} \hat{B}^{\prime}\right) \operatorname{Re}\left\{\tilde{\varrho}_{k j}\left(\mathbf{r}, \mathbf{r}^{\prime}\right)-\tilde{\varrho}_{j k}(\mathbf{r}, \mathbf{r}\right. \\
& =\left.\left(\hat{A}^{\prime} \hat{B}+\hat{A} \hat{B}^{\prime}\right) \operatorname{Re}\left\{\tilde{\varrho}_{k j}\left(\mathbf{r}, \mathbf{r}^{\prime}\right)\right\}\right|_{\mathbf{r}=\mathbf{r}^{\prime}}, \\
\frac{\delta \tilde{C}_{q}^{A, B}(\mathbf{r})}{\delta \kappa_{j k}} & =\left.\left(\hat{A}^{\prime} \hat{B}+\hat{A} \hat{B}^{\prime}\right) \operatorname{Im}\left\{\tilde{\varrho}_{j k}\left(\mathbf{r}, \mathbf{r}^{\prime}\right)\right\}\right|_{\mathbf{r}=\mathbf{r}^{\prime}}, \\
\frac{\delta \tilde{D}_{q}^{A, B \sigma}(\mathbf{r})}{\delta \kappa_{j k}} & =\left.\left(\hat{A}^{\prime} \hat{B}-\hat{A} \hat{B}^{\prime}\right) \operatorname{Re}\left\{\tilde{\varsigma}_{k j}\left(\mathbf{r}, \mathbf{r}^{\prime}\right)\right\}\right|_{\mathbf{r}=\mathbf{r}^{\prime}}, \\
\frac{\delta \tilde{C}_{q}^{A, B \sigma}(\mathbf{r})}{\delta \kappa_{j k}} & =\left.\left(\hat{A}^{\prime} \hat{B}-\hat{A} \hat{B}^{\prime}\right) \operatorname{Im}\left\{\tilde{\varsigma}_{k j}\left(\mathbf{r}, \mathbf{r}^{\prime}\right)\right\}\right|_{\mathbf{r}=\mathbf{r}^{\prime}},
\end{aligned}
$$

here the factor $1 / 2$ on the right-hand side of Eq. (161) originates from extending the summation over $j$ and $k$ in Eq. (75) to all combinations. Because of the skew symmetry of $\kappa_{i j}=-\kappa_{j i}$, the variation with respect to $\kappa_{i j}$ yields two contributions. Using the skew symmetry of $\tilde{\varrho}_{j k}\left(\mathbf{r}, \mathbf{r}^{\prime}\right)$, these can be combined again into a single term. This intermediate step is omitted in the other three relations. Analogously, one finds for their complex conjugates

$$
\begin{aligned}
& \frac{\delta \tilde{D}_{q}^{A, B *}(\mathbf{r})}{\delta \kappa_{j k}^{*}}=\left.\left(\hat{A}^{\prime} \hat{B}+\hat{A} \hat{B}^{\prime}\right) \operatorname{Re}\left\{\tilde{\varrho}_{k j}^{*}\left(\mathbf{r}, \mathbf{r}^{\prime}\right)\right\}\right|_{\mathbf{r}=\mathbf{r}^{\prime}}, \\
& \frac{\delta \tilde{C}_{q}^{A, B *}(\mathbf{r})}{\delta \kappa_{j k}^{*}}=\left.\left(\hat{A}^{\prime} \hat{B}+\hat{A} \hat{B}^{\prime}\right) \operatorname{Im}\left\{\tilde{\varrho}_{k j}^{*}\left(\mathbf{r}, \mathbf{r}^{\prime}\right)\right\}\right|_{\mathbf{r}=\mathbf{r}^{\prime}}, \\
& \frac{\delta \tilde{D}_{q}^{A, B \sigma *}(\mathbf{r})}{\delta \kappa_{j k}^{*}}=\left.\left(\hat{A}^{\prime} \hat{B}-\hat{A} \hat{B}^{\prime}\right) \operatorname{Re}\left\{\tilde{\varsigma}_{k j}^{*}\left(\mathbf{r}, \mathbf{r}^{\prime}\right)\right\}\right|_{\mathbf{r}=\mathbf{r}^{\prime}} \\
& \frac{\delta \tilde{C}_{q}^{A, B \sigma *}(\mathbf{r})}{\delta \kappa_{j k}^{*}}=\left.\left(\hat{A}^{\prime} \hat{B}-\hat{A} \hat{B}^{\prime}\right) \operatorname{Im}\left\{\tilde{\varsigma}_{k j}^{*}\left(\mathbf{r}, \mathbf{r}^{\prime}\right)\right\}\right|_{\mathbf{r}=\mathbf{r}^{\prime}}
\end{aligned}
$$

For sake of compact notation we omitted again the possible indices of the spatial cartesian tensor components of these objects.

For the pairing EDF (148) used for the calculations discussed in what follows, one obtains in new notation

$$
\begin{aligned}
\Delta_{j k}=\frac{\delta E_{\mathrm{pair}}}{\delta \kappa_{j k}^{*}} & =\int d^{3} r\left[\frac{\delta E_{\mathrm{pair}}}{\delta \tilde{D}_{q}^{1,1 *}(\mathbf{r})} \frac{\delta \tilde{D}_{q}^{1,1 *}(\mathbf{r})}{\delta \kappa_{j k}^{*}}+\frac{\delta E_{\mathrm{pair}}}{\delta \tilde{C}_{q}^{1,1 *}(\mathbf{r})} \frac{\delta \tilde{C}_{q}^{1,1 *}(\mathbf{r})}{\delta \kappa_{j k}^{*}}\right] \\
& =f_{j} f_{k} \int d^{3} r \frac{V_{q}}{2}\left[1-\frac{D_{0}^{1,1}(\mathbf{r})}{\rho_{c}}\right]\left[\tilde{D}_{q}^{1,1}(\mathbf{r}) \operatorname{Re}\left\{\tilde{\varrho}_{k j}^{*}(\mathbf{r}, \mathbf{r})\right\}+\tilde{C}_{q}^{1,1}(\mathbf{r}) \operatorname{Im}\left\{\tilde{\varrho}_{k j}^{*}(\mathbf{r}, \mathbf{r})\right\}\right], \\
& =f_{j} f_{k} \int d^{3} r\left[\tilde{F}_{q}^{1,1}(\mathbf{r}) \operatorname{Re}\left\{\tilde{\varrho}_{k j}^{*}(\mathbf{r}, \mathbf{r})\right\}+\tilde{G}_{q}^{1,1}(\mathbf{r}) \operatorname{Im}\left\{\tilde{\varrho}_{k j}^{*}(\mathbf{r}, \mathbf{r})\right\}\right], \\
\Delta_{j k}^{*}=\frac{\delta E_{\mathrm{pair}}}{\delta \kappa_{j k}} & =\int d^{3} r\left[\frac{\delta E_{\mathrm{pair}}}{\delta \tilde{D}_{q}^{1,1}(\mathbf{r})} \frac{\delta \tilde{D}_{q}^{1,1}(\mathbf{r})}{\delta \kappa_{j k}}+\frac{\delta E_{\mathrm{pair}}}{\delta \tilde{C}_{q}^{1,1}(\mathbf{r})} \frac{\delta \tilde{C}_{q}^{1,1}(\mathbf{r})}{\delta \kappa_{j k}}\right] \\
& =f_{j} f_{k} \int d^{3} r \frac{V_{q}}{2}\left[1-\frac{D_{0}^{1,1}(\mathbf{r})}{\rho_{c}}\right]\left[\tilde{D}_{q}^{1,1 *}(\mathbf{r}) \operatorname{Re}\left\{\tilde{\varrho}_{k j}(\mathbf{r}, \mathbf{r})\right\}+\tilde{C}_{q}^{1,1 *}(\mathbf{r}) \operatorname{Im}\left\{\tilde{\varrho}_{k j}(\mathbf{r}, \mathbf{r})\right],\right. \\
& =f_{j} f_{k} \int d^{3} r\left[\tilde{F}_{q}^{1,1, *}(\mathbf{r}) \operatorname{Re}\left\{\tilde{\varrho}_{k j}(\mathbf{r}, \mathbf{r})\right\}+\tilde{G}_{q}^{1,1, *}(\mathbf{r}) \operatorname{Im}\left\{\tilde{\varrho}_{k j}(\mathbf{r}, \mathbf{r})\right\}\right],
\end{aligned}
$$

where $f_{j}$ and $f_{k}$ are the cutoff factors of Eq. (149) and we have used that by definition $\delta \tilde{D}_{q}^{1,1}(\mathbf{r}) / \delta \kappa_{j k}^{*}=$ $\delta \tilde{C}_{q}^{1,1}(\mathbf{r}) / \delta \kappa_{j k}^{*}=\delta \tilde{D}_{q}^{1,1 *}(\mathbf{r}) / \delta \kappa_{j k}=\delta \tilde{C}_{q}^{1,1 *}(\mathbf{r}) / \delta \kappa_{j k}=$ 0 . From the definitions (75) and (76) follows directly that, in a gauge that leads to real $\kappa_{i j}=\kappa_{i j}^{*}$, one also automatically has real $\Delta_{j k}=\Delta_{j k}^{*}$, as all terms on the right-hand side of Eqs. (153) and (154) become integrals over real-valued functions.

\section{EXPLORATORY CALCULATIONS}

\section{A. Numerical choices}

We have extended the MOCCa code [41, 42] to allow for the solution of the self-consistent mean-field problem with N2LO EDFs of the form presented in the previous section. This code represents the self-consistent 
mean-field problem in coordinate space, utilizing the properties of Lagrange meshes [62, 63], for which the derivatives of a function are constructed from the values of said function on all mesh points. Together with a rectangular quadrature rule, the Lagrange-mesh derivatives implicitly define an underlying basis of plane waves in a box 64]. The Lagrange-mesh technique has the advantages over simpler finite-difference expressions for derivatives that integration by parts is exact up to machine precision, and that higher-order derivative matrices can be calculated as products of matrices of first-order derivatives. For N2LO EDFs, these two features turn out to be even more important for the suppression of numerical noise brought by the higher number of repeated derivatives needed when calculating the local densities and when applying the single-particleHamiltonian for the Skyrme N2LO functional than for the standard NLO functional.

Using this representation, one obtains the total energy of nuclear configurations with an accuracy that is essentially independent of their deformation [64]. In addition to the stringent numerical tests mentioned in Sec. IVB above, MOCCa was also benchmarked in detail against the spherical code used in Ref. [36]. For all calculations reported here, we have set the mesh spacing $d x$ to $0.8 \mathrm{fm}$ while adapting the box size to the nucleus under consideration, as in Ref. 64].

The only presently available Skyrme parametrization for finite nuclei that includes N2LO terms is SN2LO1 36]. For the purposes of comparison, we also report on calculations with two NLO parametrizations. For all nuclei considered below, we include results for the SLy5* parametrization [40]. This parametrization serves as a good point of comparison, as the fit protocol constructed for its adjustment was later also adopted for SN2LO1 with only minimal changes. When discussing very heavy nuclei, we also include results obtained with the NLO SLy5s1 parametrization that was adjusted with an extension of said fit protocol that incorporated an additional constraint on surface tension [65]. We include SLy5s1 because of its superior performance for deformation properties of heavier nuclei that results from its more realistic surface tension [66].

All three of these parametrizations were adjusted for doubly-magic nuclei for which pairing correlations vanish at the mean-field level. In order to include the effect of pairing correlations, we have added a pairing term (148) to the EDF that corresponds to a density-dependent zerorange pairing interaction, supplemented with a smooth cutoff both above and below the Fermi energy, as originally proposed in Ref. [59, 60]. As the effective mass of all three parametrizations considered is almost identical to that of SLy4 for which this pairing EDF has been adjusted, we decided to use the same parameters $V_{n}=V_{p}=-1250 \mathrm{MeV} \mathrm{fm}^{3}$ and $\rho_{c}=0.16 \mathrm{fm}^{-3}$ of the pairing EDF and the same cutoff parameters as adjusted for SLy4 in Refs. 59, 60] in all calculations. This has the additional advantage of making the comparison between the three Skyrme EDFs more straightforward. The resulting HFB equations were solved using the two-basis method [46, 61]. We have employed the Lipkin-Nogami procedure to avoid the collapse of pairing correlations [61].

If one wants to link the Skyrme EDF to an underlying pseudopotential, the coupling coefficients of the time-odd terms are completely determined when one has fixed the coupling coefficients of the time-even terms. For many Skyrme NLO parametrizations, however, making such a choice is impossible in practice, as they exhibit finitesize instabilities with respect to spin polarization which show up in situations when time-reversal symmetry is broken. Examples are calculations with brokenpair blocked quasiparticles 67], self-consistent cranked calculations [11], QRPA calculations 43], or timedependent calculations [68]. When working with NLO parametrizations that exhibit such instability, one and/or the other of the coupling constants of the $A_{t, \mathrm{o}}^{(2,1)} \mathbf{D}_{t}^{1, \sigma}(\mathbf{r})$. $\left[\Delta \mathbf{D}_{t}^{1, \sigma}(\mathbf{r})\right]$ term in Eq. (119) (and/or the coupling constants of a similar term proportional to $\left[\boldsymbol{\nabla} \cdot \mathbf{D}_{t}^{1, \sigma}(\mathbf{r})\right]^{2}$ that only contributes when including explicit tensor forces [11] not considered here), has to be set to smaller value, which is typically chosen to be zero.

The empirical criteria developed in Ref. [69] for isospin instabilities have been sufficient to guard also against such unphysical spin instabilities in the cases of SLy5* 40] and SLy5s1 [46]. For SN2LO1, the same techniques were used with the same empirical protocol to safeguard the adjustment of the parametrization, and we aim to validate their effectiveness for the case of N2LO parametrizations. For this reason, we have kept all timeodd terms in the particle-hole channel as generated by the underlying pseudopotential as considered in Ref. 36, 40, 65] for all three parametrizations.

As demonstrated in Ref. [46], the presence of higher-order derivatives in the functional renders the convergence of the iterative solution of the self-consistent problem more difficult to control numerically. We have employed the heavy-ball and preconditioning algorithms of that reference to reliably and quickly converge the calculations.

We will present results as a function of the dimensionless (mass) quadrupole multipole moment, defined as

$$
\beta_{20}=\frac{4 \pi}{3 R_{0}^{2} A}\left\langle r^{2} Y_{20}\right\rangle
$$

where $Y_{20}$ is a spherical harmonic and $R_{0}=1.2 A^{1 / 3}$ fm. Positive (negative) values of $\beta_{20}$ indicate that the nuclear configuration exhibits a prolate (oblate) shape. In order to compare to experimental data obtained from E2 transitions, we also define the dimensionless charge deformation

$$
\beta_{20, p}=\frac{4 \pi}{3 R_{0}^{2} Z}\left\langle r_{p}^{2} Y_{20, p}\right\rangle,
$$



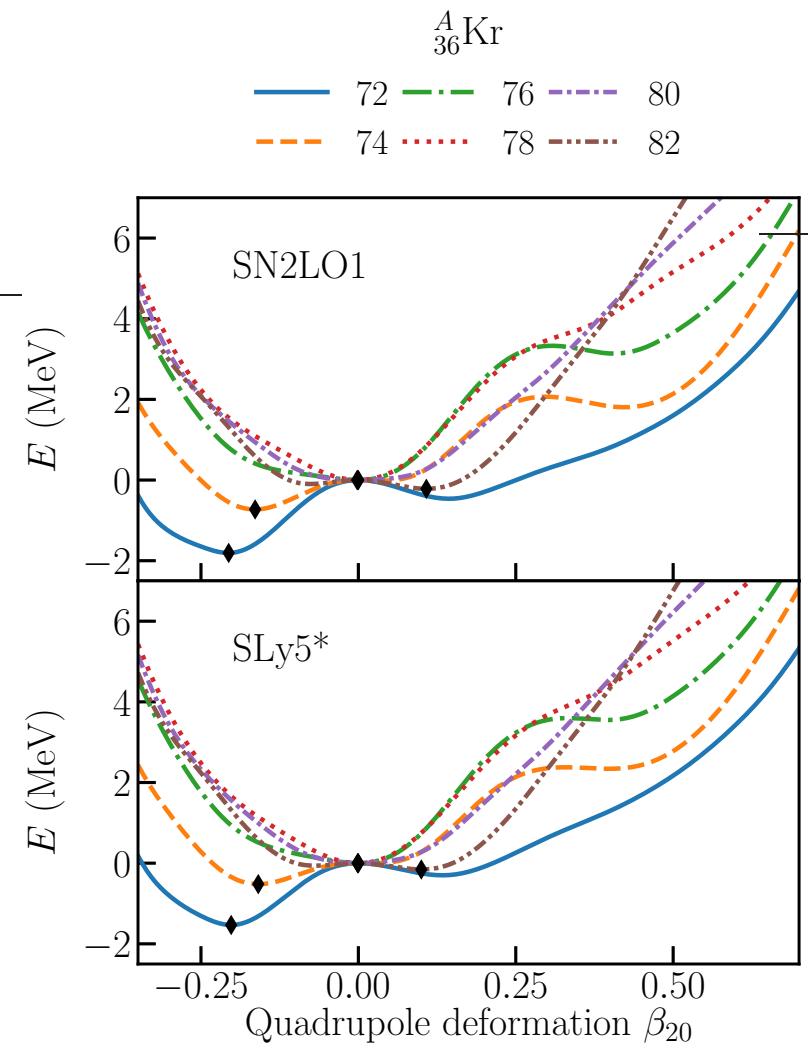

FIG. 1. Deformation energy curve for the lighter krypton isotopes ${ }^{72-82} \mathrm{Kr}$ as a function of the quadrupole moment $\beta_{20}$, normalized to the spherical configurations. Top: results for SN2LO1, bottom, results for SLy5*. Black diamond indicate the global minimum for each isotope.

which usually takes values that differ from those of $\beta_{20}$ by less than a few percent.

Finally, we will also compare to experimental data on isotopic shifts. For a nucleus with $N$ neutrons, the isotopic shift is defined as the difference between its mean-squared (ms) charge radius and the ms radius of a reference isotope of the same element with $N_{0}$ neutrons as

$$
\delta\left\langle r_{p}^{2}\right\rangle^{N, N_{0}}=\left\langle r_{p}^{2}\right\rangle^{N}-\left\langle r_{p}^{2}\right\rangle^{N_{0}}
$$

\section{B. Shape coexistence in the Krypton isotopes}

We first consider the deformation energy curves of even-even krypton $(Z=34)$ isotopes, spanning all isotopes from $N=36$ up to $N=58$. Figures [1 and 2 show the energy curves of ${ }^{72-82} \mathrm{Kr}$ and of ${ }^{84-94} \mathrm{Kr}$ as calculated with the SN2LO1 parametrization (top panels) and calculated with the SLy5* parametrization (bottom panels). All curves have been normalized to the energy of the spherical configuration.

The two parametrizations produce strikingly similar potential energy curves. For both parametrizations the
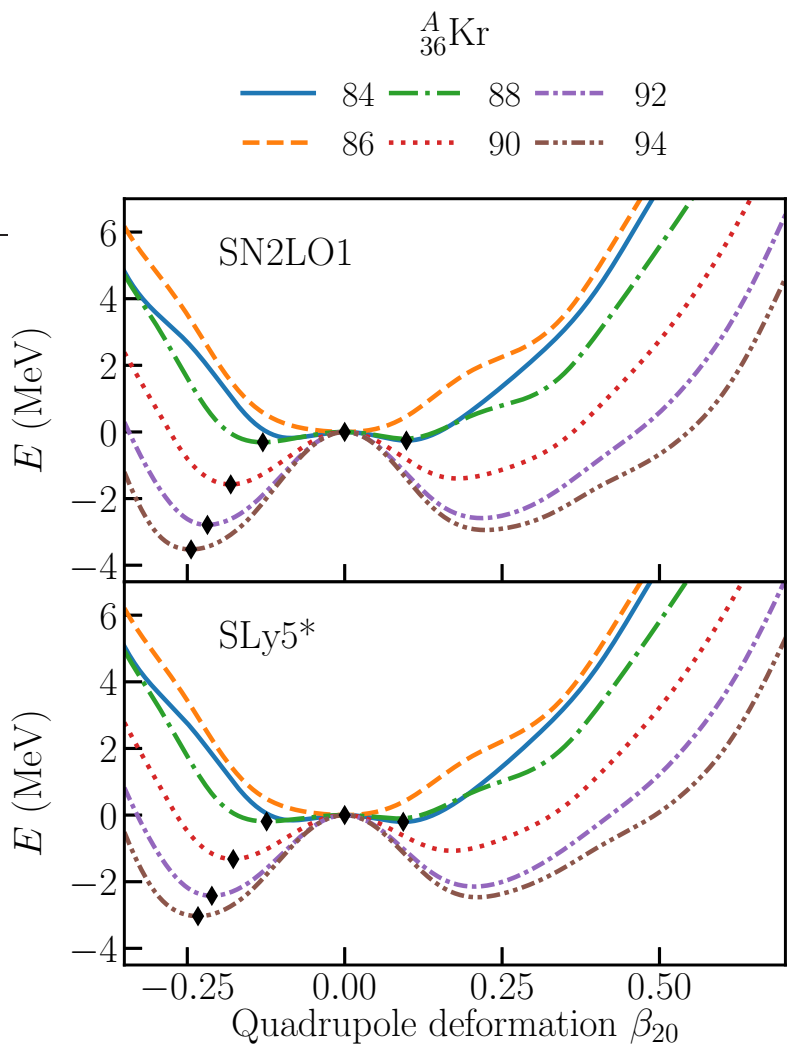

FIG. 2. Same as Fig. 1 but for the heavier isotopes ${ }^{84-94} \mathrm{Kr}$.

neutron-deficient $A=72$ and 74 isotopes exhibit a pronounced oblate minimum, which evolves to a spherical symmetric minimum for $A=76,78,80$, and also 86. ${ }^{82} \mathrm{Kr}$ exhibits a very shallow prolate minimum, as does ${ }^{84} \mathrm{Kr}$. From ${ }^{88} \mathrm{Kr}$ onwards, the isotopes develop a pronounced oblate minimum again. While minute differences between the results for both parametrizations exist, this general evolution is identical. This does not mean that the N2LO terms of SN2LO1 do not contribute to the energy: the contribution of $\mathcal{E}_{\mathrm{e}}^{(4)}(\mathbf{r})$ to the total energy of the spherical configuration of ${ }^{76} \mathrm{Kr}$ integrates to roughly $-38 \mathrm{MeV}$ which is not negligible, but remains a small fraction of the total binding energy of about 800 $\mathrm{MeV}$. The contribution of the N2LO terms varies on the level of a few $\mathrm{MeV}$ as a function of deformation, but does not produce any meaningful shifts of the overall topography, as evidenced by Figs. 11and 2, For the other nuclei discussed in what follows, the N2LO terms behave similarly.

The neutron-deficient ${ }^{72-78} \mathrm{Kr}$ isotopes are a prime example of shape coexistence. Detailed data from Coulomb excitation experiments for these nuclei can be interpreted in terms of a highly-deformed prolate and a lesser-deformed oblate (or possibly non-axial) structure that quickly change their relative position in the energy spectrum with neutron number. The situation is simplest for ${ }^{76} \mathrm{Kr}$ and ${ }^{78} \mathrm{Kr}$, which have a strongly deformed 
prolate ground state [70, 71] with $\beta_{2} \simeq 0.4$ that is the band-head of a rotational band built from states with similar deformations. Going from ${ }^{78} \mathrm{Kr}$ to ${ }^{76} \mathrm{Kr}$, the excitation energy of the $0_{2}^{+}$state goes down from $1.017 \mathrm{MeV}$ to $0.760 \mathrm{MeV}$, and even further down to $0.509 \mathrm{MeV}$ in ${ }^{74} \mathrm{Kr}$. While the overall band structure of ${ }^{74} \mathrm{Kr}$ is similar to the one of the heavier isotopes, the measured transition moments indicate that the two coexisting structures are strongly mixed in the low-lying states 71]. For the even lighter $N=Z$ isotope ${ }^{72} \mathrm{Kr}$, a recent measurement finds that the ground-state has an oblate shape, while the yrast states of higher spin remain prolate [72]. Beginning with ${ }^{80} \mathrm{Kr}$, the low-lying part of the spectrum of the heavier isotopes around $N=50$ can rather be interpreted in terms of anharmonic vibrations 73 .

This scenario is not reproduced by either of the Skyrme parametrizations. Both of them predict either oblatedeformed or near-spherical shapes for all $\mathrm{Kr}$ isotopes in Figs. 1 and 2. They both also produce a local minimum at large prolate deformation for ${ }^{74-76} \mathrm{Kr}$, but these configurations are still several $\mathrm{MeV}$ above the calculated oblate minimum. This is a problem that SLy5* and SN2LO1 share with many other modern parametrizations of the Skyrme EDF 66, 74], while an early one like SIII correctly gives a prolate ground state for ${ }^{74-76} \mathrm{Kr}$ [75]. Similar problems are also found for the ground states of $\mathrm{Zr}$ isotopes [10, 66]. It has been argued in Ref. [74] that this finding might be caused by incorrect relative distances between single-particle levels at spherical shape in this mass region. In any event, the differences between SN2LO1 and SLy5* are much smaller than what is typically found when comparing with results from other NLO Skyrme parametrizations for nuclei in this mass region [10, 66], which probably can be attributed to the similarity of their fit protocol.

On the other hand, using a Gogny interaction the shape transition and the overall structure of lowlying excited states in the neutron-deficient Kr isotopes has been successfully modeled in beyond-mean-field calculations when exactly or approximatively projecting on angular momentum and mixing states in the entire $\beta-\gamma$ plane [71, 72, 76, 77], which might hint at the insufficiency of pure mean-field calculations to describe shape coexistence phenomena in this mass region.

\section{Deformation properties of the neodynium isotopes}

As a second example we consider the chain of $\mathrm{Nd}$ $(Z=60)$ isotopes, which present a medium-heavy set of nuclei that offer a large range of deformations that evolve from strongly deformed shapes of the neutrondeficient isotopes over spherical ones near $N=82$ to again strongly deformed neutron-rich isotopes. Figure 3 confronts calculated isotopic shifts of charge radii obtained with the SN2LO1 and SLy5* parametrizations

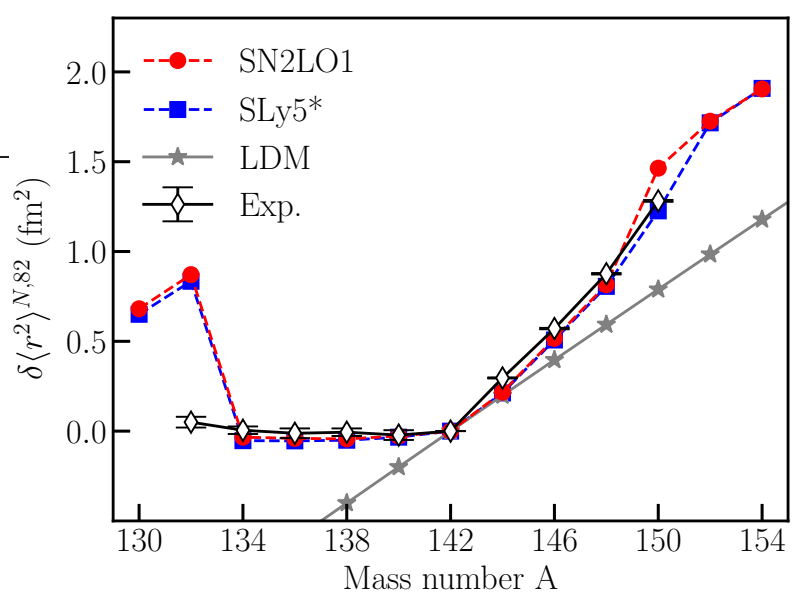

FIG. 3. Isotopic shifts $\delta\left\langle r^{2}\right\rangle^{N, 82}$ of mean-square charge radii for the Nd isotope chain calculated with SLy5* and SN2LO1. Experimental values were taken from Ref. [78]. The evolution of charge radii when assuming the global $A^{2 / 3}$ scaling of the spherical liquid-drop model is also given for comparison.

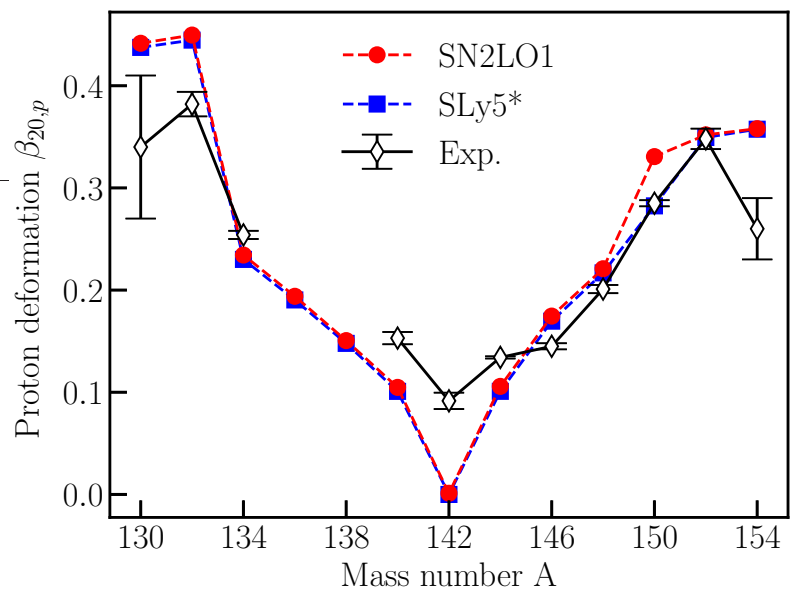

FIG. 4. Deformation $\beta_{20, p}$ of the proton distribution in the Nd isotope chain calculated with SLy5* and SN2LO1, compared to values of the transition charge deformation deduced from experimental $B\left(E 2,0_{1}^{+} \rightarrow 2_{1}^{+}\right)$values [79] under the assumption that the nuclei are rigid rotors.

with the available data, whereas Fig. 4 compares the deformation $\beta_{20, p}$ of protons in the calculated ground states of these nuclei with the experimental transition charge deformation as deduced from $B\left(E 2,0_{1}^{+} \rightarrow 2_{1}^{+}\right)$ values [79].

The isotopic shifts of the $\mathrm{Nd}$ isotopes exhibit a clear kink at $A=142$, such that their overall growth does not follow the global scaling of ms charge radii with $A^{2 / 3}$. The latter is indicated on the figure by plotting the ms radius of a spherical liquid-drop $r^{2}=(3 / 5) r_{0}^{2} A^{2 / 3}$, where the surface radius constant $r_{0}$ is determined through $r_{0}^{3}=3 /\left(4 \pi \rho_{\text {sat }}\right)$ from the average of the very similar saturation densities of SLy5* and SN2LO1. The 
deviations from this smooth trend can be attributed to the gradual onset of deformation on both sides of $N=82$ shell closure 80]. From the structure of their excitation spectrum [81], nuclei in the direct vicinity of ${ }^{142} \mathrm{Nd}$ should be interpreted in terms of near-spherical soft anharmonic vibrators, such that their $B\left(E 2,0_{1}^{+} \rightarrow 2_{1}^{+}\right)$values cannot be reliably linked to an intrinsic deformation through that assumption of a rigid rotor model. In fact, rotational bands built on the ground state have been observed for $A \leq 136$ and $A \geq 146$, but their level spacing approaches the one of a rigid rotor only for $A \lesssim 136$ and $A \gtrsim 150$ [81. The ms charge radii from the mean-field ground states nevertheless follow closely the trend of the experimental ones.

The calculations overestimate the deformation and charge radii for the very neutron-deficient isotopes, but otherwise reproduce the experimental trend rather well across the entire chain.

As for the $\mathrm{Kr}$ isotopes, the calculated ground-state deformations of all $\mathrm{Nd}$ isotopes are very similar for the two parametrizations, with the SN2LO1 ones being marginally larger for all nuclei. Consequently, the calculated isotopic shifts are also very similar, with the SN2LO1 values always being slightly higher. The largest difference between parametrizations is found for ${ }^{150} \mathrm{Nd}$ : the deformation energy surface of this nucleus is very flat around $\beta_{20} \simeq 0.3$, such that a small difference in any one aspect of a parametrization can induce a rather large change of deformation for this nucleus.

\section{The fission barrier of ${ }^{240} \mathbf{P u}$}

Figure 5 shows the static fission barrier of ${ }^{240} \mathrm{Pu}$, calculated with the three parametrizations SN2LO1, SLy5* and SN2LO1, as a function of the dimensionless mass quadrupole moment $\beta_{20}$ as defined in Eq. (171). This nucleus provides one of the standard test benches for models of fission, two of the reasons being the availability of experimental data for several of the characteristic energies of its fission barrier, as well as the possibility to obtain a continuous fission path by making calculations with a single constraint. Moving from small to large deformations, the lowest mean-field configuration exhibits shapes with different intrinsic symmetries. Shapes are axial around the local minima, but both around the inner and outer saddle points the nucleus takes non-axial shapes. Up to deformations slightly beyond the fission isomer, shapes remain reflection symmetric, whereas beyond the configurations become increasingly reflection-asymmetric. Along the respective fission paths, the values of all multipole deformations up to at least $\ell=8$ are near-identical for all three parametrizations. In particular, around the outer saddle point, the favored nuclear shapes combine non-axiality and reflection asymmetry for all three parametrizations, a feature that was already reported on for SLy5s1 in Ref. [66], the UNEDF1 parametrization of the Skyrme

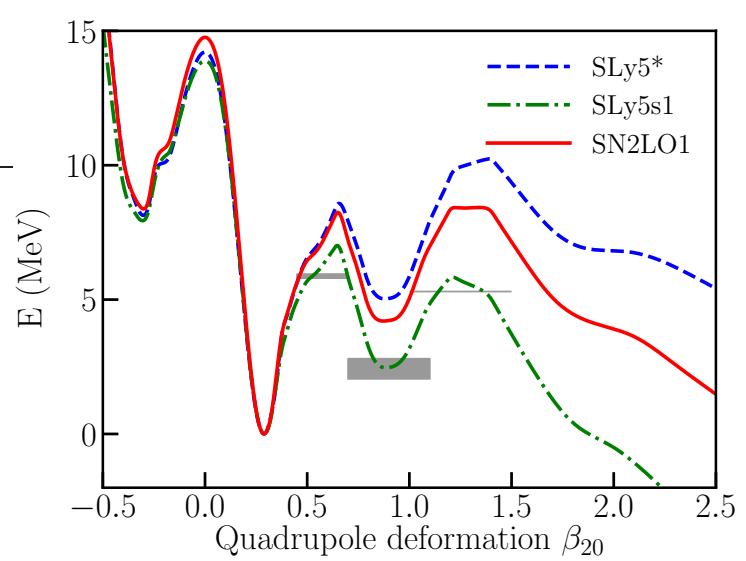

FIG. 5. Static fission path of ${ }^{240} \mathrm{Pu}$ as a function of quadrupole deformation $\beta_{20}$, as calculated with the SLy5s1, SLy5* and SN2LO1 parametrizations. The energy curves are normalized to their respective prolate minimum. Along the path, nuclear shapes explore non-axial and/or reflection asymmetric deformations (see text). The horizontal grey bars indicate experimental values for the height of the inner and outer barriers as well as the excitation energy of the fission isomer. taken from the same sources as in Ref. [66]

EDF at NLO in Ref. 82, and for relativistic EDF approaches in Ref. [83].

At small deformation, the overall profile of the barrier is very similar for all three parametrizations. Only for $\beta_{20} \geq 0.5$ do we observe deviations larger than 1 MeV. Between SLy5s1 and SLy5*, this difference can be explained through the variation in surface tension of these parametrizations: with $a_{\text {surf }}^{\mathrm{HF}}=18.61 \mathrm{MeV}$, the surface energy coefficient of $\mathrm{SLy}^{*}$ is much larger than the one of SLy5s1 that takes the value of $a_{\text {surf }}^{\mathrm{HF}}=$ $17.55 \mathrm{MeV}$ [65], resulting in a larger loss in binding energy with increasing deformation. The correlation between the surface tension and the height of the fission barrier of ${ }^{240} \mathrm{Pu}$ has been systematically studied in Refs. 65, 66], concluding that parametrizations with low surface tension like SLy5s1 in general provide a better description of the experimental data on fission barriers and deformation properties of heavy nuclei. Unfortunately, there is no tool available yet to calculate the surface energy coefficient of N2LO parametrizations like SN2LO1. The observation that the deformation energy curve obtained with SN2LO1 falls systematically between those for SLy5s1 and SLy5* suggests that also its surface tension is in between the ones of these parametrizations. With that, SN2LO1 brings an improvement compared to SLy5*, but a further finetuning of its surface properties along the lines of Ref. 65. will be needed to arrive at realistic predictions for fission barriers. 


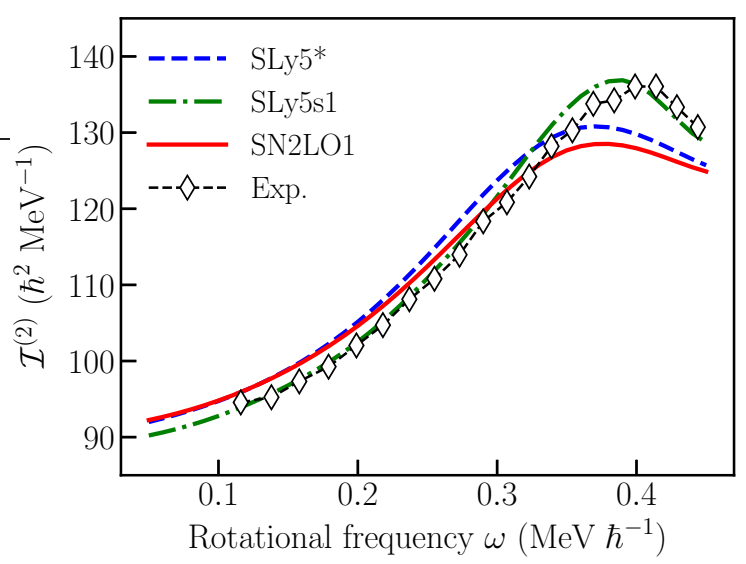

FIG. 6. Dynamical moment of inertia $\mathcal{I}^{(2)}$ of the superdeformed band in ${ }^{194} \mathrm{Hg}$ as a function of the cranking frequency $\omega$ for the SLy5s1, SLy5* and the SN2LO1 parametrizations. As before, results for NLO (N2LO) parametrizations are plotted with dashed (full) lines. The empty diamond symbols are experimental results for the yrast SD-1 band taken from Ref. [84].

\section{E. Superdeformed rotational band in ${ }^{194} \mathbf{H g}$}

As a final example for yet another type of symmetrybreaking in mean-field calculations, we studied the properties of the yrast superdeformed rotational band of ${ }^{194} \mathrm{Hg}$, which is another often-used test bench for properties of the Skyrme EDF, in particular its time-odd part [11, 66]. We will focus on the dynamical moment of inertia $\mathcal{I}^{(2)}$, which is very sensitive to changes of the nuclear mean field in response to the nucleus' rotation. Modelling this band as the semiclassical rotation of a deformed nuclear shape, this quantity is defined as

$$
\mathcal{I}^{(2)} \equiv\left(\frac{d^{2} E}{d J^{2}}\right)^{-1}
$$

where $E$ is the total binding energy energy and $J$ is the size of the (classical) angular momentum. The moment of inertia $\mathcal{I}^{(2)}$ is usually studied as a function of the rotational frequency

$$
\omega \equiv \frac{d E}{d J}
$$

To connect these quantities to experimentally accessible information, it is customary to calculate Eqs. (174) and (175) through finite differences of in-band $\gamma$-ray transition energies [84].

In a self-consistent mean-field model, the moment of inertia and rotational frequency can be accessed through cranked calculations, where, instead of the energy $E$, one minimizes the Routhian $R$

$$
R(\omega)=E-\omega_{\text {crank }}\left\langle\hat{J}_{z}\right\rangle
$$

as a function of the frequency $\omega_{\text {crank }}$. In this procedure, the cranking frequency serves as the Lagrange parameter for a constraint on the angular momentum along the z-axis, which breaks time-reversal invariance. In such a calculation, all time-odd densities can take non-zero values, and the calculation reported on here is, to the best of our knowledge, the first that probes the timeodd terms of a N2LO parametrization in a finite nucleus. Shapes have been limited to triaxial ones as done in earlier calculations of this band [11, 59, 61, 66].

Figure 6 confronts calculated values for the dynamical moment of inertia along the super-deformed band of ${ }^{194} \mathrm{Hg}$ with experimental data on the band labelled "SD1" from Ref. [84]. In order to make the comparison, we have identified $\omega_{\text {crank }}$ with the rotational frequency $\omega$ as deduced from observed $\gamma$-ray energies. Calculations with SN2LO1 and SLy5* produce almost identical curves, while the SLy5s1 moments of inertia are slightly lower at low spin, but then cross over and become slightly higher at high spin. Overall, all three parametrizations provide a good descriptions of the experimental data, with a slight preference for SLy5s1. The differences between them, however, are in fact on the same scale or even smaller than what is typically found when comparing different Skyrme NLO parameter sets [11, 66], or different pairing models for the same parameter set 59], or when slightly varying pairing strengths [11, 59] or specific coupling constants of NLO time-odd terms that are under-constrained by present fit protocols [11].

A more technical point implied by Figure 6 is that the SN2LO1 parametrization is stable with respect to spurious finite-size instabilities in the spin channel even when all time-odd terms are taken into account that are generated by the underlying two-body pseudopotential. These calculations validate a posteriori also for N2LO EDFs the stability criteria proposed in Ref. [69] that were used during the parameter adjustment of SLy5s1 65] and SN2LO1 [36]. The earlier SLy5* [40] was adjusted along the same lines, which assured for the first time the stability of Skyrme EDFs against finite-size spin instabilities that are exhibited by many parameter sets of the Skyrme NLO EDF when using coupling constants $A_{t, \mathrm{o}}^{(2,1)}$ of the $\mathbf{D}_{t}^{1, \sigma}(\mathbf{r}) \cdot\left[\Delta \mathbf{D}_{t}^{1, \sigma}(\mathbf{r})\right]$ terms in the NLO EDF (119) as obtained from a generating two-body pseudopotential [43, 69].

\section{CONCLUSIONS, SUMMARY AND OUTLOOK}

We have presented an exploratory study of deformed nuclei using the N2LO Skyrme EDF proposed in Ref. [36], addressing the optimal formal and numerical representation of such extended Skyrme functionals in terms of local densities, and analyzing the performance of the SN2LO1 parameter set of Ref. [36].

Our main observations and conclusions concerning the formal representation of the Skyrme EDF at N2LO are

(i) Going from NLO to N2LO requires the introduction of additional local 
densities 21, 24, 34, 36 that either contain additional gradients, or that have a higherrank cartesian tensor structure. For the N2LO functional generated from a locally gaugeinvariant central two-body pseudopotential with four gradients considered here, four new normal densities as well as two generalizations of previously defined densities to higher tensor rank are needed. The notation that was employed in the first exploratory studies of such EDF in Refs. 34, 36] is not very transparent: it leads to an inflation in the number of symbols, none of which provides an indication of the operator structure of a given density and thereby limits the extensibility of this strategy to arbitrary order in gradients.

(ii) Several of the densities introduced in Refs. [34, 36] to define the N2LO functional turn out to be complex spatial functions and therefore do not exhibit definite behavior under time-reversal.

(iii) As a solution to the problems and ambiguities concerning notation, we propose a new scheme for writing local densities and the corresponding mean fields. This notation indicates in a natural way the operator structure of densities and potentials at any order in gradients. Also, it produces by construction only densities that are real functions with definite behavior under time-reversal and thereby naturally separates the parts of the N2LO functional that lead to time-even and time-odd mean fields.

(iv) It turns out that some components of the higherorder densities introduced in Refs. 34, 36 to define the N2LO functional can be expressed through gradients acting on lower-order densities. As the latter are much simpler to handle formally and numerically, the use of such reducible densities should be avoided. The new notation introduced here helps to identify densities that are nonreducible in that sense.

(v) The definition of the Skyrme EDF in terms of local densities is not unique. Beginning at NLO, a given EDF can be expressed through several different, but equivalent sets of densities that differ by a recoupling of gradients. To limit the number of densities and their associated potentials to be calculated and stored, the use of redundant densities, that is densities that can be expressed as a linear combination of other densities and their gradients, should clearly be avoided.

(vi) Profiting from the new notation and the freedom to recouple gradients, we propose a set of rules that guide choices for the construction of a set of non-redundant and non-reducible densities that are advantageous in terms of the computational cost of the floating-point operations necessary to construct a given local density and to apply the corresponding term in the single-particle Hamiltonian on a single-particle state, and also in terms of the memory size required to store the derivatives of single-particle states needed along the way. The set of densities proposed in Sec. IIID offers in our opinion the most straightforward and efficient way to represent the local, central N2LO functional of Ref. 36].

We believe that the further exploration of degrees of freedom associated with higher-order gradients can benefit greatly from a more systematic notation like the one proposed here and its consequent categorization of densities. Not only does it allow to more readily identify reducibility and redundancies in any given set of local densities, it opens to the way to automated implementations of such complicated functionals. Already for the N2LO functional form of Ref. 34, 36] that is limited to central and locally gauge-invariant terms, a manual implementation is timeconsuming and error-prone, especially in the context of symmetry-breaking codes where every component of vector and tensor densities exhibits different symmetry properties. The future implementation of other higherorder terms such as the N2LO tensor pseudopotential and N3LO terms in general [30 33] in a deformed nuclear structure code will be even more laborious in this respect.

In that context, we recall that also the numerical treatment of N2LO terms in a self-consistent meanfield solver is not entirely straightforward because of the contributions to the single-particle Hamiltonian that are of third and fourth order in gradients. Such terms cannot be treated with some of the widely-used algorithms to represent and solve the self-consistent mean-field equations 36. It turns out, however, that the N2LO terms can be very precisely handled in a coordinatespace representation based on a Lagrange-mesh, which consistently treats derivatives of all orders [46], without any need for new developments. This is the technique we use here. While methods to directly integrate up a differential equation might face problems in the context of N2LO EDFs, iterative schemes in the spirit of the gradient-descent method to diagonalize the singleparticle Hamiltonian can be applied without fundamental technical difficulties at N2LO. The convergence of such methods, however, can become an issue, but algorithms exist that offer comparable efficiency for NLO and N2LO functionals [46]. These are the techniques we use here.

We have also reported on the first symmetrybreaking self-consistent calculations with existing parametrizations of the N2LO functional beyond the convergence tests presented in Ref. [46]:

(i) The examples cover typical applications for heavy nuclei: ground-state deformation and its impact on charge radii, rotational bands, and fission barriers. For their description, we had to consider a multitude of broken symmetries: breaking of 
rotational symmetry considering both axial and non-axial configurations, breaking of reflection symmetry, and breaking of time-reversal symmetry. All of these are frequently considered and explored in calculations of properties of finite nuclei.

(ii) The example of the superdeformed band of ${ }^{194} \mathrm{Hg}$ indicates that SN2LO1 is stable against finite-size spin-instabilities, such that time-reversal breaking calculations can be safely performed with this parametrization.

(iii) The results presented here, and others not mentioned, indicate that the SN2LO1 parametrization shares both the successes and failures of the NLO parametrizations: SN2LO1 describes the properties of deformed finite nuclei about as well as the NLO parameter set SLy5* that was adjusted with the same fit protocol. Differences between these two parameter sets are much smaller than what is typically found when comparing NLO parametrizations obtained with different fit protocols.

(iv) While a careful covariance analysis has shown that the coupling constants of the N2LO terms considered for SN2LO1 are from a statistical point of view significantly different from zero [37], they remain rather small and do neither significantly nor systematically improve the agreement with experiment compared to parametrizations of an NLO EDF adjusted with the same fit protocol, at least for the set of results and observables studied here.

Altogether, our results demonstrate that the N2LO functional of Ref. 34 37] can be reliably used to describe the structure of complex nuclei. There is, however, a clear need to better constrain the EDF during the parameter adjustment with observables that are particularly sensitive to the additional degrees of freedom offered by the new N2LO terms and can discriminate them from the terms in the standard NLO functional. Work in that direction is underway, as is the further extension of the Skyrme EDF through N2LO tensor terms as well as N3LO terms 30 33].

\section{ACKNOWLEDGMENTS}

We wish to thank Pierre Becker, Karim Bennaceur, Dany Davesne and Jacques Meyer for many stimulating discussions that helped to shape the material presented in this paper, and Paul Proust for many fruitful discussions on its efficient presentation. The work of W. R. was in part supported by the U.S. DOE grant No. DE-SC0019521, and in part by the FNRS (Belgium). The work of M.B. was supported by the french Agence Nationale de la Recherche under grant No. 19-CE31-0015-01 (NEWFUN). The computations were performed using HPC resources from the Consortium des Équipements de Calcul Intensif (CÉCI), funded by the Fonds de la Recherche Scientifique de Belgique (F.R.S.FNRS) under Grant No. 2.5020.11, the computing center of the IN2P3/CNRS as well as the computing resources of Yale University.

\section{Appendix A: Transforming the functional from the original of Ref. [36]}

In this appendix, we show explicitly how to transform the N2LO energy density of Ref. [36], Eq. (5), into the form we propose, Eqs. (117) and (120). We start by rewriting Eq. (5) in the new notation, employing the dictionary of subsection IIIC and separating the energy density into time-even and time-odd parts $\mathcal{E}_{t}^{(4)}(\mathbf{r})=$ $\mathcal{E}_{t, \mathrm{e}}^{(4)}(\mathbf{r})+\mathcal{E}_{t, \mathrm{o}}^{(4)}(\mathbf{r})$,

$$
\begin{aligned}
\mathcal{E}_{t, \mathrm{e}}^{(4)}(\mathbf{r})= & +C_{t}^{(4) \Delta \rho}\left[\Delta D_{t}^{1,1}(\mathbf{r})\right]^{2} \\
& +C_{t}^{(4) M \rho}\left\{D_{t}^{1,1}(\mathbf{r}) D_{t}^{\Delta, \Delta}(\mathbf{r})+\left[D_{t}^{(\nabla, \nabla)}(\mathbf{r})\right]^{2}+2 \sum_{\mu \nu} D_{t, \mu \nu}^{\nabla, \nabla}(\mathbf{r}) D_{t, \mu \nu}^{\nabla, \nabla}(\mathbf{r})-2 \sum_{\mu \nu} D_{t, \mu \nu}^{\nabla, \nabla}(\mathbf{r})\left[\nabla_{\mu} \nabla_{\nu} D_{t}^{1,1}(\mathbf{r})\right]\right\} \\
& +C_{t}^{(4) M s}\left\{-2 \sum_{\mu \nu \kappa} C_{t, \mu \nu \kappa}^{\nabla, \nabla \sigma}(\mathbf{r}) C_{t, \mu \nu \kappa}^{\nabla, \nabla \sigma}(\mathbf{r})-\sum_{\nu}\left[\sum_{\mu} \nabla_{\mu} C_{t, \mu \nu}^{1, \nabla \sigma}(\mathbf{r})\right]^{2}-4 \sum_{\mu \nu} C_{t, \mu \nu}^{1, \nabla \sigma}(\mathbf{r}) C_{t, \mu \nu}^{(\nabla, \nabla) \nabla \sigma}(\mathbf{r})\right\}, \\
\mathcal{E}_{t, \mathrm{o}}^{(4)}(\mathbf{r})= & +C_{t}^{(4) \Delta s}\left[\Delta \mathbf{C}_{t}^{1, \sigma}(\mathbf{r})\right]^{2} \\
& +C_{t}^{(4) M \rho}\left\{-2 \sum_{\mu \nu} C_{t, \mu \nu}^{\nabla, \nabla}(\mathbf{r}) C_{t, \mu \nu}^{\nabla, \nabla}(\mathbf{r})-\left[\nabla \cdot \mathbf{C}_{t}^{1, \nabla}(\mathbf{r})\right]^{2}-4 \mathbf{C}_{t}^{1, \nabla}(\mathbf{r}) \cdot \mathbf{C}_{t}^{(\nabla, \nabla) \nabla}(\mathbf{r})\right\}
\end{aligned}
$$




$$
\begin{aligned}
+C_{t}^{(4) M s} & \left\{\mathbf{D}_{t}^{1, \sigma}(\mathbf{r}) \cdot \mathbf{D}_{t}^{\Delta, \Delta \sigma}(\mathbf{r})+\left[\mathbf{D}_{t}^{(\nabla, \nabla) \sigma}(\mathbf{r})\right]^{2}+2 \sum_{\mu \nu \kappa} D_{t, \mu \nu \kappa}^{\nabla, \nabla \sigma}(\mathbf{r}) D_{t, \mu \nu \kappa}^{\nabla, \nabla \sigma}(\mathbf{r})\right. \\
& \left.-2 \sum_{\mu \nu \kappa} D_{t, \mu \nu \kappa}^{\nabla, \nabla \sigma}(\mathbf{r})\left[\nabla_{\mu} \nabla_{\nu} D_{t, \kappa}^{1, \sigma}(\mathbf{r})\right]\right\}
\end{aligned}
$$

We draw the readers attention to the minus signs in front of the terms bilinear in the reducible currents $C_{t, \mu \nu}^{\nabla, \nabla}(\mathbf{r})$ and $C_{t, \mu \nu \kappa}^{\nabla, \nabla \sigma}(\mathbf{r})$, which result from the square of the imaginary unit i. We also remark that the term $\sum_{\mu \nu} C_{t, \mu \nu}^{\nabla, \nabla}(\mathbf{r})\left[\nabla_{\mu} \nabla_{\nu} D_{t}^{1,1}(\mathbf{r})\right]$ is absent: it vanishes identically as $C_{t, \mu \nu}^{\nabla, \nabla}(\mathbf{r})$ is skew-symmetric under the exchange $\mu \leftrightarrow \nu$, while the object in square brackets is symmetric. The term $\sum_{\mu \nu} C_{t, \mu \nu}^{\nabla, \nabla \sigma}(\mathbf{r})\left[\nabla_{\mu} \nabla_{\nu} D_{t, \kappa}^{1, \sigma}(\mathbf{r})\right]$ vanishes as well for the same reason.

To obtain the new formulation of the functional, we will eliminate the two reducible currents of second order, $C_{t, \mu \nu}^{\nabla, \nabla}(\mathbf{r})$ and $C_{t, \mu \nu \kappa}^{\nabla, \nabla \sigma}(\mathbf{r})$, using Eqs. (96) and (97). Furthermore, we will rewrite two currents of third order, $C_{t}^{(\nabla, \nabla) \nabla}(\mathbf{r})$ and $C_{t}^{(\nabla, \nabla) \nabla \sigma}(\mathbf{r})$ using Eqs. (110) and (103), respectively. Eliminating these four densities, the relevant terms of Eqs. (A1) and (A2) become

$$
\begin{aligned}
&-2 C_{t}^{(4) M \rho} \sum_{\mu \nu} C_{t, \mu \nu}^{\nabla, \nabla}(\mathbf{r}) C_{t, \mu \nu}^{\nabla, \nabla}(\mathbf{r})=-C_{t}^{(4) M \rho} \sum_{\mu \nu}\{ {\left[\nabla_{\mu} C_{t, \nu}^{1, \nabla}(\mathbf{r})\right]^{2}-\left[\nabla_{\mu} C_{t, \nu}^{1, \nabla}(\mathbf{r}]\left[\nabla_{\nu} C_{t, \mu}^{1, \nabla}(\mathbf{r})\right]\right\}, } \\
&-2 C_{t}^{(4) M s} \sum_{\mu \nu \kappa} C_{t, \mu \nu \kappa}^{\nabla, \nabla \sigma}(\mathbf{r}) C_{t, \mu \nu \kappa}^{\nabla, \nabla \sigma}(\mathbf{r})=-C_{t}^{(4) M s} \sum_{\mu \nu}\left\{\left[\nabla_{\mu} C_{t, \nu}^{1, \nabla}(\mathbf{r})\right]^{2}-\left[\nabla_{\mu} C_{t, \nu \kappa}^{1, \nabla \sigma}(\mathbf{r})\right]\left[\nabla_{\nu} C_{t, \mu \kappa}^{1, \nabla \sigma}(\mathbf{r})\right]\right\}, \\
&-4 C_{t}^{(4) M \rho} \mathbf{C}_{t}^{1, \nabla}(\mathbf{r}) \cdot \mathbf{C}_{t}^{(\nabla, \nabla) \nabla}(\mathbf{r})=-C_{t}^{(4) M \rho}\left\{-4 \mathbf{C}_{t}^{1, \nabla}(\mathbf{r}) \cdot \mathbf{C}_{t}^{\Delta, \nabla}(\mathbf{r})+2 \mathbf{C}_{t}^{1, \nabla}(\mathbf{r}) \cdot\left[\Delta \mathbf{C}_{t}^{1, \nabla}(\mathbf{r})\right]\right. \\
&\left.+2 \sum_{\mu \nu} C_{t, \mu}^{1, \nabla}(\mathbf{r})\left[\nabla_{\mu} \nabla_{\nu} C_{t, \nu}^{1, \nabla}(\mathbf{r})\right]\right\}, \\
&-4 C_{t}^{(4) M s} \sum_{\mu \nu} C_{t, \mu \nu}^{1, \nabla \sigma}(\mathbf{r}) C_{t, \mu \nu}^{(\nabla, \nabla) \nabla \sigma}(\mathbf{r})=-C_{t}^{(4) M s} \sum_{\mu \nu}\left\{-4 C_{t, \mu \nu}^{1, \nabla \sigma}(\mathbf{r}) C_{t, \mu \nu}^{\Delta, \nabla \sigma}(\mathbf{r})+2 C_{t, \mu \nu}^{1, \nabla \sigma}(\mathbf{r})\left[\Delta C_{t, \mu \nu}^{1, \nabla \sigma}(\mathbf{r})\right]\right. \\
&\left.+2 \sum_{\kappa} C_{t, \mu \nu}^{1, \nabla \sigma}(\mathbf{r})\left[\nabla_{\mu} \nabla_{\kappa} C_{t, \kappa \nu}^{1, \nabla \sigma}(\mathbf{r})\right]\right\} .
\end{aligned}
$$

Inserting Eqs. (A3)-(A6) into Eqs. (A1) and (A2) leads to an N2LO energy density $\mathcal{E}_{t}^{(4)}(\mathbf{r})$ that is equivalent (but not yet equal) to our formulation of the energy density in Eqs. (117) and (120). To complete the transformation, we note the following integral identities

$$
\begin{aligned}
-\int d^{3} r \sum_{\mu \nu}\left[\nabla_{\mu} C_{t, \nu}^{1, \nabla}(\mathbf{r})\right]^{2} & =+\int d^{3} r \sum_{\mu} C_{t, \mu}^{1, \nabla}(\mathbf{r}) \Delta C_{t, \mu}^{1, \nabla}(\mathbf{r}), \\
-\int d^{3} r \sum_{\mu \nu}\left[\nabla_{\mu} C_{t, \nu}^{1, \nabla}(\mathbf{r})\right]\left[\nabla_{\nu} C_{t, \mu}^{1, \nabla}(\mathbf{r})\right] & =-\int d^{3} r\left[\sum_{\mu} \nabla_{\mu} C_{t, \mu}^{1, \nabla}(\mathbf{r})\right]^{2}, \\
-\int d^{3} r \sum_{\mu \nu \kappa}\left[\nabla_{\mu} C_{t, \nu \kappa}^{1, \nabla \sigma}(\mathbf{r})\right]^{2} & =+\int d^{3} r \sum_{\mu \nu} C_{t, \mu \nu}^{1, \nabla \sigma}(\mathbf{r}) \Delta C_{t, \mu \nu}^{1, \nabla \sigma}(\mathbf{r}), \\
-\int d^{3} r \sum_{\mu \nu \kappa}\left[\nabla_{\mu} C_{t, \nu \kappa}^{1, \nabla \sigma}(\mathbf{r})\right]\left[\nabla_{\nu} C_{t, \mu \kappa}^{1, \nabla \sigma}(\mathbf{r})\right] & =-\int d^{3} r \sum_{\nu}\left[\sum_{\mu} \nabla_{\mu} C_{t, \mu \nu}^{1, \nabla \kappa}(\mathbf{r})\right]^{2} \\
-\int d^{3} r \sum_{\mu \nu} C_{t, \mu}^{1, \nabla}(\mathbf{r})\left[\nabla_{\mu} \nabla_{\nu} C_{t, \nu}^{1, \nabla}(\mathbf{r})\right] & =+\int d^{3} r\left[\nabla \cdot \mathbf{C}_{t}^{1, \nabla}(\mathbf{r})\right]^{2}, \\
-\int d^{3} r \sum_{\mu \nu \kappa} C_{t, \mu \nu}^{1, \nabla \sigma}(\mathbf{r})\left[\nabla_{\mu} \nabla_{\kappa} C_{t, \kappa \nu}^{1, \nabla \sigma}(\mathbf{r})\right] & =+\int d^{3} r \sum_{\nu}\left[\sum_{\mu} \nabla_{\mu} C_{t, \mu \nu}^{1, \nabla \sigma}(\mathbf{r})\right]^{2}
\end{aligned}
$$


which can all be verified easily by partial integration. We are now allowed to replace the integrands on the lefthand-side of Eqs. (A7)-(A12) in the formulation of the energy density by the expressions under the right-hand integral. While these replacements result in a different energy density $\mathcal{E}_{\mathrm{Sk}}^{(4)}$, they do not modify the total energy and hence do not modify the physics of the EDF.

Combining all of the above, we obtain the final form of the N2LO energy density, provided we identify the N2LO coupling constants $A_{\mathrm{t}, \mathrm{e} / \mathrm{o}}^{(4, i)}$ with the coupling constants of Ref. [36] as follows:

$$
\begin{array}{ll}
A_{t, \mathrm{e}}^{(4,1)}=+C_{t}^{(4) \Delta \rho}, & A_{t, \mathrm{o}}^{(4,1)}=+C_{t}^{(4) \Delta s}, \\
A_{t, \mathrm{e}}^{(4,2)}=+C_{t}^{(4) M \rho}, & A_{t, \mathrm{o}}^{(4,2)}=+C_{t}^{(4) M s}, \\
A_{t, \mathrm{e}}^{(4,3)}=+C_{t}^{(4) M \rho}, & A_{t, \mathrm{o}}^{(4,3)}=+C_{t}^{(4) M s}, \\
A_{t, \mathrm{e}}^{(4,4)}=+2 C_{t}^{(4) M \rho}, & A_{t, \mathrm{o}}^{(4,4)}=+2 C_{t}^{(4) M s}, \\
A_{t, \mathrm{e}}^{(4,5)}=-2 C_{t}^{(4) M \rho}, & A_{t, \mathrm{o}}^{(4,5)}=-2 C_{t}^{(4) M s}, \\
A_{t, \mathrm{e}}^{(4,6)}=-C_{t}^{(4) M s}, & A_{t, \mathrm{o}}^{(4,6)}=-C_{t}^{(4) M \rho}, \\
A_{t, \mathrm{e}}^{(4,7)}=-2 C_{t}^{(4) M s}, & A_{t, \mathrm{o}}^{(4,7)}=-2 C_{t}^{(4) M \rho}, \\
A_{t, \mathrm{e}}^{(4,8)}=+4 C_{t}^{(4) M s}, & A_{t, \mathrm{o}}^{(4,8)}=+4 C_{t}^{(4) M \rho} .
\end{array}
$$

\section{Appendix B: Coupling constants}

In the expressions for the Skyrme EDF (115)-(120), we have given each term an individual coupling constant $A_{t, \mathrm{e} / \mathrm{o}}^{(n, i)}$, which is the most straightforward way for the implementation as well as subsequent testing and debugging of an EDF in a numerical code. Not all of these coupling constants, however, necessarily represent an independent degree of freedom of the EDF. While each individual term of the Skyrme EDF defined in Eqs. (115)-(120) is invariant under spatial rotations, translations, space and time inversion of the coordinates used to describe the nucleus, there are a few more subtle invariances that in some cases can only be satisfied by specific combinations of several terms in the EDF, thereby reducing the number of independent coupling constants. The most prominent ones are Galilean invariance, local gauge invariance, and that the EDF is generated by an underlying anti-symmetrized many-body interaction.

Galilean invariance of the EDF, which signifies that only the kinetic energy changes in a specific way when going from one inertial frame of reference to another one, but not the interaction energy 21, 24, 52, 55], is a necessity for any meaningful dynamical EDF calculation. This concerns time-dependent meanfield calculations and their limiting cases such as linear response theory and also cranked mean-field calculations such as the ones reported on in Sec. VE Among the additional symmetries mentioned above, Galilean invariance introduces the smallest set of interdependencies among the coupling constants of the EDF [21].

According to Noether's second theorem, local gauge invariances are not at the origin of conservation laws, but impose constraints on the form of the equations of motion. In the context of nuclear EDF methods, the requirement of local gauge invariance ensures that the continuity equations keep the simple form of Eqs. (121) and (122). It has been pointed out that energy density functionals that are invariant under local gauge transformations are also automatically Galilean invariant 24], but not vice versa. Up to NLO, however, these two symmetries impose the same relations among the coupling constants of the EDF [21, 24]. Beginning with N2LO, one can construct Galilean-invariant terms that are not invariant under arbitrary local gauge transformations anymore.

Another requirement that can be imposed is that the EDF is generated by an underlying effective manybody interaction, which signifies that the EDF has the exchange symmetry of the Pauli principle 57, 58. The majority of parametrizations of the Skyrme NLO EDF does not satisfy such requirement, see for example Refs. [1, 9 11, 18, 45] and references therein for a discussion of motivations for, and consequences of, this practice. In recent years, there are efforts going in the opposite directions of either exploiting the additional freedom of lifting the constraints from an underlying generating operator [6 8], or of respecting those constraints as much as possible at least for the particle-hole part of the EDF, although such EDFs usually allow for density-dependent terms that also might be problematic in this context [58, 85 87]. The necessary Galilean and optional local-gauge invariance of such operator is then automatically transferred to the terms of the EDF that it generates.

The construction of the SN2LO1 parametrization of Ref. [36] has followed the latter strategy: the EDF has been generated from a locally-gauge-invariant 6 effective interaction $\hat{V}_{\mathrm{Sk}}$ of the form of Eq. (2) 29 31, 34 36. In this case, all 56 coupling constants of the Skyrme EDF of Eqs. (115)-120) can be related to the following much smaller set of thirteen parameters of $\hat{V}_{\mathrm{Sk}}$

$$
t_{0}, x_{0}, t_{1}^{(2)}, x_{1}^{(2)}, t_{2}^{(2)}, x_{2}^{(2)}, t_{1}^{(4)}, x_{1}^{(4)}, t_{2}^{(4)}, x_{2}^{(4)}, t_{3}, x_{3}, W_{0} .
$$

The exponent $\alpha$ of the density dependence constitutes an additional parameter of both sets.

As in Ref. [36], the NLO density-dependent interaction $\hat{V}_{\mathrm{NLO}}^{\mathrm{DD}}$ is parameterized by the parameters $t_{3}, x_{3}$, and $\alpha$, while the parameter $W_{0}$ determines the NLO spin-orbit

${ }^{6}$ Note that Eq. (2) only contains the locally-gauge-invariant central terms, but not the tensor terms discussed for example in Refs. [29 33], which explains the presence of only four out of the six possible locally-gauge-invariant terms at N2LO as identified in Ref. [21, 24]. 
interaction $\hat{V}_{\mathrm{NLO}}^{\mathrm{SO}}$. The remaining parameters determine the central part of the interaction $\hat{V}_{\mathrm{N} 2 \mathrm{LO}}^{C}$; this interaction reduces to an NLO Skyrme interaction if the parameters $t_{1}^{(4)}, x_{1}^{(4)}, t_{2}^{(4)}, x_{2}^{(4)}$ are set to zero.

For the coupling constants of the central terms $\hat{V}^{\mathrm{C}}$, it will be useful to use the following shorthand notation

$$
\begin{array}{ll}
C_{00}^{+}(t, x)=+\frac{3}{8} t, & C_{01}^{+}(t, x)=-\frac{1}{8} t-\frac{1}{4} t x, \\
C_{10}^{+}(t, x)=-\frac{1}{8} t+\frac{1}{4} t x, & C_{11}^{+}(t, x)=-\frac{1}{8} t, \\
C_{00}^{-}(t, x)=+\frac{5}{8} t+\frac{1}{2} t x, & C_{01}^{-}(t, x)=+\frac{1}{8} t+\frac{1}{4} t x, \\
C_{10}^{-}(t, x)=+\frac{1}{8} t+\frac{1}{4} t x, & C_{11}^{-}(t, x)=+\frac{1}{8} t .
\end{array}
$$

The $C_{S T}^{\pi}(t, x)$ represent the generic coupling constants that are obtained when evaluating the fully antisymmetrized Hartree-Fock expectation value of a generic central contact interaction 7

$$
\begin{aligned}
& \left\langle\mathrm{HF}\left|\hat{V}_{i}^{\mathrm{C}}\right| \mathrm{HF}\right\rangle \\
& =\left\langle\mathrm{HF}\left|t_{i}\left(1+x_{i} \hat{P}_{12}^{\sigma}\right) \hat{O}^{\pi}\left(\mathbf{r}_{1}, \mathbf{r}_{2}, \mathbf{r}_{1}^{\prime}, \mathbf{r}_{2}^{\prime}\right)\right| \mathrm{HF}\right\rangle \\
& =\iiint \int \mathrm{d}^{3} r_{1} \mathrm{~d}^{3} r_{2} \mathrm{~d}^{3} r_{1}^{\prime} \mathrm{d}^{3} r_{2}^{\prime} \hat{O}^{\pi}\left(\mathbf{r}_{1}, \mathbf{r}_{2} ; \mathbf{r}_{1}^{\prime}, \mathbf{r}_{2}^{\prime}\right) \\
& \quad \times \sum_{t=0,1}\left[C_{0 t}^{\pi}\left(t_{i}, x_{i}\right) \rho_{t}\left(\mathbf{r}_{1}, \mathbf{r}_{1}^{\prime}\right) \rho_{t}\left(\mathbf{r}_{2}, \mathbf{r}_{2}^{\prime}\right)\right. \\
& \left.\quad+C_{1 t}^{\pi}\left(t_{i}, x_{i}\right) \mathbf{s}_{t}\left(\mathbf{r}_{1}, \mathbf{r}_{1}^{\prime}\right) \cdot \mathbf{s}_{t}\left(\mathbf{r}_{2}, \mathbf{r}_{2}^{\prime}\right)\right]
\end{aligned}
$$

where the $\hat{O}^{\pi}\left(\mathbf{r}_{1}, \mathbf{r}_{2}, \mathbf{r}_{1}^{\prime}, \mathbf{r}_{2}^{\prime}\right)$ is the part of the operator of the $i$ th term in the pseudopotential $\hat{V}^{\mathrm{C}}$ that acts in position space and that has the parity $\pi= \pm$ under spatial inversion. For a central contact interaction, this part of the operator is by construction a scalar in coordinate space and does not act in spin or isospin space.

In terms of this shorthand, the expressions for the coupling constants of the time-even LO and NLO terms of the Skyrme functional, Eqs. (115) and (116) are:

$$
\begin{aligned}
& A_{t, \mathrm{e}}^{(0,1)}=+C_{0 t}^{+}\left(t_{0}, x_{0}\right), \\
& A_{t, \mathrm{e}}^{(0,2)}=+\frac{1}{6} C_{0 t}^{+}\left(t_{3}, x_{3}\right), \\
& A_{t, \mathrm{e}}^{(2,1)}=-\frac{3}{8} C_{0 t}^{+}\left(t_{1}^{(2)}, x_{1}^{(2)}\right)+\frac{1}{8} C_{0 t}^{-}\left(t_{2}^{(2)}, x_{2}^{(2)}\right), \\
& A_{t, \mathrm{e}}^{(2,2)}=+\frac{1}{2} C_{0 t}^{+}\left(t_{1}^{(2)}, x_{1}^{(2)}\right)+\frac{1}{2} C_{0 t}^{-}\left(t_{2}^{(2)}, x_{2}^{(2)}\right), \\
& A_{t, \mathrm{e}}^{(2,3)}=-\frac{1}{2} C_{1 t}^{+}\left(t_{1}^{(2)}, x_{1}^{(2)}\right)-\frac{1}{2} C_{1 t}^{-}\left(t_{2}^{(2)}, x_{2}^{(2)}\right), \\
& A_{0, \mathrm{e}}^{(2,4)}=-\frac{3}{4} W_{0}, \\
& A_{1, \mathrm{e}}^{(2,4)}=-\frac{1}{4} W_{0} .
\end{aligned}
$$

The coupling constants for the time-odd terms at LO and NLO are given by

$$
A_{t, \mathrm{o}}^{(0,1)}=+C_{1 t}^{+}\left(t_{0}, x_{0}\right),
$$

\footnotetext{
7 Similar generic combinations can also be constructed for spinorbit and tensor interactions, but for the specific functional discussed here doing so will not further simplify the notation.
}

$$
\begin{aligned}
& A_{t, \mathrm{o}}^{(0,2)}=+\frac{1}{6} C_{1 t}^{+}\left(t_{3}, x_{3}\right), \\
& A_{t, \mathrm{o}}^{(2,1)}=-\frac{3}{8} C_{1 t}^{+}\left(t_{1}^{(2)}, x_{1}^{(2)}\right)+\frac{1}{8} C_{1 t}^{-}\left(t_{2}^{(2)}, x_{2}^{(2)}\right), \\
& A_{t, \mathrm{o}}^{(2,2)}=+\frac{1}{2} C_{1 t}^{+}\left(t_{1}^{(2)}, x_{1}^{(2)}\right)+\frac{1}{2} C_{1 t}^{-}\left(t_{2}^{(2)}, x_{2}^{(2)}\right), \\
& A_{t, \mathrm{o}}^{(2,3)}=-\frac{1}{2} C_{0 t}^{+}\left(t_{1}^{(2)}, x_{1}^{(2)}\right)-\frac{1}{2} C_{0 t}^{-}\left(t_{2}^{(2)}, x_{2}^{(2)}\right), \\
& A_{0, \mathrm{o}}^{(2,4)}=-\frac{3}{4} W_{0}, \\
& A_{1, \mathrm{o}}^{(2,4)}=-\frac{1}{4} W_{0} .
\end{aligned}
$$

The coupling constants appearing in the time-even, N2LO part of the functional $\mathcal{E}_{\text {Sk,e }}^{(4)}$ of Eq. (117), are

$$
\begin{aligned}
& A_{t, \mathrm{e}}^{(4,1)}=+\frac{3}{16} C_{0 t}^{+}\left(t_{1}^{(4)}, x_{1}^{(4)}\right)-\frac{1}{16} C_{0 t}^{-}\left(t_{2}^{(4)}, x_{2}^{(4)}\right), \\
& A_{t, \mathrm{e}}^{(4,2)}=+\frac{1}{4} C_{0 t}^{+}\left(t_{1}^{(4)}, x_{1}^{(4)}\right)+\frac{1}{4} C_{0 t}^{-}\left(t_{2}^{(4)}, x_{2}^{(4)}\right), \\
& A_{t, \mathrm{e}}^{(4,3)}=+\frac{1}{4} C_{0 t}^{+}\left(t_{1}^{(4)}, x_{1}^{(4)}\right)+\frac{1}{4} C_{0 t}^{-}\left(t_{2}^{(4)}, x_{2}^{(4)}\right), \\
& A_{t, \mathrm{e}}^{(4,4)}=+\frac{1}{2} C_{0 t}^{+}\left(t_{1}^{(4)}, x_{1}^{(4)}\right)+\frac{1}{2} C_{0 t}^{-}\left(t_{2}^{(4)}, x_{2}^{(4)}\right), \\
& A_{t, \mathrm{e}}^{(4,5)}=-\frac{1}{2} C_{0 t}^{+}\left(t_{1}^{(4)}, x_{1}^{(4)}\right)-\frac{1}{2} C_{0 t}^{-}\left(t_{2}^{(4)}, x_{2}^{(4)}\right), \\
& A_{t, \mathrm{e}}^{(4,6)}=-\frac{1}{4} C_{1 t}^{+}\left(t_{1}^{(4)}, x_{1}^{(4)}\right)-\frac{1}{4} C_{1 t}^{-}\left(t_{2}^{(4)}, x_{2}^{(4)}\right), \\
& A_{t, \mathrm{e}}^{(4,7)}=-\frac{1}{2} C_{1 t}^{+}\left(t_{1}^{(4)}, x_{1}^{(4)}\right)-\frac{1}{2} C_{1 t}^{-}\left(t_{2}^{(4)}, x_{2}^{(4)}\right), \\
& A_{t, \mathrm{e}}^{(4,8)}=+C_{1 t}^{+}\left(t_{1}^{(4)}, x_{1}^{(4)}\right)+C_{1 t}^{-}\left(t_{2}^{(4)}, x_{2}^{(4)}\right) .
\end{aligned}
$$

We have for the coupling constants appearing in the timeodd N2LO energy density $\mathcal{E}_{\text {Sk,o }}^{(4)}$ of Eq. (120)

$$
\begin{aligned}
& A_{t, \mathrm{o}}^{(4,1)}=+\frac{3}{16} C_{1 t}^{+}\left(t_{1}^{(4)}, x_{1}^{(4)}\right)-\frac{1}{16} C_{1 t}^{-}\left(t_{2}^{(4)}, x_{2}^{(4)}\right), \\
& A_{t, \mathrm{o}}^{(4,2)}=+\frac{1}{4} C_{1 t}^{+}\left(t_{1}^{(4)}, x_{1}^{(4)}\right)+\frac{1}{4} C_{1 t}^{-}\left(t_{2}^{(4)}, x_{2}^{(4)}\right), \\
& A_{t, \mathrm{o}}^{(4,3)}=+\frac{1}{4} C_{1 t}^{+}\left(t_{1}^{(4)}, x_{1}^{(4)}\right)+\frac{1}{4} C_{1 t}^{-}\left(t_{2}^{(4)}, x_{2}^{(4)}\right), \\
& A_{t, \mathrm{o}}^{(4,4)}=+\frac{1}{2} C_{1 t}^{+}\left(t_{1}^{(4)}, x_{1}^{(4)}\right)+\frac{1}{2} C_{1 t}^{-}\left(t_{2}^{(4)}, x_{2}^{(4)}\right), \\
& A_{t, \mathrm{o}}^{(4,5)}=-\frac{1}{2} C_{1 t}^{+}\left(t_{1}^{(4)}, x_{1}^{(4)}\right)-\frac{1}{2} C_{1 t}^{-}\left(t_{2}^{(4)}, x_{2}^{(4)}\right), \\
& A_{t, \mathrm{o}}^{(4,6)}=-\frac{1}{4} C_{0 t}^{+}\left(t_{1}^{(4)}, x_{1}^{(4)}\right)-\frac{1}{4} C_{0 t}^{-}\left(t_{2}^{(4)}, x_{2}^{(4)}\right), \\
& A_{t, \mathrm{o}}^{(4,7)}=-\frac{1}{2} C_{0 t}^{+}\left(t_{1}^{(4)}, x_{1}^{(4)}\right)-\frac{1}{2} C_{0 t}^{-}\left(t_{2}^{(4)}, x_{2}^{(4)}\right), \\
& A_{t, \mathrm{o}}^{(4,8)}=+C_{0 t}^{+}\left(t_{1}^{(4)}, x_{1}^{(4)}\right)+C_{0 t}^{-}\left(t_{2}^{(4)}, x_{2}^{(4)}\right) .
\end{aligned}
$$

A detailed analysis of Galilean invariance of particle-hole terms in the N2LO EDF that is hidden in some of the above relations will be given elsewhere.

\section{Appendix C: Mean-field potentials}

The mean-field potentials associated with the N2LO functional can be obtained in a straight-forward but slightly tedious way by varying the energy with respect to the local normal densities, Eq. (123). As a reference, we include their full expressions for the SN2LO1 parametrization here. Defining a shorthand for the combinations of coupling constants that appear in contributions from the same $\left(q^{\prime}=q\right)$ and the other $\left(q^{\prime} \neq q\right)$ nucleon species

$$
A_{q q^{\prime}, x}^{(i, j)}=\left(A_{0, x}^{(i, j)}+A_{1, x}^{(i, j)}\right) \delta_{q q^{\prime}}+\left(A_{0, x}^{(i, j)}-A_{1, x}^{(i, j)}\right)\left(1-\delta_{q q^{\prime}}\right)
$$




$$
=A_{0, x}^{(i, j)}+\left(2 \delta_{q q^{\prime}}-1\right) A_{1, x}^{(i, j)}
$$

time-even densities are given by

the contribution from the Skyrme EDF to the potentials for the nucleon species $q=p, n$ that are associated with

$$
\begin{aligned}
F_{q}^{1,1}(\mathbf{r})= & \sum_{q^{\prime}=p, n}\left\{2 A_{q q^{\prime}, \mathrm{e}}^{(0,1)} D_{q^{\prime}}^{1,1}(\mathbf{r})+2 A_{q q^{\prime}, \mathrm{e}}^{(0,1)}\left[D_{0}^{1,1}(\mathbf{r})\right]^{\alpha} D_{q^{\prime}}^{1,1}(\mathbf{r})\right. \\
& +2 A_{q q^{\prime}, \mathrm{e}}^{(2,1)}\left[\Delta D_{q^{\prime}}^{1,1}(\mathbf{r})\right]+A_{q q^{\prime} \mathrm{e}}^{(2,2)} D_{q^{\prime}}^{(\nabla, \nabla)}+A_{q q^{\prime}, \mathrm{e}}^{(2,4)}\left(\nabla \cdot \mathbf{C}_{q^{\prime}}^{1, \nabla \times \sigma}\right) \\
& \left.+2 A_{q q^{\prime}, \mathrm{e}}^{(4,1)}\left[\Delta \Delta D_{q^{\prime}}^{1,1}(\mathbf{r})\right]+A_{q q^{\prime}, \mathrm{e}}^{(4,2)} D_{q^{\prime}}^{\Delta, \Delta}(\mathbf{r})+A_{q q^{\prime}, \mathrm{e}}^{(4,5)} \sum_{\mu, \nu}\left[\nabla_{\mu} \nabla_{\nu} D_{q^{\prime}, \mu \nu}^{\nabla, \nabla}(\mathbf{r})\right]\right\} \\
& +\alpha A_{0, \mathrm{e}}^{(0,2)}\left[D_{0}^{1,1}(\mathbf{r})\right]^{\alpha-1}\left[D_{0}^{1,1}(\mathbf{r})\right]^{2}+\alpha A_{1, \mathrm{e}}^{(0,2)}\left[D_{0}^{1,1}(\mathbf{r})\right]^{\alpha-1}\left[D_{1}^{1,1}(\mathbf{r})\right]^{2} \\
& +\alpha A_{0, \mathrm{o}}^{(0,2)}\left[D_{0}^{1,1}(\mathbf{r})\right]^{\alpha-1}\left[\mathbf{D}_{0}^{1, \sigma}(\mathbf{r})\right]^{2}+\alpha A_{1, \mathrm{o}}^{(0,2)}\left[D_{0}^{1,1}(\mathbf{r})\right]^{\alpha-1}\left[\mathbf{D}_{1}^{1, \sigma}(\mathbf{r})\right]^{2}, \\
G_{q, \mu \nu}^{1, \nabla \sigma}(\mathbf{r})= & \sum_{q^{\prime}=p, n}\left\{2 A_{q q^{\prime}, \mathrm{e}}^{(2,3)} C_{q^{\prime}, \mu \nu}^{1, \nabla \sigma}-A_{q q^{\prime}, \mathrm{e}}^{(2,4)} \sum_{\kappa} \epsilon_{\kappa \mu \nu}\left[\nabla_{\kappa} D_{q^{\prime}}^{1,1}(\mathbf{r})\right]\right. \\
& \left.+2 A_{q q^{\prime}, \mathrm{e}}^{(4,6)}\left[\Delta C_{q^{\prime}, \mu \nu}^{1, \nabla \sigma}\right]-2 A_{q q^{\prime}, \mathrm{e}}^{(4,7)} \sum_{\kappa}\left[\nabla_{\mu} \nabla_{\kappa} C_{q^{\prime}, \kappa \nu}^{1, \nabla \sigma}\right]+A_{q q^{\prime}, \mathrm{e}}^{(4,8)} C_{q^{\prime}, \mu \nu}^{\Delta, \nabla \sigma}\right\}, \\
F_{q, \mu \nu}^{\nabla, \nabla}(\mathbf{r})= & \sum_{q^{\prime}=p, n}\left\{A_{q q^{\prime}, \mathrm{e}}^{(2,2)} D_{q^{\prime}}^{1,1}(\mathbf{r}) \delta_{\mu \nu}+2 A_{q q^{\prime}, \mathrm{e}}^{(4,3)} D_{q^{\prime}}^{(\nabla, \nabla)}(\mathbf{r}) \delta_{\mu \nu}+2 A_{q q^{\prime}, \mathrm{e}}^{(4,4)} D_{q^{\prime}, \mu \nu}^{\nabla, \nabla}(\mathbf{r})+A_{q q^{\prime}, \mathrm{e}}^{(4,5)}\left[\nabla_{\mu} \nabla_{\nu} D_{q^{\prime}}^{1,1}(\mathbf{r})\right]\right\}, \\
G_{q, \mu \nu}^{\Delta, \nabla \sigma}(\mathbf{r})= & \sum_{q^{\prime}=p, n} A_{q q^{\prime}, \mathrm{e}}^{(4,8)} C_{q^{\prime}, \mu \nu}^{1, \nabla \sigma}, \\
F_{q}^{\Delta, \Delta}(\mathbf{r})= & \sum_{q^{\prime}=p, n} A_{q q^{\prime}, \mathrm{e}}^{(4,2)} D_{q^{\prime}}^{1,1}(\mathbf{r}),
\end{aligned}
$$

whereas for the potentials associated with time-odd densities one has

$$
\begin{aligned}
\mathbf{F}_{q}^{1, \sigma}(\mathbf{r})= & \sum_{q^{\prime}=p, n}\left\{2 A_{q q^{\prime}, \mathrm{o}}^{(0,1)} \mathbf{D}_{q^{\prime}}^{1, \sigma}(\mathbf{r})+2 A_{q q^{\prime}, \mathrm{o}}^{(0,2)}\left[D_{0}^{1,1}(\mathbf{r})\right]^{\alpha} \mathbf{D}_{q^{\prime}}^{1, \sigma}(\mathbf{r})\right. \\
& +2 A_{q q^{\prime}, \mathrm{o}}^{(2,1)}\left[\Delta \mathbf{D}_{q^{\prime}}^{1, \sigma}(\mathbf{r})\right]+A_{q q^{\prime}, \mathrm{o}}^{(2,2)} \mathbf{D}_{q^{\prime}}^{(\nabla, \nabla) \sigma}(\mathbf{r})+A_{q q^{\prime}, \mathrm{o}}^{(2,4)}\left[\boldsymbol{\nabla} \times \mathbf{C}_{q^{\prime}}^{1, \nabla}(\mathbf{r})\right] \\
& \left.+2 A_{q q^{\prime}, \mathrm{o}}^{(4,1)}\left[\Delta \Delta \mathbf{D}_{q^{\prime}}^{1, \sigma}(\mathbf{r})\right]+A_{q q^{\prime}, \mathrm{o}}^{(4,2)} \mathbf{D}_{q^{\prime}}^{\Delta, \Delta \sigma}(\mathbf{r})+A_{q q^{\prime}, \mathrm{o}}^{(4,5)} \sum_{\mu \nu \kappa}\left[\nabla_{\mu} \nabla_{\nu} D_{q^{\prime}, \mu \nu \kappa}^{\nabla, \nabla \sigma}(\mathbf{r})\right] \mathbf{e}_{\kappa}\right\}, \quad(\mathrm{C} 7), \quad(\mathrm{C} 8), \\
\mathbf{G}_{q}^{1, \nabla}(\mathbf{r})= & \sum_{q^{\prime}=p, n}\left(2 A_{q q^{\prime}, \mathrm{o}}^{(2,3)} \mathbf{C}_{q^{\prime}}^{1, \nabla}(\mathbf{r})+A_{q q^{\prime}, \mathrm{o}}^{(2,4)}\left[\nabla \times \mathbf{D}_{q^{\prime}}^{1, \sigma}(\mathbf{r})\right]\right. \\
& \left.+2 A_{q q^{\prime}, \mathrm{o}}^{(4,6)}\left[\Delta \mathbf{C}_{q^{\prime}}^{1, \nabla}(\mathbf{r})\right]+2 A_{q q^{\prime}, \mathrm{o}}^{(4,7)}\left\{\nabla\left[\nabla \cdot \mathbf{C}_{q^{\prime}}^{1, \nabla}(\mathbf{r})\right]\right\}+A_{q q^{\prime}, \mathrm{o}}^{(4,8)} \mathbf{C}_{q^{\prime}}^{\Delta, \nabla}(\mathbf{r})\right), \\
F_{q, \mu \nu \kappa}^{\nabla, \nabla \sigma}(\mathbf{r})= & \sum_{q^{\prime}=p, n}\left\{A_{q q^{\prime}, \mathrm{o}}^{(2,2)} D_{q^{\prime}, \kappa}^{1, \sigma}(\mathbf{r}) \delta_{\mu \nu}+2 A_{q q^{\prime}, \mathrm{o}}^{(4,3)} D_{q^{\prime}, \kappa}^{(\nabla, \nabla) \sigma}(\mathbf{r}) \delta_{\mu \nu}+2 A_{q q^{\prime}, \mathrm{o}}^{(4,4)} D_{q^{\prime}, \mu \nu \kappa}^{\nabla, \nabla \sigma}(\mathbf{r})+A_{q q^{\prime}, \mathrm{o}}^{(4,5)}\left[\nabla_{\mu} \nabla_{\nu} D_{q^{\prime}, \mu \nu \kappa}^{1, \sigma}(\mathbf{r})\right]\right\}, \\
\mathbf{G}_{q}^{\Delta, \nabla}(\mathbf{r})= & \sum_{q^{\prime}=p, n} A_{q q^{\prime}, \mathrm{o}}^{(4,8)} \mathbf{C}_{q^{\prime}}^{1, \nabla}(\mathbf{r}), \\
\mathbf{F}_{q}^{\Delta, \Delta \sigma}(\mathbf{r})= & \sum_{q^{\prime}=p, n} A_{q q^{\prime}, \mathrm{o}}^{(4,2)} \mathbf{D}_{q^{\prime}}^{1, \sigma}(\mathbf{r}),
\end{aligned}
$$


energy that are also contained in Eq. (1) still have to be added to these expressions.

\section{Appendix D: Point-group transformations and point-group symmetries of local densities}

In what follows, we will sketch how the symmetries of single-particle states, of the densities as defined in Sec. III and of the corresponding potentials as defined in Sections IVC and IVE can be used to reduce the numerical cost of symmetry-restricted HFB calculations with N2LO EDFs. We will concentrate on symmetries under the point-group transformation of the coordinate system as defined in Ref. [48] that provides the symmetries that are of relevance in a $3 \mathrm{~d}$ Cartesian representation. Elements $\hat{U}$ of that group that are relevant for our discussion are parity $\hat{P}$, the signature operators $\hat{R}_{\mu}=e^{i \pi \hat{J}_{\mu}}$, the simplex operators $\hat{S}_{\mu}=\hat{R}_{\mu} \hat{P}$, all of which are linear, and the anti-linear time-reversal operator $\check{T}$. Note that the properties of these operators and the corresponding group are slightly different when applied in the spaces of many-body states representing systems with even or odd particle number, and also in the space of single-particle states [48]. We will only need the latter for our discussion.

The point-group transformations can be used to set up symmetry-restricted HFB calculations. To this end, the quasi-particle vacuum is constructed such that the single-particle states

$$
\Psi_{k}(\mathbf{r})=\left(\begin{array}{l}
\operatorname{Re}\left\{\psi_{k}(\mathbf{r},+)\right\}+\mathrm{i} \operatorname{Im}\left\{\psi_{k}(\mathbf{r},+)\right\} \\
\operatorname{Re}\left\{\psi_{k}(\mathbf{r},-)\right\}+\mathrm{i} \operatorname{Im}\left\{\psi_{k}(\mathbf{r},-)\right\}
\end{array}\right)
$$

are eigenstates of some transformation operators. For discrete point-group symmetries as considered here, the group elements can be directly used as symmetry operators. Not every combination of point-group transformations can be chosen as conserved symmetries, though 88]: the maximal set comprises a linear hermitian, a linear anti-hermitian, an anti-linear hermitian and an anti-linear anti-hermitian operator that have to fulfill specific commutation relations [41].

The action of the basic point-group operators on the spinor components can be summarized as

$$
\begin{aligned}
& \psi_{k}^{\hat{P}}(x, y, z, \sigma)=\psi_{k}(-x,-y,-z, \sigma), \\
& \psi_{k}^{\check{T}}(x, y, z, \sigma)=-\sigma \psi_{k}^{*}(x, y, z,-\sigma),
\end{aligned}
$$

$$
\begin{aligned}
& \psi_{k}^{\hat{R}_{x}}(x, y, z, \sigma)=-\mathrm{i} \psi_{k}(x,-y,-z,-\sigma), \\
& \psi_{k}^{\hat{R}_{y}}(x, y, z, \sigma)=\sigma \psi_{k}(-x, y,-z,-\sigma), \\
& \psi_{k}^{\hat{R}_{z}}(x, y, z, \sigma)=-\mathrm{i} \sigma \psi_{k}(-x,-y, z, \sigma),
\end{aligned}
$$

from which the behavior of the four real functions needed to represent a single-particle state (D1) under pointgroup transformations can be easily deduced. For all calculations reported in this paper, we assumed that single-particle states are eigenstates of a subset of the following operators: (linear hermitian) parity $\hat{P}$, (linear anti-hermitian) $z$ signature $\hat{R}_{z}$, and the (anti-linear hermitian) time $y$ simplex $\check{S}_{y}^{T}$

$$
\begin{aligned}
& \hat{P} \Psi_{k}(\mathbf{r})=\pi_{k} \Psi_{k}(\mathbf{r}), \\
& \hat{R}_{z} \Psi_{k}(\mathbf{r})=\mathrm{i} \eta_{k} \Psi_{k}(\mathbf{r}), \\
& \check{S}_{y}^{T} \Psi_{k}(\mathbf{r})=\check{T} \hat{P} \hat{R}_{y} \Psi_{k}(\mathbf{r})=+\Psi_{k}(\mathbf{r}),
\end{aligned}
$$

where $\pi_{k}= \pm 1$ and $\eta_{k}= \pm 1$ are the parity and signature quantum numbers 8 The relation for $\breve{S}_{y}^{T}$ establishes a spatial symmetry, but because of its anti-linearity there is no associated quantum number. Relation (D9) for $\check{S}_{y}^{T}$, however, fixes the relative phase between the singleparticle states [88].

In addition, for some of the calculations reported in Sec. $[\mathrm{V}$ we impose time-reversal symmetry. As an antilinear anti-hermitian operator, $\check{T}$ does not provide an eigenvalue equation, but instead establishes a relation between two single-particle states in the canonical basis that we will label as $k$ and $\bar{k}$ as customarily done

$$
\Psi_{\bar{k}}(\mathbf{r})=\Psi_{k}^{\check{T}}(\mathbf{r})=\check{T} \Psi_{k}(\mathbf{r})
$$

meaning that only one out of the two states needs to be explicitly calculated and kept in storage. (When time-reversal symmetry is broken, the time-reversed of a given state in the canonical basis is in general not a state in the canonical basis, but a superposition of such states.) As time-reversal changes the sign of the imaginary eigenvalues of linear anti-hermitian operators, the states to be treated explicitly can be chosen as the single-particle states for which the signature quantum number $\eta_{k}$ is positive. Note that applying time-reversal twice on a Fermionic state yields the negative of the original state, $\check{T}^{2}=-1$, such that one has $\Psi_{k}(\mathbf{r})=$ $-\widetilde{T} \Psi_{\bar{k}}(\mathbf{r})$.

From Eqs. (D2)-(D6) follows that the four real functions out of which the eigenstates of Eqs. (D7)-(D9) are built satisfy the relations

$$
\operatorname{Re}\left\{\psi_{k}(x, y, z,+)\right\}=+\operatorname{Re}\left\{\psi_{k}(x,-y, z,+)\right\}=+\eta_{k} \operatorname{Re}\left\{\psi_{k}(-x,-y, z,+)\right\}=p_{k} \operatorname{Re}\left\{\psi_{k}(-x,-y,-z,+)\right\}
$$

\footnotetext{
${ }^{8}$ Note that there is an alternative convention where the factor $-\mathrm{i}$ is not taken out from the eigenvalue, such that the single-particle
} 


$$
\begin{aligned}
& \operatorname{Im}\left\{\psi_{k}(x, y, z,+)\right\}=-\operatorname{Im}\left\{\psi_{k}(x,-y, z,+)\right\}=+\eta_{k} \operatorname{Im}\left\{\psi_{k}(-x,-y, z,+)\right\}=p_{k} \operatorname{Im}\left\{\psi_{k}(-x,-y,-z,+)\right\} \\
& \operatorname{Re}\left\{\psi_{k}(x, y, z,-)\right\}=+\operatorname{Re}\left\{\psi_{k}(x,-y, z,-)\right\}=-\eta_{k} \operatorname{Re}\left\{\psi_{k}(-x,-y, z,-)\right\}=p_{k} \operatorname{Re}\left\{\psi_{k}(-x,-y,-z,-)\right\} \\
& \operatorname{Im}\left\{\psi_{k}(x, y, z,-)\right\}=-\operatorname{Im}\left\{\psi_{k}(x,-y, z,-)\right\}=-\eta_{k} \operatorname{Im}\left\{\psi_{k}(-x,-y, z,-)\right\}=p_{k} \operatorname{Im}\left\{\psi_{k}(-x,-y,-z,-)\right\} .
\end{aligned}
$$

From suitable combinations of these one can then construct the symmetries of each of the four real functions under plane reflection as detailed in Table \. These imply that for eigenstates of $\left\{\hat{P}, \hat{R}_{z}, \check{S}_{y}^{T}\right\}$ it is sufficient to have a numerical representation of the singleparticle states in $1 / 8$ of the box. The same choice has been used earlier in Refs. [11, 45, 75].

The same triaxial shape degrees of freedom can also be described choosing signature and time-simplex operators that refer to other cartesian directions [88]. For reasons that ultimately are related to the special role played by the $z$ direction for the quantization of spin and orbital angular momenta, choosing a different set of pointgroup symmetries to describe states with triaxial shapes leads in general to different symmetries of the four real functions needed to represent the single-particle states, and will also lead to different symmetries for some of the local densities.

Among all possible choices, however, ours has the advantage that each of the four functions needed to represent a single-particle state (D1) individually takes three plane reflection symmetries. This is specific to working with eigenstates of $\hat{R}_{z}$ and $\check{S}_{y}^{T}$. The other signature and time-simplex all establish relations between different spinor components and/or their real and imaginary parts, see Eqs. (D2)-(D6). Choosing $\left\{\hat{P}, \hat{R}_{x}, \check{S}_{y}^{T}\right\}$ instead, as done in many other implementations of triaxial HFB [88], leads to a situation where the continuation of each of the four functions needed to represent $\Psi_{k}(\mathbf{r})$ into other octants is provided by a different one among these functions.

The symmetry properties of the single-particle states are then transferred on to the two-body and manybody states, the local densities and the local potentials, meaning that the numerical representation can be limited to some sector of the full volume in which the nucleus is placed, and the information about the eliminated sectors is reconstructed through the conserved symmetries [11, 45, 51, 88.

Tables II and III detail the behavior of all cartesian

TABLE I. Plane reflection symmetries of the real and imaginary parts of the upper and lower spinor components of eigenstates of $\left\{\hat{P}, \hat{R}_{z}, S_{y}^{T}\right\}$ (see text).

\begin{tabular}{lccc}
\hline & $(-x, y, z)$ & $(x,-y, z)$ & $(x, y,-z)$ \\
\hline $\operatorname{Re}\left\{\psi_{k}(\mathbf{r},+)\right\}$ & $+\eta_{k}$ & + & $+\eta_{k} p_{p}$ \\
$\operatorname{Im}\left\{\psi_{k}(\mathbf{r},+)\right\}$ & $-\eta_{k}$ & - & $+\eta_{k} p_{k}$ \\
$\operatorname{Re}\left\{\psi_{k}(\mathbf{r},-)\right\}$ & $-\eta_{k}$ & + & $-\eta_{k} p_{k}$ \\
$\operatorname{Im}\left\{\psi_{k}(\mathbf{r},-)\right\}$ & $+\eta_{k}$ & - & $-\eta_{k} p_{k}$ \\
\hline
\end{tabular}

components of the normal time-even densities entering our choice of representation of the N2LO Skyrme EDF of Eqs. 115) -120) under plane reflection reflection in the three cartesian directions. These are obtained writing out the expressions of Eqs. (71)-(74) in terms of the four real functions on the right-hand-side of Eq. (D1) and using that the component $\nabla_{\mu}$ of the nabla operator changes sign when replacing $r_{\mu}$ by $-r_{\mu}$. For example, having the values of the $x$-component of the current $C_{q, x}^{\Delta, \nabla}(\mathbf{r})$ in the octant where $x, y$, and $z$ are all positive, from Table III it can be deduced that its values in the three adjacent octants are obtained as

$$
\begin{aligned}
& C_{q, x}^{\Delta, \nabla}(-x, y, z)=+C_{q, x}^{\Delta, \nabla}(x, y, z), \\
& C_{q, x}^{\Delta, \nabla}(x,-y, z)=-C_{q, x}^{\Delta, \nabla}(x, y, z), \\
& C_{q, x}^{\Delta, \nabla}(x, y,-z)=+C_{q, x}^{\Delta, \nabla}(x, y, z) .
\end{aligned}
$$

The value of $C_{q, x}^{\Delta, \nabla}(\mathbf{r})$ in the other four octants can be obtained from suitable combinations of these relations. Note that the Tables with the point-group symmetries of normal densities provided by Ref. [48] represent something different: they represent the sign change of a given density when going to the transformed coordinate system, and the entries in the tables provided there can only used for the actually conserved symmetries of the many-body states. By contrast, our Tables represent the continuation of the densities when going from one octant to another in the same coordinate system. None of the plane reflection symmetries listed in Tables II and III corresponds to a conserved symmetry of the actual singleparticle wave functions and therefore the properties of the functions might disagree with the symmetries of densities under a simplex transformation $\hat{S}_{\mu}$ as given in Ref. [48].

TABLE II. Plane reflection symmetries of the time-even normal densities defined in Sec. IIID when working with eigenstates of $\left\{\hat{P}, \hat{R}_{z}, \breve{S}_{y}^{T}\right\}$ (see text).

\begin{tabular}{lcccc}
\hline \hline Density & $\mu \nu$ & $(-x, y, z)$ & $(x,-y, z)$ & $(x, y,-z)$ \\
\hline$D_{q}^{1,1}(\mathbf{r}), D_{q}^{\Delta, \Delta}(\mathbf{r})$ & & + & + & + \\
\hline \multirow{4}{*}{} & $x x, y y, z z$ & + & + & + \\
$D_{q, \mu \nu}^{\nabla, \nabla}(\mathbf{r})$ & $x y, y x$ & - & - & + \\
& $x z, z x$ & - & + & - \\
& $y z, z y$ & + & - & - \\
\hline \multirow{2}{*}{$C_{q, \mu \nu}^{1, \nabla \sigma}(\mathbf{r})$} & $x x, y y, z z$ & - & - & - \\
$C_{q, \mu \nu}^{\Delta, \nabla \sigma}(\mathbf{r})$ & $x y, y x$ & + & + & - \\
& $x z, z x$ & + & - & + \\
\hline \hline
\end{tabular}


TABLE III. Same as Tab. II but for time-odd normal densities.

\begin{tabular}{|c|c|c|c|c|}
\hline Density & $\mu \nu$ & $(-x, y, z)$ & $(x,-y, z)$ & $(x, y,-z)$ \\
\hline \multirow{3}{*}{$\begin{array}{l}D_{q, \mu}^{1, \sigma}(\mathbf{r}) \\
D_{q, \mu}^{\Delta, \Delta \sigma}(\mathbf{r})\end{array}$} & $x$ & - & + & - \\
\hline & $y$ & + & - & - \\
\hline & $z$ & + & + & + \\
\hline \multirow{12}{*}{$D_{q, \mu \nu \kappa}^{\nabla, \nabla \sigma}(\mathbf{r})$} & $x x x, y y x, z z x$, & - & + & - \\
\hline & $x x y, y y y, z z y$ & + & - & - \\
\hline & $x x z, y y z, z z z$ & + & + & + \\
\hline & $x y x, y x x$ & + & - & - \\
\hline & $x y y, y x y$ & - & + & - \\
\hline & $x y z, y x z$ & - & - & + \\
\hline & $x z x, z x x$ & + & + & + \\
\hline & $x z y, z x y$ & - & - & + \\
\hline & $x z z, z x z$ & - & + & - \\
\hline & $y z x, z y x$ & - & - & + \\
\hline & $y z y, z y y$ & + & + & + \\
\hline & $y z z, z y z$ & + & - & - \\
\hline \multirow{3}{*}{$\begin{array}{l}C_{q, \mu}^{1, \nabla}(\mathbf{r}) \\
C_{q, \mu}^{\Delta, \nabla}(\mathbf{r})\end{array}$} & $x$ & + & - & + \\
\hline & $y$ & - & + & + \\
\hline & $z$ & - & - & - \\
\hline
\end{tabular}

TABLE IV. Same as Tab. II but for local pair densities that are time-even in a gauge where $\kappa$ is real. The reflection symmetries of the real and imaginary parts of these possibly complex pair densities are the same.

\begin{tabular}{lcccc}
\hline \hline Density & $\mu \nu$ & $(-x, y, z)$ & $(x,-y, z)$ & $(x, y,-z)$ \\
\hline$\tilde{D}_{q}^{1,1}(\mathbf{r}), \tilde{D}_{q}^{\Delta, \Delta}(\mathbf{r})$ & & + & + & + \\
\hline \multicolumn{5}{c}{} \\
$\tilde{D}_{q, \mu \nu}^{\nabla, \nabla}(\mathbf{r})$ & $x x, y y, z z$ & + & + & + \\
& $x y, y x$ & - & - & + \\
& $x z, z x$ & - & + & - \\
& $y z, z y$ & + & - & - \\
$\tilde{C}_{q, \mu \nu}^{1, \nabla \sigma}(\mathbf{r})$ & $x x, y y, z z$ & - & - & - \\
$\tilde{C}_{q, \mu \nu}^{\Delta, \nabla \sigma}(\mathbf{r})$ & $x y, y x$ & + & + & - \\
& $x z, z x$ & + & - & + \\
\hline \hline
\end{tabular}

Tables IV and $\mathrm{V}$ provide the same information for the time-even pair and time-odd pair densities, respectively. When time-reversal is a conserved symmetry, all densities listed in Tables III and $\mathrm{V}$ are strictly zero.

The energy density $\mathcal{E}(\mathbf{r})$ is a scalar under all spatial similarity transformations, meaning that it has to have the same symmetries as the local normal matter density $D_{q}^{1,1}(\mathbf{r})$. This necessitates that each individual term in Eqs. (115) - (120) is the product of two densities (or of a density and the derivative of a density) that have the same behavior under all plane reflection symmetries. From Eq. (125) follows then that the potentials $F_{q, a}(r)$ take exactly the same symmetries as the density $\mathrm{R}_{q, \mathrm{a}}(\mathbf{r})$ they are associated with.
TABLE V. Same as Tab. II but for local pair densities that are time-odd in a gauge where $\kappa$ is real. Again, the reflection symmetries of the real and imaginary parts of these possibly complex pair densities are the same.

\begin{tabular}{lcccc}
\hline \hline Density & $\mu \nu$ & $(-x, y, z)$ & $(x,-y, z)$ & $(x, y,-z)$ \\
\hline$\tilde{C}_{q}^{1,1}(\mathbf{r}), \tilde{C}_{q}^{\Delta, \Delta}(\mathbf{r})$ & & - & - & + \\
\hline \multicolumn{5}{c}{} \\
$\tilde{C}_{q, \mu \nu}^{\nabla, \nabla}(\mathbf{r})$ & $x x, y y, z z$ & - & - & + \\
& $x y, y x$ & + & + & + \\
& $x z, z x$ & + & - & - \\
& $y z, z y$ & - & + & - \\
\hline \multirow{5}{*}{$\tilde{D}_{q, \mu \nu}^{1, \nabla \sigma}(\mathbf{r})$} & $x x, y y, z z$ & + & + & - \\
$\tilde{D}_{q, \mu \nu}^{\Delta, \nabla \sigma}(\mathbf{r})$ & $x y, y x$ & - & - & - \\
& $x z, z x$ & - & + & + \\
\hline \hline
\end{tabular}

When breaking parity among the symmetries discussed above, such that only $\left\{\hat{R}_{z}, \breve{S}_{y}^{T}\right\}$ remain conserved, one loses the reflection symmetry in the $z=0$ plane in Tables [V] Keeping parity conserved while breaking one of the other spatial symmetries, however, will lead to a situation with a point reflection symmetry instead of plane reflection symmetries that cannot be deduced from these Tables anymore.

The calculations of $\mathrm{Kr}$ and $\mathrm{Nd}$ isotopes discussed in Sec. V were performed imposing the full $\left\{\hat{P}, \hat{R}_{z}, \check{S}_{y}^{T}, \check{T}\right\}$ symmetry on the single-particle states. This corresponds to time-reversal invariant triaxial many-body states, i.e. states whose densities take three plane reflection symmetries, and which necessarily have an average angular momentum of zero, see Ref. [45] for a detailed discussion of this choice. The calculations of the SD band of ${ }^{194} \mathrm{Hg}$ used the single-particle symmetries $\left\{\hat{P}, \hat{R}_{z}, \breve{S}_{y}^{T}\right\}$ instead, which also corresponds to triaxial many-body states, but the broken time-reversal invariance now allows for finite average angular momentum in $z$ direction $\left\langle\hat{J}_{z}\right\rangle$, see for example [11, 89] for further discussion of this choice. Finally, the fission barrier of ${ }^{240} \mathrm{Pu}$ was calculated with conserved $\left\{\hat{R}_{z}, \breve{S}_{y}^{T}, \breve{T}\right\}$, where broken parity lifts one of the plane reflection symmetries of the densities and allows for describing reflection-asymmetric shapes.

\section{Appendix E: Properties of local pair densities that motivate our definitions}

In the following, we further detail the motivations behind our choice of local pair densities as defined in Eqs. (75)- (78).

Despite usually being evaluated for densities obtained from a symmetry-breaking reference state, any meaningful EDF nevertheless has to be constructed such that it is invariant under all symmetry transformations that correspond to the empirical conservation laws of nuclear physics. Among these, most relevant for 
the purpose of defining local pair densities are global gauge transformations. Global gauge-invariance is the global symmetry related to the conservation of particle number [90]. When not mixing protons and neutrons, the many-body state has two separate global gauge invariances $\mathrm{U}(1)_{N} \times \mathrm{U}(1)_{Z}$, one with respect to gauge rotation of neutrons, the other with respect to gauge rotations of protons [90].

A global U(1) gauge transformation $e^{\mathrm{i} \varphi \hat{N}}$ changes the phase of every single-particle state by the angle $\varphi$. On the one hand, the gauge transformation of a given manybody state $|\Phi\rangle$ can be represented through the change of the phase of all single-particle wave functions $\Psi_{k}(\mathbf{r}) \rightarrow$ $\Psi_{k}(\mathbf{r}) e^{\mathrm{i} \varphi}$, while the creation and annihilation operators $\hat{a}_{k}^{\dagger}$ and $\hat{a}_{k}$ still refer then to the original single-particle basis. On the other hand, as an alternative one can keep the single-particle wave functions unchanged and transform the corresponding creation and annihilation operators instead

$$
\begin{aligned}
& e^{+\mathrm{i} \varphi \hat{N}} \hat{a}_{k} e^{-\mathrm{i} \varphi \hat{N}}=\hat{a}_{k} e^{-\mathrm{i} \varphi}, \\
& e^{+\mathrm{i} \varphi \hat{N}} \hat{a}_{k}^{\dagger} e^{-\mathrm{i} \varphi \hat{N}}=\hat{a}_{k}^{\dagger} e^{+\mathrm{i} \varphi},
\end{aligned}
$$

such that the phase factors are treated as separate entities throughout the calculation. For formal and computational reasons that will become clear in what follows, the latter convention for gauge transformations is more practical when considering the definition of local pair densities.

As we are interested here in the transformations of density matrices (7) and (8) for a given particle species $q$, we will limit our discussion to the global gauge transformation of the auxiliary Bogoliubov quasiparticle vacuum for one generic nucleon species.

In static calculations and when conserving an antilinear anti-hermitian symmetry [41], the amplitudes $v_{k}$ and $u_{k}$ can be chosen to be real in the canonical basis when not mixing protons and neutrons. This is the choice that is usually made in numerical codes, but it is not a necessity. For such specific choice of canonical basis, the corresponding quasiparticle vacuum can be written in the simple BCS form

$$
|\Phi(0)\rangle \equiv \prod_{f} \hat{a}_{f}^{\dagger} \prod_{\substack{k>0 \\ k, k \neq f}}\left(u_{k}+v_{k} \hat{a}_{k}^{\dagger} \hat{a}_{\bar{k}}^{\dagger}\right)|-\rangle,
$$

where $f$ labels completely filled single-particle states and $k$ the paired ones [2]. A gauge-rotated quasiparticle vacuum $|\Phi(\varphi)\rangle$ is then obtained as [57]

$$
\begin{aligned}
|\Phi(\varphi)\rangle & \equiv e^{\mathrm{i} \varphi \hat{N}}|\Phi(0)\rangle \\
& =\prod_{f} \hat{a}_{f}^{\dagger} e^{\mathrm{i} \varphi} \prod_{\substack{k>0 \\
k, k \neq f}}\left(u_{k}+v_{k} e^{2 \mathrm{i} \varphi} \hat{a}_{k}^{\dagger} \hat{a}_{\bar{k}}^{\dagger}\right)|-\rangle .
\end{aligned}
$$

As paired Bogoliubov quasiparticle vacua are not eigenstates of particle number, the global gauge rotation does not simply correspond to a change of phase of the many-body state by $\varphi N_{0}$ as it would be the case for a Slater determinant and any other eigenstate of the particle-number operator $\hat{N}$ with eigenvalue $N_{0}$. Also, there are no simple transformation rules for Bogoliubov quasiparticle creation and annihilation operators, as these mix single-particle creation and annihilation operators that according to Eqs. (E1) and (E2) transform differently.

From the relations (E1) and E2 follows that the normal and anomalous density matrices in some singleparticle basis transform as

$$
\begin{aligned}
\rho_{k \ell}^{\varphi \varphi} & =\left\langle\Phi(\varphi)\left|\hat{a}_{\ell}^{\dagger} \hat{a}_{k}\right| \Phi(\varphi)\right\rangle=\rho_{k \ell}^{00}, \\
\kappa_{k \ell}^{\varphi \varphi} & =\left\langle\Phi(\varphi)\left|\hat{a}_{\ell} \hat{a}_{k}\right| \Phi(\varphi)\right\rangle=\kappa_{k \ell}^{00} e^{+2 \mathrm{i} \varphi}, \\
\kappa_{k \ell}^{\varphi \varphi *} & =\left\langle\Phi(\varphi)\left|\hat{a}_{k}^{\dagger} \hat{a}_{\ell}^{\dagger}\right| \Phi(\varphi)\right\rangle=\kappa_{k \ell}^{00 *} e^{-2 \mathrm{i} \varphi},
\end{aligned}
$$

when changing the gauge of the quasiparticle vacuum (E3) they are constructed from. These relations imply that the choice of a real anomalous density matrix $\kappa_{k \ell}^{*}=\kappa_{k \ell}$ requires a specific choice for the global gauge angle $\varphi$. By contrast, the normal one-body density matrix is not affected by a global gauge transformation of the many-body state it is constructed from, which implies that all local normal densities as defined in Eqs. (60)-63) are automatically invariant under global gauge transformations. Even when working with symmetries and a gauge that permit $\kappa^{*}=\kappa$, the distinction between these two matrices should be kept throughout the formalism as it distinguishes the independent degrees of freedom when deriving the HFB equation, see Sec. IVE,

Yet another point to be taken into consideration is that the definition of local densities has to ensure that any possible symmetry of the single-particle wave functions translates into a symmetry of the densities. This is a pre-requisite for using such symmetries to reduce the numerical cost of calculations in a symmetry-restricted HFB code as explained in Appendix D. When doing so, the numerical representation is limited to some sector of the full volume in which the nucleus is placed, and the information about the sectors eliminated from the numerical representation is reconstructed through the conserved symmetries. From a practical point of view, in a coordinate-space representation this particularly concerns the calculation of numerical derivatives that are to be calculated with different expressions when the function they are applied to is even or odd under some reflection symmetry [45]. Similarly, the conserved symmetries are then used to determine a priori that integrals over products of functions that have different symmetries are zero. That such integrals vanish exactly can in most cases not be deduced from numerically summing up the integrand in the restricted volume; instead, the integral being zero usually results from the cancellation between contributions of same absolute value but different sign from the various sectors of space that are connected by the symmetries. 
For normal densities defined along the lines of Eqs. (60)- (63), the symmetries of the single-particle wave functions are indeed automatically transferred to the densities, which follows from the hermiticity of normal density matrices. For pair densities, the situation is different as the non-local pair densities defined through Eqs. (21) and (22) transform as tensors of rank 2, as do the objects they are calculated from these relations. This has a number of consequences for our discussion. For example, the analysis of their behavior under similarity transformations in space-time reveals that the real and imaginary parts of the two-body wave function $\tilde{\varrho}_{j k}\left(\mathbf{r}^{\prime}, \mathbf{r}\right)$ and $\tilde{\varsigma}_{j k, \mu}\left(\mathbf{r}^{\prime}, \mathbf{r}\right)$ in general transform differently, which should not be a surprise as the same is found for the real and imaginary parts of the upper and lower components of single-particle wave functions, see the discussion in Appendix D, For $\tilde{\varrho}_{j k}\left(\mathbf{r}^{\prime}, \mathbf{r}\right)$ this can be seen from the symmetries of $\tilde{D}_{q}^{1,1}(\mathbf{r})$ and $\tilde{C}_{q}^{1,1}(\mathbf{r})$ as given in Tables IV and $\mathrm{V}$ in Appendix $\mathrm{D}$, as these densities are simply a weighted sum of its real and imaginary parts at $\mathbf{r}=\mathbf{r}^{\prime}$.

As argued above, objects that have different spatial symmetries need to be represented separately in a symmetry-restricted HFB code. This, however, cannot be done in a transparent manner in the traditional notation of local pair densities, i.e. using $\tilde{\rho}_{q}(\mathbf{r}), \tilde{\tau}_{q}(\mathbf{r})$, $\tilde{J}_{q, \mu \nu}(\mathbf{r})$, etc, when $\kappa_{i j}$ is complex: the symmetries of real and imaginary parts of $\tilde{\varrho}_{j k}(\mathbf{r}, \mathbf{r})$ and $\tilde{\varsigma}_{j k, \mu}(\mathbf{r}, \mathbf{r})$ are lost when calculating the real and imaginary parts of these pair densities. This becomes different when separating the traditional pair densities into a $\tilde{D}$ and a $\tilde{C}$ object that only depend on either the real or the imaginary part of $\tilde{\varrho}_{j k}(\mathbf{r}, \mathbf{r})$ and $\tilde{\varsigma}_{j k, \mu}(\mathbf{r}, \mathbf{r})$ as done in the definitions of Eqs. (75)- (78). While the $\tilde{D}$ and a $\tilde{C}$ might still be complex, their real and imaginary parts now have the same spatial symmetries.

Under a global gauge transformation of the reference state, the local pair densities as defined in Eqs. (75) - (78) transform as

$$
\begin{aligned}
\tilde{D}_{q}^{A, B}(\mathbf{r}) & \rightarrow \tilde{D}_{q}^{A, B}(\mathbf{r}) e^{2 \mathrm{i} \varphi} \\
\tilde{C}_{q}^{A, B}(\mathbf{r}) & \rightarrow \tilde{C}_{q}^{A, B}(\mathbf{r}) e^{2 \mathrm{i} \varphi} \\
\tilde{D}_{q}^{A, B \sigma}(\mathbf{r}) & \rightarrow \tilde{D}_{q}^{A, B \sigma}(\mathbf{r}) e^{2 \mathrm{i} \varphi}, \\
\tilde{C}_{q}^{A, B \sigma}(\mathbf{r}) & \rightarrow \tilde{C}_{q}^{A, B \sigma}(\mathbf{r}) e^{2 \mathrm{i} \varphi},
\end{aligned}
$$

which is a special case of the transformation rules of pair densities under more general local gauge transformations discussed in Ref. [44], $\phi(\mathbf{r}) \rightarrow \varphi$. As repeatedly mentioned, the ingredients of the pair densities transform as two-body wave functions, so the pair densities transform with the square of the phase factor caught by each single-particle state. By contrast, one can easily show that all normal densities are invariant under global gauge transformations (but not necessarily local gauge transformations [21, 24, 44, 52, 55]), which simply follows from Eq. (E5) and originates in the hermiticity of the normal density matrices: the phase factors of a singleparticle state and its hermitian conjugate always cancel.
The same problem of clearly separating the parts of the local pair densities that have different symmetries also emerges when projecting symmetry-restricted HFB states on particle number, in which case only one of the two HFB states is globally gauge rotated [57, 85]. Again, the weights that multiply $\tilde{\varrho}_{j k}(\mathbf{r}, \mathbf{r})$ and $\tilde{\varsigma}_{j k, \mu}(\mathbf{r}, \mathbf{r})$ when summing up the local mixed pair densities become complex.

Another similarity transformation that constrains the possible combinations of terms in the EDF is Galilean invariance [21, 24, 52, 55], see also Appendix B. From the transformation of the pair density matrix under a Galilean boost transformation [4]

$$
\tilde{\rho}_{q}\left(\mathbf{r} \sigma, \mathbf{r}^{\prime} \sigma^{\prime}\right) \rightarrow \tilde{\rho}_{q}\left(\mathbf{r} \sigma, \mathbf{r}^{\prime} \sigma^{\prime}\right) e^{\mathrm{i} \mathbf{P} \cdot\left(\mathbf{r}+\mathbf{r}^{\prime}\right) / \hbar},
$$

where $\mathbf{P}$ is the vector of the center-of-mass momentum in the boosted frame, follows for example that

$$
\begin{aligned}
& \tilde{D}_{q}^{1,1}(\mathbf{r}) \rightarrow \tilde{D}_{q}^{1,1}(\mathbf{r}) e^{2 \mathbf{i} \mathbf{P} \cdot \mathbf{r} / \hbar}, \\
& \tilde{C}_{q}^{1,1}(\mathbf{r}) \rightarrow \tilde{C}_{q}^{1,1}(\mathbf{r}) e^{2 \mathrm{i} \mathbf{P} \cdot \mathbf{r} / \hbar},
\end{aligned}
$$

meaning that pair densities that are constructed to be real-valued functions in the rest frame necessarily become complex-valued functions in the boosted frame. This behavior is again very different from the one of normal densities, that remain real also in the boosted frame [44, 52, 55]. Pair densities containing gradients become complex in the boosted frame as well, but their transformation usually involves also other densities and/or gradients of other densities (as is the case for their normal counterparts), see Ref. [44] for a discussion in traditional notation. The same difference in behavior of normal and pair densities is also found for the arbitrary local gauge transformations considered in Refs. [44, 52, 55.

The relations above show that pair densities cannot be chosen to be real under all circumstances, but that there is an interest to separate the traditionally used expressions for pair densities into two different objects whose real and imaginary parts take the same symmetries in symmetry-restricted HFB calculations. This is achieved by the definitions (75)-(78). A pairing EDF that is constructed to be manifestly gaugeand Galilean-invariant is then necessarily composed of suitable bilinear forms of a pair density and the complex conjugate of a possibly different pair density as is the case for the pairing EDF of Eq. (148), such that the complex phase factors that arise from gauge or Galilean transformations cancel out. A more detailed analysis of Galilean invariance of particle-hole and possible particleparticle terms in the N2LO EDF using the conventions introduced here will be given elsewhere.

\section{Appendix F: Further comments on alternative densities at NLO}

The issues with alternative local densities, possible redundancies and reducibilities sketched in Sec. IIB 5 are 
much less of a problem for the standard NLO EDF than at higher orders. For the LO densities $\rho_{q}(\mathbf{r})=\boldsymbol{D}_{q}^{1,1}(\mathbf{r})$ and $\mathbf{s}_{q}(\mathbf{r})=D_{q}^{1,1 \sigma}(\mathbf{r})$ this is even trivially the case as they do not contain gradients. Containing just one gradient, because of Eqs. (87) and (89), neither of the two currents $\mathbf{j}_{q}(\mathbf{r})=\mathbf{C}_{q}^{1, \nabla}(\mathbf{r})$ and $J_{q, \mu \nu}(\mathbf{r})=C_{q, \mu \nu}^{1, \nabla \sigma}(\mathbf{r})$ that are customarily used to express the NLO EDF can be re-expressed through a different current of reduced to the gradient of some other current.

From a computational point of view, it is in general advantageous to construct the EDF from densities that can also be used to calculate frequently-used one-body observables. In this way, the number of densities that need to be constructed and stored can be kept as small as possible. Doing so gives a natural preference for the currents $\mathbf{j}_{q}(\mathbf{r})$ and $J_{q, \mu \nu}(\mathbf{r})$, which together with $\rho_{q}(\mathbf{r})$ and $\mathbf{s}_{q}(\mathbf{r})$ enter the expectation values of angular momenta, electromagnetic observables and the expectation values of the operators that are frequently used as constraints in the HFB equation. The same four local densities enter also the continuity equations for the transport of matter with given spin and isospin [56]. The only ambiguity in their definition concerns constant prefactors, where the choice provided by Eqs. (29), (30), (34), and (37) is the most symmetric one.

For the kinetic densities $\tau_{q}(\mathbf{r})=D_{q}^{(\nabla, \nabla)}(\mathbf{r}), \mathbf{T}_{q}(\mathbf{r})=$ $D_{q}^{(\nabla, \nabla) \sigma}(\mathbf{r})$, and $\mathbf{F}_{q}(\mathbf{r})=D_{q}^{\nabla,(\nabla \sigma)}(\mathbf{r})$ entering the NLO EDF, however, the situation is less straightforward. While $\tau_{q}(\mathbf{r})=D_{q}^{(\nabla, \nabla)}(\mathbf{r})$ as defined in Eq. (33) can be used without ambiguities to evaluate the one-body operator of kinetic energy

$$
\begin{aligned}
E_{\mathrm{kin}} & =\sum_{q=p, n} \frac{\hbar^{2}}{2 m_{q}} \int d^{3} r \sum_{j k} \rho_{k j}\left[\nabla \Psi_{j}^{\dagger}(\mathbf{r})\right] \cdot\left[\nabla \Psi_{k}(\mathbf{r})\right] \\
& =\sum_{q=p, n} \int d^{3} r \frac{\hbar^{2}}{2 m_{q}} D_{q}^{(\nabla, \nabla)}(\mathbf{r}),
\end{aligned}
$$

this definition of kinetic energy is not unique. An alternative form is given by

$$
E_{\mathrm{kin}}=-\sum_{q=p, n} \frac{\hbar^{2}}{2 m_{q}} \int d^{3} r \sum_{j k} \rho_{k j}
$$

$$
\begin{aligned}
& \times \frac{1}{2}\left\{\Psi_{j}^{\dagger}(\mathbf{r})\left[\Delta \Psi_{k}(\mathbf{r})\right]+\left[\Delta \Psi_{j}^{\dagger}(\mathbf{r})\right] \Psi_{k}(\mathbf{r})\right\} \\
= & \sum_{q=p, n} \int d^{3} r\left[-\frac{\hbar^{2}}{2 m_{q}} D_{q}^{1, \Delta}(\mathbf{r})\right],
\end{aligned}
$$

which is also frequently used in a non-hermitian form without the symmetrization in the first line on the righthand side of this equation. All these expressions give the same value for the kinetic energy.

While the calculation of $D_{q}^{1, \Delta}(\mathbf{r})$ and $\mathbf{D}_{q}^{1, \Delta \sigma}(\mathbf{r})$ only requires the storage and application of the Laplacian on the single-particle wave functions, which is less costly than the application and storage of the three components of the nabla, there are several reasons in favor of using $D_{q}^{(\nabla, \nabla)}(\mathbf{r})$ instead of $D_{q}^{1, \Delta}(\mathbf{r})$ and using $\mathbf{D}_{q}^{(\nabla, \nabla) \sigma}(\mathbf{r})$ instead of $\mathbf{D}_{q}^{1, \Delta \sigma}(\mathbf{r})$. On the one hand, the firstorder derivatives of the single-particle wave functions are required anyway to construct other densities and currents needed at NLO that cannot be re-expressed solely through Laplacians. On the other hand, as argued in Secs. IIID 2 and IV C, there are computational reasons to privilege densities for which the operators $\hat{A}$ and $\hat{B}$ are as balanced as possible.

The kinetic energy densities $\mathcal{E}_{\text {kin }}(\mathbf{r})$, which are provided in each case by the integrand in the second line of Eqs. (F1) and (F2), respectively, are however different because of 91 94]

$$
D_{q}^{(\nabla, \nabla)}(\mathbf{r})=-D_{q}^{1, \Delta}(\mathbf{r})+\frac{1}{2}\left[\Delta D_{q}^{1,1}(\mathbf{r})\right],
$$

which can be easily shown applying twice Eq. (101) and using the symmetry $D_{q}^{A, B}(\mathbf{r})=D_{q}^{B, A}(\mathbf{r})$, Eq. (87). Note that Refs. 91 95] work with an object that equals $-D_{q}^{1, \Delta}(\mathbf{r})$, and for which some authors [95] actually use the symbol $\tau_{q}(\mathbf{r})$. As demonstrated in these references, there are subtle differences when using one or the other expression for the kinetic energy as the starting point for semi-classical approximations to the kinetic energy density, such as gradient corrections in extended Thomas-Fermi methods. As has been argued in Refs. 93, 95], it is the average of the two different kinetic energy densities $\frac{1}{2}\left[D_{q}^{(\nabla, \nabla)}(\mathbf{r})-D_{q}^{1, \Delta}(\mathbf{r})\right]$ that establishes the connection to the average classical kinetic energy density, and not the kinetic density $D_{q}^{(\nabla, \nabla)}(\mathbf{r})$ that is traditionally used with the Skyrme EDF. After integration, both give of course the same value for the kinetic energy.
[1] M. Bender, P.-H. Heenen, and P.-G. Reinhard, Selfconsistent mean-field models for nuclear structure, Rev. Mod. Phys. 75, 121, (2003).

[2] M. Bender, N. Schunck, J. P. Ebran, and T. Duguet, Single-Reference and Multi-Reference Formulation, Chapter 3 of Energy Density Functional Methods for Atomic Nuclei, edited by N. Schunck (IOP, Bristol 2019), pp 3-1 to 3-78.
[3] S. Goriely, N. Chamel, and J. M. Pearson, HartreeFock-Bogoliubov nuclear mass model with $0.50 \mathrm{MeV}$ accuracy based on standard forms of Skyrme and pairing functionals. Phys. Rev. C 88, 061302(R) (2013).

[4] L. Bonneau, P. Quentin, and P. Möller, Global microscopic calculations of ground-state spins and parities for odd-mass nuclei. Phys. Rev. C 76, 024320 (2007). 
[5] J. Dobaczewski, A. V. Afanasjev, M. Bender, L. M. Robledo, and Y. Shi, Properties of nuclei in the nobelium region studied within the covariant, Skyrme, and Gogny energy density functionals., Nucl. Phys. A 944, 388 (2015).

[6] M. Kortelainen, T. Lesinski, J. Moré, W. Nazarewicz, J. Sarich, N. Schunck, M. Stoitsov, and S. Wild, Nuclear energy density optimization, Phys. Rev. C 82, 024313 (2010).

[7] M. Kortelainen, J. McDonnell, W. Nazarewicz, P.-G. Reinhard, J. Sarich, N. Schunck, M. Stoitsov, and S. Wild, Nuclear energy density optimization: Large deformations, Phys. Rev. C 85, 024304 (2012).

[8] M. Kortelainen, J. McDonnell, W. Nazarewicz, E. Olsen, P.-G. Reinhard, J. Sarich, N. Schunck, and S. Wild Nuclear energy density optimization: Shell structure, Phys. Rev. C 89, 054314 (2014).

[9] T. Lesinski, M. Bender, K. Bennaceur, T. Duguet, and J. Meyer, Tensor part of the Skyrme energy density functional: Spherical nuclei, Phys. Rev. C 76, 014312 (2007).

[10] M. Bender, K. Bennaceur, T. Duguet, P.-H. Heenen, T. Lesinski, and J. Meyer, Tensor part of the Skyrme energy density functional. II. Deformation properties of magic and semi-magic nuclei, Phys. Rev. C 80, 064302 (2009).

[11] V. Hellemans, P.-H. Heenen, and M. Bender, Tensor part of the Skyrme energy density functional. III. Time-odd parts at high spin, Phys. Rev. C 85, 014326 (2012).

[12] G. Colò, H. Sagawa, S. Fracasso, and P. F. Bortignon, Spin-orbit splitting and the tensor component of the Skyrme interaction, year:2010 Phys. Lett. B 646, 227 (2007).

[13] M. Zalewski, J. Dobaczewski, W. Satuła, and T. R. Werner, Spin-orbit and tensor mean-field effects on spinorbit splitting including self-consistent core polarizations, Phys. Rev. C 77, 024316 (2008).

[14] T. Lesinski, K. Bennaceur, T. Duguet, and J. Meyer, Isovector splitting of nucleon effective masses, ab initio benchmarks and extended stability criteria for Skyrme energy functionals, Phys. Rev. C 74, 044315 (2006).

[15] J. Erler, P. Klüpfel, and P.-G. Reinhard, Exploration of a modified density dependence in the Skyrme functional, Phys. Rev. C 82, 044307 (2010).

[16] S. Krewald, V. Klemt, J. Speth, and A. Faessler, Limits on the use of Skyrme forces, Nucl. Phys. A 281, 166 (1977).

[17] J. M. Pearson and M. Farine, Relativistic mean field theory and a density dependent spin orbit Skyrme force, Phys. Rev. C 50, 185 (1994).

[18] N. Chamel, S. Goriely, and J. M. Pearson, Further explorations of Skyrme-Hartree-Fock-Bogoliubov mass formulas. XI. Stabilizing neutron stars against a ferromagnetic collapse, Phys. Rev. C 80, 065804 (2009).

[19] J. Sadoudi, T. Duguet, J. Meyer, and M. Bender, Skyrme functional from a three-body pseudo-potential of second order in gradients. Formalism for central terms, Phys. Rev. C 88, 064326 (2013).

[20] J. Sadoudi, M. Bender, K. Bennaceur, D. Davesne, R. Jodon, and T. Duguet, Skyrme pseudo-potentialbased EDF parametrization for spuriosity-free MR-EDF calculations, Phys. Scr. T154, 014013 (2013).

[21] B. G. Carlsson, J. Dobaczewski, and M. Kortelainen, Local nuclear energy density functional at next-to-nextto-next-to-leading order, Phys. Rev. C 78, 044326 (2008).
[22] B. G. Carlsson, J. Dobaczewski, J. Toivanen and P. Veselý, Solution of self-consistent equations for the N3LO nuclear energy density functional in spherical symmetry. The program hosphe (v1.02). Comp. Phys. Comm. 181, 1641-1657 (2010).

[23] B. G. Carlsson and J. Dobaczewski, Convergence of Density-Matrix Expansions for Nuclear Interactions, Phys. Rev. Lett. 105, 122501 (2010).

[24] F. Raimondi, B. G. Carlsson, and J. Dobaczewski, Effective pseudopotential for energy density functionals with higher-order derivatives, Phys. Rev. C 83, 054311 (2011).

[25] T. H. R. Skyrme, Philos. Mag. 1, 1043 (1956).

[26] J. S. Bell and T. H. R. Skyrme, Philos. Mag. 1, 1055 (1956).

[27] T. H. R. Skyrme, Nucl. Phys. 9, 615 (1958/59).

[28] T. H. R. Skyrme, Nucl. Phys. 9, 635 (1958/59).

[29] D. Davesne, A. Pastore, and J. Navarro, Skyrme effective pseudopotential up to the next-to-next-to-leading order, J. Phys. G 40, 095104 (2013).

[30] D. Davesne, A. Pastore, and J. Navarro, Fitting N3LO pseudo-potentials through central plus tensor Landau parameters, J. Phys. G 41, 065104 (2014).

[31] D. Davesne, J. Navarro, P. Becker, R. Jodon, J. Meyer, and A. Pastore, Extended Skyrme pseudopotential deduced from infinite nuclear matter properties, Phys. Rev. C 91, 064303 (2015).

[32] D. Davesne, J. Meyer, A. Pastore, and J. Navarro, Partial wave decomposition of the N3LO equation of state, Phys. Scr. 90, 114002 (2015).

[33] D. Davesne, P. Becker, A. Pastore, and J. Navarro, Infinite matter properties and zero-range limit of nonrelativistic finite-range interactions, Ann. Phys. 375, 288 (2016).

[34] P. Becker, D. Davesne, J. Meyer, J. Navarro, and A. Pastore, Tools for incorporating a D-wave contribution in Skyrme energy density functionals, J. Phys. G 42, 034001 (2015).

[35] P. Becker, Développement d'une interaction nucléaire effective de nouvelle génération, $\mathrm{PhD}$ Thesis, Université Claude Bernard Lyon 1 (2017).

[36] P. Becker, D. Davesne, J. Meyer, J. Navarro, and A. Pastore, Solution of Hartree-Fock-Bogoliubov equations and fitting procedure using the N2LO Skyrme pseudopotential in spherical symmetry, Phys. Rev. C 96, 044330 (2017).

[37] P. Becker, A. Pastore, D. Davesne, and J. Navarro, Error analysis of the parameters of the Skyrme N2LO pseudopotential, Il Nuovo Cimento 42 C, 88 (2019).

[38] E. Chabanat, P. Bonche, P. Haensel, J. Meyer, and R. Schaeffer, A Skyrme parametrization from subnuclear to neutron star densities, Nucl. Phys. A 627, 710 (1997).

[39] E. Chabanat, P. Bonche, P. Haensel, J. Meyer, and R. Schaeffer, A Skyrme parametrization from subnuclear to neutron star densities Part II. Nuclei far from stabilities, Nucl. Phys. A 635, 231 (1998); Erratum Nucl. Phys. A 643, 441 (1998).

[40] A. Pastore, D. Davesne, K. Bennaceur, J. Meyer, and V. Hellemans, Fitting Skyrme functionals using linear response theory, Physica Scripta T154, 014014 (2013).

[41] W. Ryssens, Symmetry breaking in nuclear mean-field models, PhD Thesis, Université Libre de Bruxelles (2016). 
[42] W. Ryssens, M. Bender, and P.-H. Heenen, MOCCa code (unpublished).

[43] A. Pastore, D. Tarpanov, D. Davesne, and J. Navarro, Spurious finite-size instabilities in nuclear energy density functionals: Spin channel, Phys. Rev. C 92, 024305 (2015).

[44] E. Perlińska, S. G. Rohoziński, J. Dobaczewski, and W. Nazarewicz, Local density approximation for protonneutron pairing correlations: Formalism, Phys. Rev. C 69, 014316 (2004).

[45] W. Ryssens, V. Hellemans, M. Bender, and P.-H. Heenen, Solution of the Skyrme-HF+BCS equation on a 3D mesh, II: a new version of the EV8 code, Comp. Phys. Comm. 187, 175 (2015).

[46] W. Ryssens, M. Bender, and P.-H. Heenen, Iterative approaches to the self-consistent nuclear energy density functional problem. Heavy ball dynamics and potential preconditioning, Eur. Phys. J. A 55, 93 (2019).

[47] J. Dobaczewski, H. Flocard, and J. Treiner, HartreeFock-Bogolyubov description of nuclei near the neutrondrip line, Nucl. Phys. A 422, 103 (1984).

[48] J. Dobaczewski, J. Dudek, S. G. Rohoziński, and T. R. Werner, Point symmetries in the Hartree-Fock approach. I. Densities, shapes and currents, Phys. Rev. C 62, 014310 (2000).

[49] J. Dobaczewski, W. Nazarewicz, T. R. Werner, J. F. Berger, C. R. Chinn, and J. Dechargé, Mean-field description of ground-state properties of drip-line nuclei: Pairing and continuum effects, Phys. Rev. C 53, 2809 (1996).

[50] V. Rotival, Fonctionnelles d'énergie non-empiriques pour la structure nucléaire, $\mathrm{PhD}$ Thesis, Université Paris Diderot (2009).

[51] S. G. Rohoziński, J. Dobaczewski, and W. Nazarewicz, Self-consistent symmetries in the proton-neutron Hartree-Fock-Bogoliubov approach, Phys. Rev. C 81, 014313 (2010).

[52] J. Dobaczewski and J. Dudek, Time-odd components in the rotating mean field and identical bands, Acta Phys. Pol. B 27, 45 (1996).

[53] K. Bennaceur and J. Meyer, discussion notes (unpublished).

[54] Y. M. Engel, D. M. Brink, K. Goeke, S. J. Krieger, and D. Vautherin, Time-dependent Hartree-Fock theory with Skyrme's interaction, Nucl. Phys. A 249, 215 (1975).

[55] J. Dobaczewski and J. Dudek, Time-odd components in the mean field of rotating superdeformed nuclei, Phys. Rev. C 52, 1827 (1995); Erratum ibid 55, 3177 (1997).

[56] F. Raimondi, B. G. Carlsson, J. Dobaczewski, and J. Toivanen, Continuity equations and local gauge invariance for the N3LO nuclear energy density functionals, Phys. Rev. C 84, 064303 (2011).

[57] M. Bender, T. Duguet, and D. Lacroix, Particlenumber restoration within the energy density functional formalism Phys. Rev. C 79, 044319 (2009).

[58] S. Stringari and D. M. Brink, Constraints on effective interactions imposed by antisymmetry and charge independence, Nucl. Phys. A 304, 307 (1978).

[59] J. Terasaki, P.-H. Heenen, P. Bonche, J. Dobaczewski, and H. Flocard, Superdeformed rotational bands with density dependent pairing interactions, Nucl. Phys. A 593, 1 (1995).

[60] C. Rigollet, P. Bonche, H. Flocard, and P.-H. Heenen, Microscopic study of the properties of identical bands in the $A=150$ mass region, Phys. Rev. C 59, 3120 (1999).

[61] B. Gall, P. Bonche, J. Dobaczewski, H. Flocard, and P.-H. Heenen, Superdeformed rotational bands in the mercury region. A cranked Skyrme-Hartree-FockBogoliubov study, Z. Phys. A 348, 183 (1994).

[62] D. Baye and P.-H. Heenen, Generalised meshes for quantum mechanical problems, J. Phys. A 19, 2041 (1986).

[63] D. Baye, The Lagrange-mesh method, Phys. Rep. 565, 1 (2015).

[64] W. Ryssens, P.-H. Heenen, and M. Bender, Numerical accuracy of mean-field calculations in coordinate space, Phys. Rev. C 92, 064318 (2015).

[65] R. Jodon, M. Bender, K. Bennaceur, and J. Meyer, Constraining the surface properties of effective Skyrme interactions, Phys. Rev. C 94, 024335 (2016).

[66] W. Ryssens, M. Bender, K. Bennaceur, P.-H. Heenen, and J. Meyer, The impact of the surface energy coefficient on the deformation properties of atomic nuclei as predicted by Skyrme energy density functionals, Phys. Rev. C 99, 044315 (2019).

[67] N. Schunck, J. Dobaczewski, J. McDonnell, J. Moré, W. Nazarewicz, J. Sarich, and M. V. Stoitsov, Onequasiparticle states in the nuclear energy density functional theory, Phys. Rev. C 81, 24316 (2010).

[68] S. Fracasso, E. B. Suckling, and P. D. Stevenson, Unrestricted Skyrme-tensor time-dependent HartreeFock model and its application to the nuclear response from spherical to triaxial nuclei, Phys. Rev. C 86, 044303 (2012).

[69] V. Hellemans, A. Pastore, T. Duguet, K. Bennaceur, D. Davesne, J. Meyer, M. Bender, and P.-H. Heenen, Spurious finite-size instabilities in nuclear energy density functionals, Phys. Rev. C 88, 064323 (2013).

[70] F. Becker et al., Coulomb excitation of ${ }^{78} \mathrm{Kr}$, Nucl. Phys. A 770, 107 (2006).

[71] E. Clément et al., Shape coexistence in neutron-deficient krypton isotopes, Phys. Rev. C 75, 054313 (2007).

[72] K. Wimmer et al., Shape coexistence revealed in the $N=$ $Z$ isotope ${ }^{72} \mathrm{Kr}$ through inelastic scattering, Eur. Phys. J. A 56, 159 (2020).

[73] J. Döring et al., High-spin bands in ${ }^{80} \mathrm{Kr}$, Phys. Rev. C 52, 76 (1995).

[74] M. Bender, P. Bonche, and P.-H. Heenen, Shape coexistence in neutron-deficient Kr isotopes: Constraints on the single-particle spectrum of self-consistent meanfield models from collective excitations, Phys. Rev. C 74, 024312 (2006).

[75] P. Bonche, H. Flocard, P.-H. Heenen, S. J. Krieger, and M. S. Weiss, Self-consistent study of triaxial deformations: Application to the isotopes of $\mathrm{Kr}, \mathrm{Sr}, \mathrm{Zr}$ and Mo, Nucl. Phys. A 443, 39 (1985).

[76] M. Girod, J. P. Delaroche, A. Görgen, and A. Obertelli, The role of triaxiality for the coexistence and evolution of shapes in light krypton isotopes, Phys. Lett. B 676, 39 (2009).

[77] T. R. Rodríguez, Structure of krypton isotopes calculated with symmetry-conserving configurationmixing methods, Phys. Rev. C 90, 034306 (2014).

[78] I. Angeli and K. P. Marinova, Table of experimental nuclear ground state charge radii: An update, At. Data Nucl. Data Tables 99, 69 (2013).

[79] S. Raman, C. W. Nestor, and P. Tikkanen, Transition probability from the ground to the first-excited $2^{+}$state 
of even-even nuclides, At. Data Nucl. Data Tables 78, 1 (2001).

[80] M. Bender, G. F. Bertsch, and P.-H. Heenen, Global study of quadrupole correlation effects, Phys. Rev. C 73, 034322 (2006).

[81] National Nuclear Data Center, information extracted from the NuDat 2 database, https:www.nndc.bnl.govnudat2

[82] C. Ling, C. Zhou, and Y. Shi, Fission barriers of actinide nuclei with nuclear density functional theory: influence of the triaxial deformation, Eur. Phys. J. A 56, 180 (2020).

[83] B. N. Lu, J. Zhao, E. G. Zhao, and S. G. Zhou, Multidimensionally-constrained relativistic mean-field models and potential-energy surfaces of actinide nuclei, Phys. Rev. C 89, 014323 (2014).

[84] B. Singh, R. Zywina, and R. B. Firestone, Table of superdeformed nuclear bands and fission isomers, Nucl. Data Sheets 97, 241 (2002).

[85] L. M. Robledo, Particle number restoration: Its implementation and impact in nuclear structure calculations, Int. J. Mod. Phys. E 16, 337 (2007).

[86] T. Duguet, M. Bender, K. Bennaceur, D. Lacroix, and T. Lesinski, Particle-number restoration within the energy density functional formalism: Non-vialibility of terms depending on non-integer powers of the density matrices, Phys. Rev. C 79, 044320 (2009).

[87] L. M. Robledo, Remarks on the use of projected densities in the density-dependent part of Skyrme or Gogny functionals, J. Phys. G 37, 064020 (2010).

[88] J. Dobaczewski, J. Dudek, S. G. Rohoziński, and T. R. Werner, Point symmetries in the Hartree-Fock approach. II. Symmetry-breaking schemes, Phys. Rev. C 62, 014311 (2000).

[89] P. Bonche, H. Flocard, and P.-H. Heenen, Self-consistent calculation of nuclear rotations: The complete yrast line of ${ }^{24} \mathrm{Mg}$, Nucl. Phys. A 467, 115 (1987).

[90] B. Bally and M. Bender, Projection on particle number and angular momentum: Example of triaxial Bogoliubov quasiparticle states, Phys. Rev. C 103024315 (2021).

[91] C. Y. Wong, On the Thomas-Fermi approximation of the kinetic energy density, Phys. Lett. B 63, 395 (1976).

[92] H. Gräf, Thomas-Fermi kinetic-energy density with gradient corrections, Nucl. Phys. A 343, 91 (1980).

[93] R. J. Lombard, D. Mas, and S. A. Moszkowski, On the kinetic energy density, J. Phys. G 17, 455 (1991).

[94] R. J. Lombard and S. A. Moszkowski, On the positivity of the kinetic-energy density, Il Nuovo Cim. 109, 1291 (1994).

[95] M. Prakash, S. Shlomo, and V. M. Kolomietz, Shell and surface effects in the static Wigner phase-space distribution function of nuclei, Nucl. Phys. A 370, 30 (1981). 